MARISTELLA SVAMPA

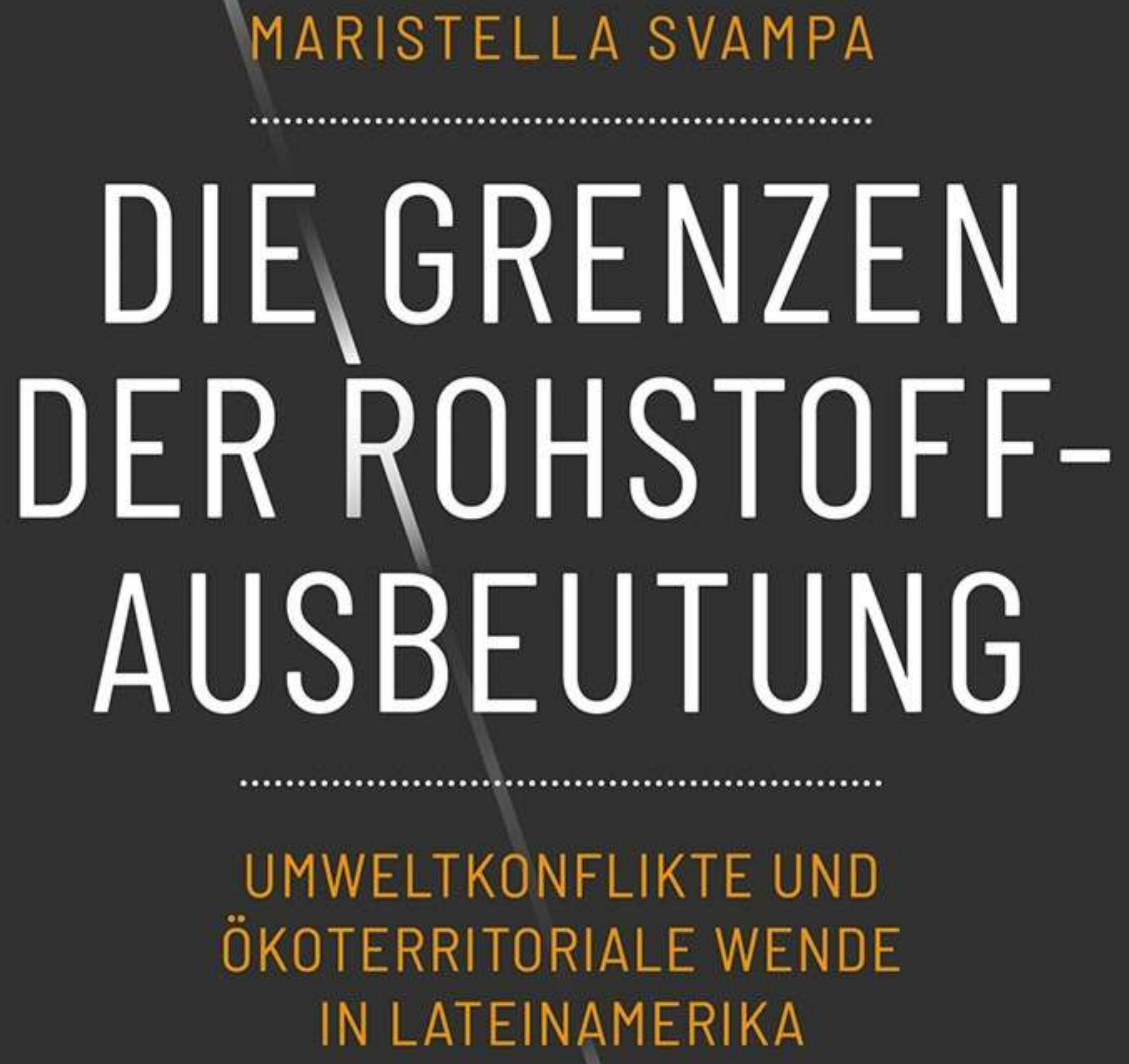

BIELEFELD UNIVERSITY PRESS 
Maristella Svampa

Die Grenzen der Rohstoffausbeutung

BiUP General 
Maristella Svampa ist Soziologin, Schriftstellerin und Forscherin. Sie hat an der Nationalen Universität Córdoba Philosophie studiert und an der École des Hautes Études en Sciences Sociales in Paris in Soziologie promoviert. Sie ist Professorin für lateinamerikanische Gesellschaftstheorie an der Universidad Nacional de La Plata (Argentinien). Im Jahr 2006 erhielt sie das Guggenheim-Stipendium und das Konex-Diplom in Soziologie, 2014 das Konex-Diplom in politischem und soziologischem Essay und 2016 den Konex-Platinpreis in Soziologie. 2018 war sie Fellow des Maria Sibylla Merian Center for Advanced Latin American Studies (CALAS). 
Maristella Svampa

\section{Die Grenzen der Rohstoffausbeutung}

Umweltkonflikte und ökoterritoriale Wende in Lateinamerika

Übersetzt aus dem Spanischen von Ann-Kathrin Lauer und Lisa-Marie Maier

\section{BIELEFELD UNIVERSITY PRESS}






We acknowledge support for the publication costs by the Open Access Publication Fund of Bielefeld University.

\section{Bibliografische Information der Deutschen Nationalbibliothek}

Die Deutsche Nationalbibliothek verzeichnet diese Publikation in der Deutschen Nationalbibliografie; detaillierte bibliografische Daten sind im Internet über http://dnb.d-nb.de abrufbar.$$
\text { (c) }(1) \Theta
$$

Dieses Werk ist lizenziert unter der Creative Commons Attribution-NoDerivatives 4.0 Lizenz (BY-ND). Diese Lizenz erlaubt unter Voraussetzung der Namensnennung des Urhebers die Vervielfältigung und Verbreitung des Materials in jedem Format oder Medium für beliebige Zwecke, auch kommerziell, gestattet aber keine Bearbeitung. (Lizenztext: https://creativecommons.org/licenses/by-nd/4.o/deed.de)

Um Genehmigungen für Adaptionen, Übersetzungen oder Derivate einzuholen, wenden Sie sich bitte an rights@transcript-publishing.com

Die Bedingungen der Creative-Commons-Lizenz gelten nur für Originalmaterial. Die Wiederverwendung von Material aus anderen Quellen (gekennzeichnet mit Quellenangabe) wie z.B. Schaubilder, Abbildungen, Fotos und Textauszüge erfordert ggf. weitere Nutzungsgenehmigungen durch den jeweiligen Rechteinhaber.

\section{(C) 2020 Bielefeld University Press}

\section{An Imprint of transcript Verlag}

http://www.bielefeld-university-press.de

Umschlaggestaltung: Maria Arndt, Bielefeld

Lektorat: Martin Breuer, Olaf Kaltmeier

Übersetzung aus dem Spanischen: Ann-Kahrin Lauer und Lisa-Marie Maier

Druck: Majuskel Medienproduktion $\mathrm{GmbH}$, Wetzlar

Print-ISBN 978-3-8376-5378-6

PDF-ISBN 978-3-8394-5378-0

https://doi.org/10.14361/9783839453780

Gedruckt auf alterungsbeständigem Papier mit chlorfrei gebleichtem Zellstoff. Besuchen Sie uns im Internet: https://www.transcript-verlag.de Unsere aktuelle Vorschau finden Sie unter www.transcript-verlag.de/vorschau-download 


\section{Inhalt}

Einleitung ................................................... 7

Kapitel 1: Neo-Extraktivismus und Entwicklung ..................... 11

Extraktivismus und $\mathrm{NeO}$-Extraktivismus .......................... 11

Der Neo-Extraktivismus als »privilegiertes Fenster « .................... 15

Neo-Extraktivismus als Entwicklungspfad und sozio-territoriales Modell ....... 19

»Rohstoff-Konsens« und Entwicklungsillusionen ...................... 22

Kapitel 2: Sozio-ökologische Konflikte und Sprachen der Inwertsetzung ... 31

Phasen des Neo-Extraktivismus .................................. 31

Territorialität und eine neue Sprache der Inwertsetzung ............... 39

Politisch-ideologische Matrizen und ökoterritoriale Wende der Konflikte ..... 42

Sozio-ökologische Konflikte und ihre Ebenen ....................... 47

Kapitel 3: Reichweite der ökoterritorialen Wende .................. 57

Aspekte der ökoterritorialen Wende ............................ 57

Neo-Extraktivismus und indigene Gruppen .......................... 61

Populare Feminismen des Südens ............................ 69

Kapitel 4: Auf dem Weg zu einem Neo-Extraktivismus der Extremfiguren .. 75

Der Vormarsch extraktivistischer Gewalt ........................... 75

Enklaven und kriminelle Territorialitäten ......................... 78

Das andere Gesicht des Patriarchats: Extraktivismus und Gewaltketten ...... 83

Die Expansion der Extremenergien und neue Konflikte ................ 86

Die Erweiterung der Geographie der Ressourcengewinnung ............. 93 
Kapitel 5: Ende des progressiven Zyklus und neue Abhängigkeiten ...... 99

China und der Rahmen der neuen Unabhängigkeit.................... 99

Das Ende des progressiven Zyklus als lingua franca ...................... 105

Die Grenzen des real existierenden Progressismus .................... 110

Abschließende Überlegungen: Die Dimensionen der Systemkrise ........ 115

Dimensionen der Krise: Das Anthropozän .......................... 116

Anthropozän, Kritik des Neo-Extraktivismus und Alternativen .............. 121

Gaia und die Wege der Interdependenz ............................ 127

Die Dimensionen der Krise in Lateinamerika ........................ 132

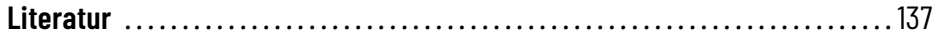




\section{Einleitung}

$\mathrm{Zu}$ Beginn des 21. Jahrhunderts profitierten die lateinamerikanischen Volkswirtschaften von den hohen Weltmarktpreisen für Rohstoffe (commodities) und es begann eine neue Ära des Wirtschaftswachstums. Diese neue Konjunktur fiel mit einem politischen Epochenwechsel zusammen, der vor allem durch die Kritik des sogenannten Washington Consensus aus den 1980er Jahren, starken sozialen Mobilisierungen und die Infragestellung traditioneller Formen politischer Repräsentation gekennzeichnet war. Dieser Protestzyklus gipfelte in mehreren Ländern der Region in der Entstehung progressiver Links- oder Mitte-LinksRegierungen, die - ungeachtet ihrer Unterschiede - eine heterodoxe Wirtschaftspolitik verfolgten, indem sie die Sozialausgaben erhöhten und gleichzeitig soziale Integration durch eine Steigerung des Konsums förderten. Damit begann in Lateinamerika der so genannte progressive Zyklus, der mindestens bis in die Jahre 2015/2016 hinein andauerte.

In dieser wirtschaftlich äußert profitablen Phase tendierten die lateinamerikanischen Regierungen unabhängig von ihrer ideologischen Ausrichtung dazu, voll auf die komparativen Kostenvorteile des Rohstoffbooms zu setzen und dabei die neuen wirtschaftlichen, sozialen, ökologischen und territorialen Ungleichgewichte zu verharmlosen, die der massive Export von Rohstoffen mit sich brachte. Im Laufe der Jahre haben ausnahmslos alle lateinamerikanischen Regierungen die Rückkehr einer produktivitätsorientierten Entwicklungsvision ermöglicht, die kritische Diskussionen über die Folgen des extraktivistischen Exportmodells erschwert oder gar unterbindet. Darüber hinaus setzten 
die Regierungen bewusst auf die Implementierung großer Bergbauund Staudammprojekte und erweiterten gleichzeitig die Erdöl- und Agrargrenze, letztere v.a. mit dem Anbau von Monokulturen wie Sojabohnen, Biokraftstoffen und Ölpalmen.

Mit dem Aufkommen vielfältiger sozialer Konflikte bildete sich im Verlauf der Zeit ein Konzept heraus, mit dem sich die beschriebene Entwicklung analytisch fassen lässt und dem das Potential zur sozialen Mobilisierung innewohnt: Neo-Extraktivismus. Es ist klar, dass es sich hierbei nicht um ein ganz neues Phänomen handelt, da die Ursprünge des Extraktivismus zweifellos auf die europäische Eroberung und Kolonisierung Lateinamerikas und den damit einsetzenden Beginn des Kapitalismus zurückzuführen sind. Allerdings erhielt das Phänomen des Extraktivismus in der Hitze des jungen 21. Jahrhunderts eine neue Dimension, nicht nur in objektiver Hinsicht - wegen der Anzahl und des Ausmaßes extraktivistischer Projekte und Aktivitäten sowie der beteiligten nationalen und transnationalen Akteure -, sondern auch in subjektiver Hinsicht - aufgrund des Entstehens großer sozialer Widerstände, die den schwindelerregenden Vorstoß der Rohstoffgrenze in Frage stellten, und angesichts der Konflikte um die Verteidigung alternativer Werte - Boden, Territorien, Gemeinschaftsgüter, Natur -, in deren Rahmen allmählich alternative Sichtweisen und Narrative ausgearbeitet wurden.

Zugleich führte die emergente Konfliktsituation, die durch die neue Dynamik der Kapitalakkumulation und der damit verbundenen Bedrohung von Naturgütern, Ländereien und Territorien ausgelöst wurde, $\mathrm{zu}$ massiven Konfrontationen zwischen kleinbäuerlich-indigenen Organisationen, sozio-territorialen Bewegungen, Umweltkollektiven einerseits und Regierungen und großen Wirtschaftsunternehmen andererseits. $\mathrm{Zu}$ letzteren gehörten nicht nur konservative und neoliberale Regime, sondern auch jene progressiven Regierungen, die so viele politische Erwartungen geweckt hatten. Die neue Phase, die bereits als Neo-Extraktivismus definiert worden war, führte zu Dilemmata und Brüchen in der Organisation sozialer Bewegungen sowie innerhalb der Linken, die die Grenzen des real-existierenden Progressivismus aufzeigten. Diese Grenzen wurden in ihrer Verbindung mit autoritä- 
ren politischen Praktiken und hegemonialen Entwicklungsphantasien sichtbar. Mit dem Ende des so genannten "Rohstoff Superzyklus« um das Jahr 2013 sehen wir uns nun mit einer Intensivierung des Neo-Extraktivismus in allen Ländern konfrontiert. Die Konsolidierung der Gleichung »mehr Extraktivismus, weniger Demokratie« (Svampa 2016) wird aktuell anhand der Flexibilisierung der ohnehin nur spärlich durchgeführten Umweltkontrollen sowie einer sich weiter verschärfenden Kriminalisierung von und die zunehmenden Morde an UmweltaktivistenInnen im Kontext des Kampfes um Zugang zu Land und Naturgütern deutlich.

Im vorliegenden Text schlage ich eine programmatische Synthese vor, die die Expansion des Neo-Extraktivismus in der lateinamerikanischen Region in fünf Kapiteln aufzeigt. In diesem Sinne werde ich mich im ersten Kapitel darauf konzentrieren, einige kritische Konzepte, die die Debatte prägen, vorzustellen. Dazu gehören Neo-Extraktivismus, Rohstoffkonsens und Entwicklungsillusion. Um ihre Relevanz zu begründen, werde ich erläutern, wie diese Konzepte die aktuelle Krise in ihren verschiedenen Dimensionen beleuchten. Im zweiten Kapitel werde ich mich mit dem sozio-ökologischen Konflikt, seinen verschiedenen Dimensionen sowie der neuen Modalität der Bewertung von umkämpften Territorien, die ich als ökoterritoriale Wende bezeichnet habe, auseinandersetzen. Im dritten Kapitel widme ich mich den vielschichtigen Entwicklungen rund um die ökoterritoriale Wende, welche die sozio-ökologischen Konflikte derzeit auszeichnet, und arbeite die Dilemmata heraus, mit denen die Anerkennung indigener Rechte sowie die Ausweitung neuer Formen des popularen Feminismus in der Region verbunden sind. In Kapitel vier werde ich auf die neue Phase des NeoExtraktivismus in seiner extremsten Ausprägung eingehen: kriminelle Territorialitäten, staatliche und halbstaatliche Gewalt, patriarchalische Gewalt, kurzgefasst: die Expansion extremer Kräfte. Im fünften Kapitel werden der geopolitische Kontext und die Formen, die die neue Abhängigkeit von China annimmt, untersucht. Ebenso werden die Grenzen des progressiven Zyklus untersucht und eine generelle Bilanz, die über die Expansion des Neo-Extraktivismus hinausgeht, gezogen. 
Letztlich schließt das Buch mit einer Reflexion über die systembedingte Krise ab. Hierzu wird das Konzept des Anthropozäns aufgegriffen. Letzteres umfasst eine Diagnose, die die sozial-ökologische Krise planetarischen Umfangs mit der Kritik an aktuellen Entwicklungsmodellen verbindet. Gleichzeitig werden einige der horizontalen Konzepte weiterentwickelt, die in den kritischen Analysen und den Sprachen antihegemonialer sozialer Bewegungen sowohl in Lateinamerika als auch in Europa präsent sind. 


\section{Kapitel 1: Neo-Extraktivismus und Entwicklung}

In diesem ersten Kapitel stelle ich die grundlegenden kritischen Konzepte vor, die der Analyse in diesem Buch zugrunde liegen, das heißt den Neo-Extraktivismus, den Rohstoffkonsens und die Entwicklungsillusion. Zudem zeige ich die Brüche und Kontinuitäten zwischen Extraktivismus und Neo-Extraktivismus auf.

\section{Extraktivismus und Neo-Extraktivismus}

Der in Lateinamerika entstandene Begriff des Neo-Extraktivismus ist eine sehr aufschlussreiche analytische Kategorie, die sowohl hohes deskriptives und erklärendes Potential aufweist als auch durch ihren anprangernden Charakter eine starke mobilisierende Wirkung hat. Der Begriff wird sowohl als analytische Kategorie als auch als stark politisch geprägtes Konzept verwendet. Er bezieht sich also sehr deutlich auf Machtverhältnisse und aktuelle Konflikte und verweist über die tatsächlich existierenden Asymmetrien hinaus auf einen Komplex geteilter Verantwortung, der sowohl den Globalen Norden als auch den Globalen Süden, Zentrum und Peripherie gleichermaßen betrifft. Des Weiteren zeigt die Kategorie auf komplexe Problematiken, welche die aktuelle multiple Krise auszeichnen, indem sie auf das unhaltbare derzeitige Entwicklungsmodell hinweist und vor einer fortschreitenden Logik der sozialen Enteignung (desposesión) warnt.

Aufgrund der Fülle von Veröffentlichungen zur Thematik wäre es unmöglich, alle Beiträge und Facetten der Debatte hier zusammenzu- 
fassen, etwa auch was die Verwendung des Begriffes durch die betroffenen Akteure und sozialen Bewegungen betrifft. Zunächst sollen einige Veröffentlichungen, die die Mehrdimensionalität und Mehrstufigkeit des Phänomens unterstreichen, vorgestellt werden. So sind sich zum Beispiel alle Autoren hinsichtlich der historischen Wurzeln des "Akkumulationsmodells des Extraktivismus « einig. Für den ecuadorianischen Ökonom Alberto Acosta »ist der Extraktivismus ein Akkumulationsmodell, das sich vor 500 Jahren erheblich durchzusetzen begann« und das seitdem von der Nachfrage der Metropolen des emergenten Kapitalismus bestimmt wird (Acosta 2012). In diesem Sinne stellt der Argentinier Horacio Machado Aráoz fest, der Extraktivismus sei weder eine Phase des Kapitalismus noch ein Problem gewisser unterentwickelter Ökonomien, sondern stelle vielmehr ein "strukturelles Merkmal des Kapitalismus als weltweites Wirtschaftsmodell« und »historisch-geopolitisches Produkt der ursprünglichen Hierarchie zwischen Kolonialgebieten und imperialistischen Metropolen« dar, »bei denen eine Seite als bloßer Raum für Profit und Plünderung zwecks der Versorgung der anderen Seite wahrgenommen wird « (Machado Aráoz 2013). Damit konform argumentiert der Venezolaner Emiliano Terán Mantovani, dass der Neo-Extraktivismus als »Akkumulationsmodell« gelesen werden kann, insbesondere in Bezug auf die lateinamerikanischen Ökonomien, »die aus dem sozialen und territorialen, also den Nationalstaat umfassenden Wirkungsbereich, untersucht werden können, ohne dabei andere Ebenen der territorialen Analyse zu vernachlässigen « (Terán, 2016, S. 257).

Weitere wichtige Arbeiten untersuchen den Extraktivismus als Entwicklungsstrategie, die auf dem Abbau und der Aneignung der Natur basiert und »die als Versorger von Rohstoffen ein produktives, kaum diversifiziertes und stark von einer internationalen Eingliederung abhängiges Netzwerk versorgt«. Somit bedeutet der Extraktivismus für den Uruguayer Eduardo Gudynas eher eine "Art der Aneignung« statt eine Art der Produktion; diese sei »eine Form des Abbaus der natürlichen Ressourcen«, womit Tätigkeiten zum Abbau natürlicher Ressourcen in großen Mengen oder mit hoher Intensität gemeint sind, die nicht (oder begrenzt) verarbeitet werden und für den Export 
gedacht sind. Im Laufe der Geschichte haben sich aufeinanderfolgende Generationen des Extraktivismus gebildet, von denen aktuell die dritte und vierte Generation herausstechen, welche sich durch den intensiven Verbrauch von Wasser, Energie und Rohstoffen auszeichnen. Demnach herrschen Unterschiede zwischen dem traditionellen Extraktivismus - den die konservativen Regierungen der Region replizieren - und dem Neo-Extraktivismus, einem neuen Modell, bei dem der Staat eine aktivere Rolle bei der Abschöpfung und Verteilung der Gewinne einnimmt und so eine gewisse soziale Legitimierung erreicht, obwohl die negativen sozialen und ökologischen Folgen offensichtlich überwiegen (Gudynas 2009 und 2015).

Meiner Einschätzung nach und in Übereinstimmung mit den hier zitierten Analysen ist die historische und strukturelle Beschaffenheit des Extraktivismus an die »Erfindung Europas « und die Expansion des Kapitals gebunden. In diesem Sinne reicht der Extraktivismus bis zur conquista Lateinamerikas und dem Genozid der indigenen Völker zurück. Seither ist Lateinamerika eine von Zerstörung und Ausplünderung geprägte Region, die sich mit ihrem Ressourcenreichtum immer wieder an die sich verändernden Wirtschaftszyklen anpasste und von der Logik des Kapitals gesteuert wurde. Dies geschah durch die Expansion der Binnengrenzen, die sowohl auf lokaler Ebene starke Kontraste zwischen außergewöhnlicher Rentabilität und extremer Armut mit sich brachte als auch einen hohen Verlust an Menschenleben und die Degradation von ganzen Landstrichen nach sich zog, die so zu "geopferten Gebieten« wurden. Die Potosí im heutigen Bolivien markierte die Geburtsstunde der Kapitalakkumulation und der umfassenden Aneignung der Natur in Lateinamerika, die durch Rohstoffexport und die abhängige Integration in die Weltwirtschaft gekennzeichnet sind. Interne Spezialisierung und externe Abhängigkeit konsolidierten ein Modell, das der Venezolaner Fernando Coronil später treffend als »Naturexportierende Gesellschaften« beschrieb.

Die Geschichte des Extraktivismus in der Region war keineswegs geradlinig, sondern wurde von aufeinanderfolgenden Wirtschaftszyklen durchzogen. Diese wurden einerseits von der Nachfragesituation 
auf dem Weltmarkt bestimmt, ${ }^{1}$ andererseits aber auch durch Phasen der Konsolidierung des Nationalstaats geprägt (vor allem in der Mitte des 20. Jahrhunderts). Dabei ermöglichte die Festigung der Staatswesen eine gewisse Kontrolle der außergewöhnlichen Gewinne, die sowohl im Bergbau- als auch im Erdölsektor erwirtschaftet wurden.

$\mathrm{Zu}$ Beginn des 21. Jahrhunderts traten neue Dimensionen des Extraktivismus hervor. In der gegenwärtigen Situation, die sich durch Kontinuitäten und Brüche zum historischen Extraktivismus auszeichnet, erscheint das alte Konzept in neuer Form, als Neo-Extraktivismus. Man spricht von Kontinuitäten, weil in der Schärfe der aufeinanderfolgenden Wirtschaftszyklen die extraktivistische DNA, mit der das europäische Kapital das Langzeitgedächtnis der Region prägte, auch eine bestimmte soziale Vorstellung von der Natur und ihren Gütern geprägt hatte. Assoziiert wurde der Extraktivismus folglich nicht nur mit dem Raub und der Plünderung von Naturressourcen im großen Stil, sondern auch mit Wettbewerbsvorteilen und wirtschaftlichen Vorteilen, die sich nach den unterschiedlichen Wirtschaftszyklen und der Rolle des Staates richteten. Nicht zufällig hat der Neo-Extraktivismus angesichts des vorherrschenden Progressismus die Entwicklungsillusion wiederbelebt. Diese suggeriert, dass das Erreichen eines höheren Entwicklungsstandes über eine Ausweitung der Förderung von und des Handels mit Rohstoffen und über die daraus resultierenden wirtschaftlichen Vorteile, gerade bei einer aktiven Rolle des Staates, möglich sei.

Von Brüchen ist die Rede, weil die neue Phase der Kapitalakkumulation, die erheblichen Druck auf Naturgüter und Landstriche ausübt und noch stärker auf die rasante Ausweitung der Commodity-Grenzen zielt, neue Wege für politische, soziale und ökologische Diskussionen sowie für im Rahmen der Entwicklungsillusion zuvor ungesehenen sozialen Widerstand öffnet. Es kommt zu neuen Debatten und kollektiven schaulicht werden, die vom Silberzyklus in einen Boom für Kautschuk, Guano, Salpeter sprang und neuerdings einen Aufschwung im Bergbau erlebt. 
Aktionen, die die Entwicklungsillusion in Frage stellen und gleichzeitig die weitere Vertiefung eines Modells anprangern, das tendenziell die Förderung eines einzigen Produktes vorsieht, die Biodiversität zerstört sowie das Zurückhalten fruchtbaren Landes und die Zerstörung von ganzen Territorien mit sich bringt.

\section{Der Neo-Extraktivismus als "privilegiertes Fenster «}

Für das Verständnis des zeitgenössischen Neo-Extraktivismus schlage ich eine Interpretation auf zwei Ebenen vor: eine allgemeinere, die ihn als privilegiertes Fenster zur Darstellung der Dimensionen der aktuellen Krise darstellt, und eine spezifischere, die den Neo-Extraktivismus als ein soziopolitisch-territoriales Modell versteht, das auf nationaler, regionaler oder lokaler Ebene plausibel analysiert werden kann. Nach meinem Verständnis stellt der Neo-Extraktivismus, in seinen während der letzten 15 Jahre in Lateinamerika entstandenen Versionen ein komplexes Konzept dar. Er bildet auf verschiedenen Ebenen ein privilegiertes Fenster, um die vielfältigen Krisen zu lesen, die die zeitgenössischen Gesellschaften prägen.

Zuerst einmal steht der Neo-Extraktivismus im Mittelpunkt der zeitgenössischen Akkumulation. Tatsächlich, wie mehrere Autoren bereits dargelegt haben, erfordert die Zunahme des sozialen Metabolismus des Kapitals im Rahmen des fortgeschrittenen Kapitalismus immer mehr Rohstoffe und Energien für seine Erhaltung, was wiederum zu einem stärkeren Druck auf Naturressourcen und Territorien führt. Obwohl der metabolische Austausch zwischen Mensch und Natur ein Thema ist, das sich nur am Rande durch die Schriften von Karl Marx ${ }^{2}$ zieht, wurde es in jüngerer Zeit von mehreren Vertretern

2 Wie Michael Löwy (2011) in seinen Schriften betont, distanziert sich diese kritische Perspektive, die mit dem metabolischen Austausch zwischen Mensch und Natur verbunden ist (der die ökologische Krise auslöst), von der produktivistischen Seite des vorherrschenden Marxismus im 20. Jahrhundert. Mehr zum Thema, siehe Sacher (2016) und auch Delgado (2016). 
des kritischen (und ökologischen) Marxismus aufbereitet. Sowohl J. O'Connor (2001) als auch John Bellamy Foster (2000) heben die Kosten der Naturelemente hervor, die im konstanten und variablen Kapital, der Bodenrente und auch in negativen Externalitäten aller Art enthalten sind. Während Bellamy Foster von »der metabolischen Fraktur" spricht, nennt O'Connor diesen Prozess »den zweiten Widerspruch des Kapitalismus « und weist darauf hin, dass »es keinen Einzelbegriff gibt, der die gleiche theoretische Interpretation hat wie die Ausbeutungsrate im ersten Widerspruch (Kapital/Arbeit). Ebenso heben beide Autoren die Aneignung und selbstzerstörerische Nutzung der Arbeitskraft, der Infrastruktur, des städtischen Raums, der Natur oder der Umwelt durch das Kapital hervor. ${ }^{3}$

Eine ergänzende Lesart des so genannten »zweiten Widerspruchs des Kapitalismus« ist die des Geographen David Harvey (2004), der den von Marx in Das Kapital analysierten Prozess der primitiven Kapitalakkumulation in den Mittelpunkt stellt. Es handelt sich dabei um die Enteignung und Beschlagnahmung des Landes von der Bauernschaft, die dann als Proletarier auf den Arbeitsmarkt geworfen werden. Die Aktualisierung dieser Interpretation, die in der lateinamerikanischen Bibliographie weit verbreitet ist, unterstreicht die Bedeutung der Dynamik der Enteignung im gegenwärtigen Stadium, das sich auf Waren, Personen und Territorien auswirkt. Besagte Interpretation erkennt einen wichtigen Vorläufer im Werk von Rosa Luxemburg, die zu Beginn des 20. Jahrhunderts den kontinuierlichen, nicht ausschließlich mit den Ursprüngen des Kapitalismus verbundenen Charakter der "primitiven sogenannten ursprünglichen Akkumulation« beobachtete.

An zweiter Stelle klärt uns der Neo-Extraktivismus über die Krise des Projektes der Moderne und im Allgemeineren über die aktuelle

3 So haben bereits in den 1970er Jahren marxistische Autoren wie Henri Lefebvre die Notwendigkeit betont, die Lesarten der Dynamik des Kapitals zu erweitern. So appellierte der französische Soziologe angesichts der »verknöcherten Dialektik von Kapital und Arbeit« an eine Dialektik von Kapital, Arbeit und Boden, die nicht nur auf die Naturgewalten, sondern auch auf die damit verbundenen Akteure, einschließlich des Staates, der die Souveränität über ein Staatsgebiet ausübt, verweist. Zitiert in Coronil (2002). 
sozial-ökologische Krise auf. Zweifellos wurde die Bedrohung, die wir durch große anthropogene und soziogene Veränderungen auf planetarer Ebene erleben, die das Leben auf dem Planeten gefährden (Anthropozän), in eine Infragestellung der gegenwärtigen Entwicklungsdynamik übersetzt. Diese ist mit der Kritik der unbeschränkten Erweiterung der Grenze der Kommodifizierung sowie mit der dominanten dualistischen Vision, die der Moderne innewohnt, verknüpft. Folglich ist es möglich, eine Beziehung zwischen dem Neo-Extraktivismus (als dominante Entwicklungsdynamik) und dem Anthropozän (als Kritik an einem bestimmten Modell der Moderne) herzustellen, wenn man seine Konsequenzen auf planetarer Ebene untersucht. Die ökologische Krise erscheint somit untrennbar mit der Krise der Moderne verbunden. In Anlehnung an Arturo Escobar (2005) weist er uns auf die Notwendigkeit hin, an Alternativkonzepte zur Moderne zu denken: Es gilt andere Paradigmen $\mathrm{zu}$ beachten, die die Reproduktion des Lebens in den Mittelpunkt rücken und auf die Wiederherstellung der Verbindung zwischen Menschlichem und Nichtmenschlichem zielen, und zwar aus einer beziehungsorientierten, nicht-dualistischen Perspektive heraus.

Zum Dritten verbindet uns der Neo-Extraktivismus auch mit der Weltwirtschaftskrise, da das gegenwärtige Akkumulationsmodell mit den Reformen des neoliberalen Finanzkapitalismus seit den 1990er Jahren, die sich durch die Finanzkrise von 2008 verschärft haben, verbunden erscheint. Einerseits spielt das Finanzwesen eine grundlegende Rolle bei der Gewinnung von Rohstoffen sowie bei der Organisation der Logistik ihres Umlaufs (Gago und Mezzadra 2015), selbstverständlich auch bei der Preisbestimmung für Rohstoffe an den internationalen Börsen. Andererseits verschärfte die Krise die sozialen Ungleichheiten, die auf einer wirtschaftlichen Strukturanpassungspolitik beruhen, die sich auch auf die Länder der Zentren ausbreitete und die Wirtschaftsmodelle attraktiver machte, die die Natur als Alternative gegen die Rezession stärker kommodifizieren. Auf diese Weise wird von den Ländern der Zentren aus das so genannte integrative Green EconomyModell gefördert, das das Finanzformat des Emissionshandelsmarktes auf andere Naturelemente ausdehnt - wie beispielsweise Luft, Wasser oder Prozesse und Funktionen der Natur (Svampa und Viale 2014). 
Viertens stellt der Neo-Extraktivismus ein privilegiertes Fenster dar, welches uns eine geopolitische Interpretation des relativen $\mathrm{Ab}$ stiegs der Vereinigten Staaten und des Aufstiegs Chinas als globale Macht ermöglicht. Diese Situation eines hegemonialen Übergangs wird als Einstieg in eine Epoche verstanden, die durch konfliktreichen Polyzentrismus und Pluralität in kultureller-zivilisatorischer Hinsicht gekennzeichnet ist und deren Folgen noch nicht genau abzusehen sind. Von den globalisierten Peripherien, sowohl in Lateinamerika und Afrika als auch in einigen Regionen Asiens, korrelierte der Übergang in der geopolitischen Hegemonie mit der Steigerung der Rohstoffexporte, was sich in der Konsolidierung zunehmend ungleicher wirtschaftlicher und sozial-ökologischer Verbindungen, insbesondere mit der Volksrepublik China, zeigt. Mit anderen Worten: Im gegenwärtigen geopolitischen Kontext, der auf das große asiatische Land als neue Weltmacht hindeutet, erlaubt uns der Neo-Extraktivismus, den Prozess der globalen Rekonfiguration zu erfassen. Dieser Prozess wird von den Peripherien aus durch die Erweiterung der Rohstoffgrenzen und einer schwindelerregenden Unterdrückung der produzierenden Wirtschaft umgesetzt.

Last but not least ist der Neo-Extraktivismus also ein privilegiertes Fenster für eine Auslegung im Hinblick auf die Krise der Demokratie, also auf das Verhältnis zwischen politischem Regime, Demokratie und der Einhaltung der Menschenrechte. Sicherlich erscheint der $\mathrm{Zu}$ sammenhang zwischen Neo-Extraktivismus und dem Überschreiten der Demokratiegrenzen als unbestreitbare Tatsache: Ohne soziale Genehmigung, ohne Rücksprache mit der Bevölkerung, ohne Umweltkontrollen und mit geringer Präsenz des Staates - oder sogar mit ihr gibt es eine offenkundige Tendenz der Manipulation der Öffentlichkeit mit dem Ziel, kollektive Entscheidungen zu kontrollieren. Andererseits wirft die Zunahme staatlicher und halbstaatlicher Gewalt die Frage nach den immer spannungsgeladeneren Zusammenhängen zwischen Extraktivismus und Menschenrechten auf. Die perverse Gleichung zwischen »mehr Extraktivismus, weniger Demokratie« (Svampa 2016) zeigt den gefährlichen Übergang zur politischen Stilllegung angesichts der wachsenden Kriminalisierung von sozial-ökologischen Protes- 
ten und den zunehmenden Morden an UmweltaktivistInnen auf der ganzen Welt, insbesondere aber in Lateinamerika.

Zusammenfassend lässt sich sagen, dass der Extraktivismus das lange Gedächtnis des Kontinents und seiner Probleme durchzieht. Er definiert einen Modus der Naturaneignung, ein Muster der kolonialen Akkumulation, das mit der Geburt des modernen Kapitalismus verbunden ist. Seine Aktualisierung im 21. Jahrhundert bringt jedoch neue Dimensionen auf unterschiedlichen Ebenen mit sich: die globale Ebene (hegemonialer Übergang, Erweiterung der Rohstoffgrenzen, Erschöpfung nicht erneuerbarer Naturgüter, sozial-ökologische Krise planetarischen Ausmaßes), die regionale und nationale Ebene (Verhältnis zwischen dem extraktiven Exportmodell, dem Nationalstaat und der Aneignung außerordentlicher Erträge), die territoriale Ebene (stärkere Besetzung des Territoriums, ökoterritoriale Kämpfe mit Beteiligung verschiedener kollektiver Akteure) und schließlich die politische Ebene (Entstehung einer neuen Grammatik des politischen Protests, Zunahme der staatlichen und halbstaatlichen Gewalt).

\section{Neo-Extraktivismus als Entwicklungspfad und sozio- territoriales Modell}

Der zeitgenössische Neo-Extraktivismus kann als Entwicklungsmodell charakterisiert werden, das auf der Überausbeutung immer knapper werdender, größtenteils nicht erneuerbarer Naturgüter sowie auf der Ausweitung der Nutzungsgrenzen auf Gebiete, die zuvor aus kapitalpolitischer Sicht als unproduktiv galten, beruht. Er zeichnet sich durch die Exportorientierung von Rohstoffen in großem Maßstab aus, darunter Kohlenwasserstoffe (Gas und Öl), Metalle und Mineralien (u.a. Kupfer, Gold, Silber, Zinn, Bauxit, Zink) sowie Produkte, die mit dem neuen landwirtschaftlichen Paradigma verbunden sind (Sojabohnen, Ölpalmen, Zuckerrohr). Der so definierte Neo-Extraktivismus bezeichnet also mehr als nur die traditionell als extraktiv betrachteten Aktivitäten. Dazu gehören der Tagebau, die Erweiterung der Öl- und Energiegrenze, der Bau großer Staudämme und anderer Infrastrukturprojek- 
te - unter anderem Autobahnen, Häfen, bi-ozeanische Korridore - bis hin zur Expansion verschiedener Formen von Monokulturen oder Monoproduktion durch die Generalisierung des Agrar-Geschäftsmodells, Überfischung oder Forstmonokulturen.

In diesem Sinne ist der Neo-Extraktivismus auch ein gesellschaftspolitisches und territoriales Modell, das auf nationaler, regionaler oder lokaler Ebene plausibel analysiert werden kann. So führte beispielsweise die Expansion der Sojagrenze in mehreren südamerikanischen Ländern zu einer Rekonfiguration der ländlichen Gebiete:

»Allein zwischen 2000 und 2014 vergrößerten sich die Sojaplantagen in Südamerika um 29 Millionen Hektar, was in etwa der Fläche Ecuadors entspricht. Brasilien und Argentinien machen etwa 90\% der regionalen Produktion aus, obwohl die schnellste Expansion in Uruguay stattgefunden hat, und Paraguay ist das Land, in dem Soja im Vergleich zu anderen Anbaukulturen die größte Fläche einnimmt, nämlich $67 \%$ der gesamten landwirtschaftlichen Fläche« (Oxfam 2016, 30).

Ein weiteres wichtiges Merkmal des Neo-Extraktivismus ist der Gigantismus oder das große unternehmerische Ausmaß, das uns auch vor dem Umfang der Investitionen warnt, da es sich nicht um arbeitsintensive, sondern um kapitalintensive Megaprojekte handelt. Dies bezieht sich auf den Charakter der beteiligten Akteure - im Allgemeinen große transnationale Konzerne -, auch wenn natürlich die so genannten »Translatinas « nicht ausgeschlossen sind, sprich nationale MegaUnternehmen wie beispielsweise Petróleos de Venezuela (PDVSA) und auch Argentiniens Yacimientos Petrolíferos Fiscales (YPF). Gleichzeitig warnt uns dies vor einer wichtigen Variable in Bezug auf die Großprojekte: der geringen Generierung von direkten Arbeitsplätzen (deren Maximum in der Bauphase des Projekts erreicht wird). So werden beispielsweise beim groß angelegten Bergbau pro Million investierter Dollar nur 0,5 bis 2 direkte Arbeitsplätze geschaffen (Colectivo Voces de Alerta, 2011). In Peru, dem Land schlechthin im transnationalen MegaMining, sind nur $2 \%$ der erwerbstätigen Bevölkerung im Bergbau beschäftigt. Demgegenüber stehen $23 \%$ in der Landwirtschaft, $16 \% \mathrm{im}$ 
Handel und fast $10 \%$ in der Fertigungsindustrie (Lang und Mokrani 2012).

Ebenso stellt der Neo-Extraktivismus eine gewisse territoriale Dynamik dar, deren Tendenz die massive Besetzung von Territorium und Land durch mit Monokultur oder Monoproduktion verbundene Wirtschaftsformen ist. Eine ihrer Folgen ist die Verdrängung anderer Produktionsformen, besonders von lokalen und regionalen Ökonomien, sowie der lokalen Bevölkerungen. In dieser Hinsicht hat der Neo-Extraktivismus zu Beginn des 21. Jahrhunderts den Landkonflikt neu definiert. Er konfrontiert asymmetrisch arme und gefährdete Bevölkerungsgruppen mit großen Wirtschaftsakteuren, die an der Implementierung gentechnisch veränderter Kulturen in Verbindung mit Sojabohnen, Palmöl, Zuckerrohr und anderem interessiert sind. Laut dem Oxfam-Bericht (mit Daten aus landwirtschaftlichen Erhebungen in 15 Ländern) »konzentrieren sich in der gesamten Region $1 \%$ der größten Betriebe auf mehr als die Hälfte der landwirtschaftlichen Fläche. Mit anderen Worten: 1\% der Betriebe haben mehr Land als die restlichen $99 \%$. Kolumbien ist das Land mit der ungleichsten Landverteilung, wo $0,4 \%$ der Landwirtschaftsbetriebe $68 \%$ der landwirtschaftlichen Nutzfläche des Landes dominieren, gefolgt von Peru, wo $77 \%$ der landwirtschaftlichen Betriebe in den Händen von $1 \%$ sind, dann Chile (74\%), Paraguay (71\%), Bolivien, wo $1 \% 66 \% \mathrm{der}$ landwirtschaftlichen Betriebe verwaltet, Mexiko mit $56 \%$, Brasilien mit $44 \%$ der landwirtschaftlichen Nutzfläche für 1\% der Betriebe. In Argentinien befinden sich $36 \%$ der Ackerfläche in den Händen von Großgrundbesitzern und Saatgut-Pools. ${ }^{4}$ Für Gian Carlo Delgado gilt:

»Die Landenteignung sollte als die Aneignung von Land betrachtet werden, das für 1) Monokulturen bestimmt ist, einschließlich der

4 Die Daten von Oxfam (2016) wurden im November 2016 veröffentlicht. Es ist klarzustellen, dass sie sich auf die Bewirtschaftung und nicht auf die Menschen beziehen; daher berücksichtigen sie keine landbesitzlosen Bauern und liefern nur sehr wenige Informationen über das kollektive Eigentum (für die Fälle Bolivien, Kolumbien und Peru). Siehe auch Aranda (2017). 
so genannten >Wild Card oder flex crops (Nahrungsmittel/Bioenergie/Produktionsmittel; z.B. Mais, Zuckerrohr, Ölpalmen) und die Produktion von Non-Food-Inhalten wie Zellulose; 2) den Zugang, die Bewirtschaftung und die Nutzung von Ressourcen wie Energie- und Nichtenergie-Mineralien sowie 3) Trinkwasser (oder Blue-Grabbing) und für 4) die Erhaltung oder so genannte grüne Flächennutzung (Green Grabbing), die von der Schaffung privater Schutzgebiete bis zur Umsetzung von Klimaschutzprojekten wie dem so genannten REDD+ (Projekte zur Verringerung der Emissionen aus der Abholzung und des Abbaus und für die Erhaltung) reicht.« (Delgado 2016, 54).

\section{"Rohstoff-Konsens" und Entwicklungsillusionen}

In Lateinamerika expandierte der Neo-Extraktivismus in einem Kontext des epochalen Wandels vom neoliberalen Washington-Konsens, der mit Geldwertstabilität und Strukturanpassung verbunden ist, zum Rohstoff-Konsens. Letzterer ist mit dem großangelegten Export von Primärgütern, dem Wirtschaftswachstum und der Ausweitung des Konsums verbunden (Svampa, 2013). Im Gegensatz zu den 1990er Jahren wurden die lateinamerikanischen Ökonomien im Zeitraum von 2000-2003 durch hohe internationale Rohstoffpreise begünstigt, die sich durchweg in positiven Handelsbilanzen und Haushaltsüberschüssen niederschlugen. Diese Tatsache darf nach der langen wirtschaftlichen Stagnation und angesichts des Rückschritts der vergangenen Jahrzehnte, insbesondere der offen neoliberalen Periode in den 1990er Jahren, nicht ignoriert werden. In dieser günstigen Wirtschaftslage zumindest bis 2013 - tendierten lateinamerikanische Regierungen da$\mathrm{zu}$, die komparativen Vorteile des Rohstoffbooms hervorzuheben und die neuen sozio-ökologischen Ungleichgewichte und Asymmetrien zu leugnen oder zu beschönigen. Diese Ungleichgewichte gingen mit der Vertiefung eines Entwicklungsmodells einher, das auf dem Rohstoffexport im großen Stil basiert. In diesem Sinne ermöglichten alle lateinamerikanischen Regierungen - unabhängig von ihrer eigenen linken Orientierung - die Rückkehr eines produktivistischen Verständnisses 
von Entwicklung, und die Wiederbelebung der Entwicklungsillusion. Dies ging einher mit der Leugnung und Verschleierung grundlegender Diskussionen über die sozialen, ökologischen, territorialen, politischen und neo-extraktivistischen Auswirkungen sowie der Diffamierung von sozial-ökologischen Mobilisierungen und Protesten.

In Bezug auf die Konsequenzen war der Rohstoffkonsens (consenso de los commodities) durch eine komplexe, rasante und rückläufige Dynamik gekennzeichnet, die aus einer Vielzahl von Perspektiven betrachtet werden muss. Aus wirtschaftlicher Sicht trat er etwa durch eine neue Tendenz zur Reprimarisierung der Ökonomie in Erscheinung, was sich in der Neuausrichtung auf den primärwirtschaftlichen Bergbau mit geringer Wertschöpfung zeigt. Dieser »Reprimarisierungseffekt« wurde durch den wirtschaftlichen Auftritt Chinas verstärkt, einer Macht, die sich schnell als ungleicher Partner in der gesamten lateinamerikanischen Region durchgesetzt hat. 2014 reichten die Exporte von Primärgütern in den Mercosur-Ländern von 65 \% (Brasilien) bis 90 \% (Paraguay) (Cepal 2015). ${ }^{5}$ Selbst ein Land wie Brasilien, das über eine diversifizierte Volkswirtschaft verfügt, litt aus diesem Grund unter dem, was der französische Ökonom Pierre Salama (2011) als Phänomen der »frühen Deindustrialisierung« bezeichnete.

Beim genannten Rohstoff-Konsens können sowohl Brüche als auch Kontinuitäten im Vergleich zur Vorperiode des Konsenses von Washington beobachtet werden. Die Politik des Washington-Konsenses

5 Laut H.-J. Burchardt sind drei regionale Dynamiken im Zusammenhang der Expansion der extraktivistischen Ökonomien in Lateinamerika zu unterscheiden. Auf der einen Seite stehen die Länder, die sich durch ihre Tendenz zur Monoproduktion, also durch den Export hauptsächlich eines Rohstoffs auszeichnen, wie Ecuador und Venezuela (Öl), Peru und Chile (Bergbau) und Bolivien (Gas). Auf der anderen Seite gibt es Länder, die über eine diversifizierte Wirtschaft verfügen, aber effektiv die mineralgewinnenden Sektoren ausgebaut haben, etwa Brasilien mit Bergbau, Soja und nun auch Öl durch die so genannte PreSalt-Cewinnung. Schließlich gibt es die Länder Mittelamerikas sowie Mexiko, die in der ersten Phase des Rohstoffkonsenses nicht komplett auf Extraktivismus gesetzt hatten, sich aber eindeutig in diese Richtung bewegen (Burchardt 2016, 63). 
führte dazu, dass der Staat als meta-regulatorischer Akteur neu definiert wurde. Ebenso erfolgte mit dem Neoliberalismus eine Art politische Homogenisierung in der Region, die durch eine starke Nähe zu den Strategien der Weltbank gekennzeichnet war. Im Gegensatz dazu stellte der Rohstoffkonsens die massive Umsetzung exportorientierter Rohstoffprojekte in den Mittelpunkt und schuf einen flexibleren Raum für die Rolle des Staates. So waren Entfaltung und Koexistenz zwischen progressiven Regierungen, die den neoliberalen Ansatz in Frage stellten, und jenen anderen Regierungen, die eine konservative politische Matrix im Rahmen des Neoliberalismus vertieften, möglich. Zweifellos ist der Rohstoffkonsens in der progressiven Anschauung mit staatlichem Handeln wie einer Reihe von Wirtschafts- und Sozialpolitiken für die am meisten benachteiligten Sektoren der Gesellschaft verbunden. Die materielle Grundlage für diese Maßnahmen beruht auf dem außerordentlichen Einkommen, das durch den extraktivistischen und exportbasierten Ansatz generiert wurde. In diesem Kontext konnten einige institutionelle Instrumente und staatliche $\mathrm{Ka}$ pazitäten zurückgewonnen werden; wobei der Staat wieder zu einem relevanten Akteur für sozio-politische Regulierungen bis hin zu sozioökonomischen Umverteilungspolitiken werden konnte. Jedoch sehen Governance-Theorien, die auf supranationale Rahmenbedingungen des Institutionenaufbaus hinweisen, nicht gerade eine Tendenz hin zum Nationalstaat als Megainstanz oder zur Garantie grundlegender Veränderung. Im Gegenteil deutet die maximale Hypothese eher auf die Rückkehr eines in Maßen regulierenden Staates hin, der in der Lage ist, sich in einen Raum variabler Strukturen und Ebenen einzufügen, in denen auch verschiedene zivilgesellschaftliche Akteure intervenieren. Dazu gehören soziale Bewegungen und Nichtregierungsorganisationen (NGOs), aber auch andere Akteure, die ihrerseits jedoch oft in enger Verbindung mit multinationalen Metropolen stehen, deren Einfluss in den lateinamerikanischen Volkswirtschaften keineswegs zurückgegangen ist, sondern deutlich zugenommen hat. Obwohl der progressive Ansatz anderer Meinung war und in Hinblick auf die richtungsweisende Rolle des Staates vom Neoliberalismus abweicht, war der Staat, wie der argentinische Ökonom Mariano Feliz betont, 
weit davon entfernt, die Hegemonie des transnationalen Kapitals in der peripheren Wirtschaft in Frage zu stellen (Feliz 2012, 24-27). Diese Tatsache setzte dem Handeln des Nationalstaates klare Grenzen und stellte eine unüberwindbare Schwelle für die Forderung nach Demokratisierung kollektiver Entscheidungen dar, welche gerade von den durch die großen Rohstoffprojekte betroffenen comunidades und Bevölkerungsgruppen erhoben wurde.

Auf der anderen Seite ist anzumerken, dass in Lateinamerika weiterhin ein großer Teil des linken politischen Spektrums und der populistischen Progressisten eine produktivistische Vision von Entwicklung unterstützt ${ }^{6}$, die den sozialen Konflikt im Sinne des Gegensatzes von Kapital und Arbeit interpretiert und die Beziehungen Kapital-Natur sowie die sozialen Kämpfe, die sich auf die Verteidigung von Territorien und Gemeinschaftsgütern konzentrieren, banalisiert oder ignoriert. In diesem Zusammenhang, besonders zu Beginn des progressiven Zyklus, wurde die Dynamik der sozialen Enteignung tendenziell zu einem blinden Fleck, der nicht mehr denkbar war. Davon ausgehend schienen sozio-ökologische Problematiken gegenüber den strukturellen Problemen von Armut und sozialer Ausgrenzung in den lateinamerikanischen Gesellschaften als nachrangig, ja vernachlässigbar. So hing der überwiegende Teil der lateinamerikanischen Linken - trotz der durch sie vorangetriebenen Wiederbelebung indigen-kommunitärer Modelle - nach wie vor einer auf Produktion und Effizienz fokussierenden Idee von Entwicklung an, die der hegemonialen Fortschrittsideologie eng verbunden blieb und auf die Ausweitung der Produktivkräfte setzte.

In diesem Sinne rechtfertigten die progressiven Regierungen den Neo-Extraktivismus als Königsweg für die Generierung von Devisen

6 Der Produktivismus gründet auf der Idee des unbegrenzten Wachstums und impliziert eine Nichtanerkennung der Crenzen der Nachhaltigkeit auf der Erde. Eine ausgezeichnete Definition liefert Joaquín Sempere, indem er erklärt, dass »der Begriff >Produktivismus jeden sozialen Stoffwechsel bezeichnet, der die Grenzen der ökologischen Nachhaltigkeit nicht einhält, weil er der Ansicht ist, dass sich die Menschheit erlauben kann, natürliche Ressourcen nach Belieben und ohne Grenzen zu nutzen« (Sempere 2015). 
für den Staatshaushalt, die Umverteilung von Einkommen und Ankurbelung des Binnenkonsums oder die Diversifizierung der Ökonomien. Dieser Ansatz, der sozialen Frage (d.h. Umverteilung, Sozialpolitik) das Umweltproblem (d.h. Erhaltung von Gemeingütern, Pflege von Gebieten) entgegenzusetzen und dabei komplexe und grundlegende Diskussionen über Entwicklung, ökologische Nachhaltigkeit und Demokratie auszulassen, muss in seinen jeweils verschiedenen Ausformungen untersucht werden. Generell aber kann gesagt werden, dass die lateinamerikanischen Regierungen unter dem Deckmantel der »komparativen Vorteile« versuchten, ein konsumorientiertes Inklusionsmodell voranzubringen. Diese Kopplung zwischen dem Vordringen des Staates und dem Modell des Bürgers als Konsument war die Voraussetzung für einen möglichen Wahlerfolg und für die Aufrechterhaltung der politischen Macht der verschiedenen Regierungen.

Der Fakt, dass Lateinamerikas Wirtschaft sich als anpassungsfähig gegenüber den unterschiedlichen Akkumulationszyklen gezeigt und die Region somit ihre Position in der globalen Arbeitsteilung letztendlich akzeptiert hat, stellt eines der ungelösten Kernelemente sowohl des Washingtoner- als auch des Rohstoff-Konsens dar. Dies gilt ganz ungeachtet der politischen Rhetorik der progressiven Regierungen, die wirtschaftliche Autonomie und nationale Souveränität für sich beanspruchen und einen politischen Integrationsprozess in Lateinamerika reklamieren. Letztlich wurde das dominante Entwicklungsmodell nicht nur durch eine instrumentale und produktivistische Vision getragen, sondern auch durch die Neuauflage sozialer Imaginarien geprägt, die den (historischen) Überfluss an natürlichen Ressourcen in der Region predigen (eine Vorstellung von Lateinamerika als Eldorado). In manchen Ländern stand diese Vorstellung im Zusammenhang mit der Krisenerfahrung, das heißt mit dem ausgrenzenden Erbe der 1990er Jahre, das zu immer größeren Ungleichheiten und Armut führte. So sprach etwa der ehemalige ecuadorianische Präsidenten Rafael Correa vom Ende der »langen neoliberalen Nacht«, und bezog sich damit auf die tiefe Krise der ersten Jahre des 21. Jahrhunderts (Arbeitslosigkeit, Chancenminderung, Migration, politische Instabilität). Dieses Bild erschien auch in Argentinien immer wieder im politischen Diskurs von Néstor und 
Christina Fernández de Kirchner über »el país normal«. Hierbei ging es darum, die wirtschaftlichen und sozialen Indikatoren ihrer jeweiligen Regierungen mit den neoliberalen Jahren (1990er Jahre, unter dem neoliberalen Kurs von Carlos Menem) und vor allem mit denen der großen Krise $\mathrm{zu}$ vergleichen, die das Land zwischen 2001 und 2002 erschütterte und in einem Aussetzen der Konvertibilität zwischen Peso und US-Dollar mündete.

So nahm Lateinamerika in einer neuen Phase der Expansion der Kapitalgrenzen den ursprünglichen Mythos des unerschöpflichen Ressourcenreichtums wieder auf und stützte sich dabei auf ein Wunschdenken (Zavaletta 2009). Diese Entwicklungsillusion war getragen von der Idee, im Zuge der guten wirtschaftlichen Aussichten (d.h. der Erhöhung der Rohstoffpreise und der wachsenden Nachfrage, vor allem aus China) die Distanz zu den Industrieländern schnellstmöglich überwinden zu können, und so die stets versprochene und nie realisierte Entwicklung unserer Gesellschaften zu erreichen. Ob in der Form einer groben Rhetorik der kapitalistischen Landnahme (liberale Perspektive) oder einer die staatliche Kontrolle des Überschusses betonenden Rhetorik (progressive Perspektive): die aktuellen, auf dem extraktivistischen Paradigma basierenden Entwicklungsmodelle haben die Vorstellung von Lateinamerika als Eldorado, das die Geschichte des Kontinents prägt, vergegenwärtigt.

Folglich zeigte sich in dem lateinamerikanischen Szenario nicht nur eine Kopplung zwischen Neo-Extraktivismus, Entwicklungsillusion und Neoliberalismus, die in paradigmatischer Weise in den Fällen von Peru, Kolumbien oder Mexiko zum Ausdruck kam, sondern auch zwischen Neo-Extraktivismus, Entwicklungsillusion und progressiven Regierungen. Dies hat die Beziehungen zwischen Linksregierungen und den indigenen sowie sozio-ökologischen Bewegungen kompliziert und problematisch gemacht. Die paradoxesten lateinamerikanischen Beispiele des Rohstoffkonsenses während der Hochphase des progressiven Kurses waren Bolivien und Ecuador. Das Thema ist nicht unbedeutend, denn in diesen Ländern wurden im Rahmen intensiver Beteiligungsprozesse neue Konzepte wie der plurinationale Staat, Autonomien, buen vivir und Naturrechte ins Leben gerufen, die sich auch 
in den neuen verabschiedeten Verfassungen wiederfinden. Mit der Konsolidierung dieser Regime gewannen allerdings auch andere Fragen an Bedeutung, die mit dem Rohstoffexport und dessen Bedeutung für das Wirtschaftswachstum zusammenhängen.

Nach meinem Verständnis hat der Rohstoffkonsens auch eine politisch-ideologische Bedeutung, da er auf die Idee anspielt, dass es eine implizite oder explizite Übereinkunft über die Alternativlosigkeit der gegenwärtigen extraktivistischen Dynamik gäbe, die aus der wachsenden globalen Nachfrage nach Primärgütern resultiere. Wie schon in den goldenen Jahren des Neoliberalismus, also den 1980er und 1990er Jahren, als der O-Ton lautete, dass es keine Alternative zum Neoliberalismus gebe, behaupteten die politischen Eliten der Region (Progressive und Konservative) ab der Jahrtausendwende, dass es keine Alternative zum Extraktivismus gebe. Es entstand ein Grundsatz der »Vernunft und Angemessenheit « (sensatez y razonabilidad), der den verschiedenen Versionen des progressiven Kapitalismus eigen wurde. So wurden kollektive Widerstände in die Schranken gewiesen und gleichzeitig ein neuer, historisch begründeter Schutzschirm gegen die Herausbildung grundlegender Alternativen aufgebaut. Wie Mirta Antonelli festhält, zielt das "Durchsetzen einer exklusiven Erzählung der möglichen Welt « darauf, andersartige Logiken zu kontrollieren und zu neutralisieren, und somit »andere Argumente, andere Beweggründe, andere Erinnerungen und Gefühle, andere gesellschaftliche Projekte« zu entkräften (Antonelli 2011, 11). In der Folge wird jeder kritische Diskurs oder jede radikale Opposition in den Bereich der Irrationalität, Antimodernität, der Verweigerung des Fortschritts, des pachamamismo, oder des infantilen Ökologismus abgedrängt oder gar als kolonialer Umweltschutz, der angeblich immer von NGOs oder ausländischen Akteuren gefördert wird, diffamiert. So war das neue Jahrhundert im Gegensatz zu den 1990er Jahren, als sich der Kontinent durch das neoliberale Modell in eine einzige Richtung zu bewegen schien, von einer Reihe von Spannungen und Widersprüchen geprägt, die schwer zu durchbrechen waren. Der Übergang vom Washingtoner Konsens zum Rohstoffkonsens brachte neue Probleme und Paradoxien mit sich, die sogar den Hori- 
zont des lateinamerikanischen kritischen Denkens und der gesamten Linken neugestalteten.

Kurz gesagt, zeigt das lateinamerikanische Szenario über die festgestellten politisch-ideologischen Unterschiede und Nuancen hinaus die Vertiefung eines Modells der Aneignung und der Ausbeutung von Gemeingütern, das von einer vertikalen Logik (von oben nach unten) über die Bevölkerung hinweg ausgeht, die Fortschritte im Bereich der partizipativen Demokratie stark ins Wanken bringt und einen neuen Teufelskreis der Kriminalisierung und Verletzung der Menschenrechte angekurbelt hat. 



\section{Kapitel 2: Sozio-ökologische Konflikte und Sprachen der Inwertsetzung}

In diesem Kapitel wird der sozio-ökologische Konflikt auf seinen verschiedenen Ebenen behandelt. Zunächst wird die rekursive Dynamik der Konflikte historisiert, indem ich verschiedene Phasen des NeoExtraktivismus analysiere. Gleichzeitig beschäftige ich mich mit den Merkmalen der neuen Sprache der Bewertung von Territorium, die im Zuge dieser Konflikte entstanden ist und die ich als ökoterritoriale Wende bezeichne.

\section{Phasen des Neo-Extraktivismus}

Eine der Folgen der gegenwärtigen extraktivistischen Flexion ist die explosionsartige Zunahme sozio-ökologischer Konflikte, was in der Verschärfung uralter Landkonflikte - mit indigenen und bäuerlichen Bewegungen in der Hauptrolle - sowie in der Entstehung neuer Formen der Mobilisierung und Partizipation der Bevölkerung zum Ausdruck kommt. Letztere konzentrieren sich auf die Bewahrung des Gemeinwesens, der Biodiversität und der Umwelt. Die Konfliktträchtigkeit des Neo-Extraktivismus ist dabei nicht als ein mögliches Ergebnis, sondern als inhärenter Bestandteil desselben zu verstehen, da er sich ja durch soziale Fragmentierung, Verdrängung anderer Wirtschaftsformen, vertikale Entscheidungsstrukturen und starke Auswirkungen auf die Ökosysteme auszeichnet (auch wenn neo-extraktivistische Politik nicht überall zwangsläufig zu sozialem Widerstand führt). 
Ich verstehe unter sozio-ökologischen Konflikten diejenigen, die mit dem Zugang zu und der Kontrolle von Naturgütern und Territorien verbunden sind. Diese Konflikte implizieren also je unterschiedliche Interessen und Werte seitens der Konfliktparteien in einem Kontext großer Machtasymmetrie. ${ }^{1}$ Diese Konflikte drücken unterschiedliche Auffassungen von Territorium, Natur und Umwelt aus. In einigen Fällen, in dem mehrere Megaprojekte dazu neigen, das Territorium als Ganzes neu zu gestalten, führen Konflikte letztendlich zu einem Streit darüber, was unter Entwicklung und Demokratie zu verstehen ist.

Im Laufe der Jahre und im Zuge der Ausdehnung der Kapitalgrenze vermehrten sich auch die Konflikte, während die sozialen Widerstände aktiver und organisierter wurden. Auf dieser Grundlage schlage ich vor, zwischen drei Phasen des Neo-Extraktivismus zu unterscheiden.

Die erste ist die Positive Phase, die sich zwischen 2003 und 20082010 entwickelt hat. Zu Beginn des Epochenwechsels und in Anbetracht des Rohstoffpreisbooms wurde die extraktivistische Wende sicherlich in Bezug auf die komparativen Vorteile und über die Unterschiede der progressiven und konservativen Regierungen hinaus als »New Developmentalism« betrachtet. Ich betone, dass es sich um eine Phase der Positivität handelt, denn die Erhöhung der Sozialausgaben und ihre Auswirkungen auf die Armutsbekämpfung, die wachsende Rolle des Staates und die zunehmende Partizipation der Bevölkerung haben große politische Erwartungen in der Gesellschaft geweckt, insbesondere nach den vorigen aufeinanderfolgenden Krisen und Jahrzehnten wirtschaftlicher Stagnation und neoliberaler Anpassung. Nicht zu vergessen ist, dass die Armut in der Region zwischen 2002 und 2011 von $44 \%$ auf $31,4 \%$ gesunken ist, während die extreme Armut von 19,4 \% auf 12,3\% verringert werden konnte (Cepal 2012). Die meisten Länder haben die Anzahl der Sozialpläne erweitert, die $19 \%$ der Bevölkerung erreichen sollen (Cepal 2013); das sind etwa 120 Millionen Menschen die von Sozialprogrammen profitieren.

$1 \quad$ Ich berufe mich auf die Definition von Fontaine (2003), der ich den Hinweis auf den asymmetrischen Charakter der Konflikte hinzufüge. 
Andererseits war diese erste Phase des Neo-Extraktivismus durch eine Art Erweiterung der juristischen Grenzen gekennzeichnet, die sich in der Schaffung neuer Rechte (sowohl individuell als auch kollektiv) zeigt. Diese Staatsnarrative koexistierten mit ihren Artikulationen und Spannungen mit indigenistischen und ökologischen Narrativen, die sich etwa in Bolivien und Ecuador neben der neuen Hegemonie der staatspopulistischen Matrix etablierten. Im Laufe des Jahrzehnts und im Zuge verschiedener territorialer und sozio-ökologischer Konflikte und ihrer rekursiven Dynamik nahmen jedoch auch progressive Regierungen zunehmend einen aggressiven entwicklungspolitischen Diskurs zur Verteidigung des Extraktivismus an. Dieser wurde Praxis der Kriminalisierung und Repression sozial-ökologischer Konflikte sowie von einem ausdrücklichen Willen zur Kontrolle der Partizipationsformen der Bevölkerung begleitet.

Diese Phase des Wirtschaftsbooms und der Neuformulierung der Rolle des Staates war auch eine Periode mangelnder Sichtbarkeit, wenn nicht gar der Nichtanerkennung der Konflikte im Zusammenhang mit der extraktiven Dynamik, die sich etwa bis 2008-2010 erstreckte. $\mathrm{Zu}$ dieser Zeit bekräftigten die progressiven Regierungen, die durch Wiederwahl in ihren Mandaten bestätigt worden waren (viele von ihnen bei Präsidentschaftswahlen), eine explizit extraktivistische Grundposition, welche der Ausgangspunkt für eine Vielzahl virulenter territorialer und sozial-ökologischer Konflikte wurde. Darüber hinaus legte der Ausbruch von Konflikten im Zusammenhang mit extraktiven Aktivitäten (Mega-Bergwerke, Mega-Staudämme, Ölförderung, Erweiterung der Agrargrenze) sowohl die dem hegemonialen Entwicklungsmodell innewohnenden Formationen und Allianzen offen als auch die Einschränkung von partizipativen Prozessen sowie Tendenzen zur verstärkten Kriminalisierung der Proteste.

Die zweite Phase ist gekennzeichnet durch die Multiplikation der Megaprojekte sowie die Vervielfachung der sozialen Widerstände. In Bezug auf erstere spiegelt sich dies in den von den verschiedenen Regierungen vorgelegten Nationalen Entwicklungsplänen wider, deren Schwerpunkt in allen Fällen auf der Zunahme der verschiedenen Extraktionsaktivitäten je nach Ausrichtung des Landes lag: Abbau von Mineralien, 
Öl, Wasserkraftwerke oder der Ausbau von gentechnisch veränderten landwirtschaftlichen Produkten. Im Falle Brasiliens war dies der 2007 gestartete Plan zur Beschleunigung des Wachstums (Programa de Aceleração do Crescimento), der den Bau einer großen Anzahl von Dämmen im Amazonasgebiet sowie die Durchführung von Mega-Energieprojekten im Zusammenhang mit der Gewinnung von Öl und Gas vorsah. Für Bolivien war es das Versprechen eines großen industriellen Sprungs, der unter anderem auf der Vervielfachung von Projekten zur Gewinnung von Gas, Lithium und Eisen sowie dem Ausbau des Agrarsektors beruhte; für Ecuador die Öffnung für den Tagebau sowie die Ausdehnung der Ölgrenze; für Venezuela der strategische Ölförderplan, der einen Vorstoß an der Abbaugrenze im Orinoco-Gürtel bedeutete; für Argentinien der Strategische Agrarnahrungsmittelplan (Plan Estratégico Agroalimentario 2010-2020), der eine Steigerung der Getreideproduktion um $60 \%$ vorsah, sowie ab 2012 der Plan zur Ausweitung der Öl- und Gasförderung durch Fracking. So war die Staatspolitik der verschiedenen Regierungen auch bei vorgeblich industrieller Rhetorik darauf ausgerichtet, das neo-extraktivistische Modell - dieses galt damals noch als äußerst rentabel - zu vertiefen.

Diese Zunahme von Megaprojekten wurde auch durch die Initiative zur Integration der südamerikanischen regionalen Infrastruktur (Iniciativa para la Integración de la Infraestructura Regional Suramericana, IIRSA), später Consejo Suramericano de Infraestructura y Planeamiento (Cosiplan) genannt, zum Ausdruck gebracht. Diese umfasst Projekte in den Bereichen Verkehr (u.a. Wasserstraßen, Häfen, bi-ozeanische Korridore), Energie (große Wasserkraftwerke) und Kommunikationsinfrastruktur. Es handelt sich um ein Programm, auf das sich mehrere lateinamerikanische Regierungen um das Jahr 2000 geeinigt haben, dessen Hauptziel es ist, den Abbau von Rohstoffen und den Export zu den Zielhäfen zu erleichtern. Seit 2007 gehört die IIRSA zur Union der Südamerikanischen Nationen (UNASUR). Wie Silvia Carpio vom Centro de Estudios y Documentación Latinoamericanos (2017) bestätigt, war der brasilianische Präsident Lula da Silva die treibende Kraft hinter UNASUR und Cosiplan. Er versuchte, die Beziehungen zu anderen südamerikanischen Ländern durch die Intensivierung des regionalen Handels und 
Investitionen der Brasilianischen Entwicklungsbank (BNDS) in Infrastrukturprojekte zu stärken. In verschiedenen Regionen wurden jedoch Projekte des IIRSA-Cosiplan in Frage gestellt oder abgelehnt, da trotz des lateinamerikanistischen Diskurses über die Notwendigkeit, »neue Beziehungen zwischen Völkern und Gemeinschaften aufzubauen«, die so genannte Infrastrukturintegration der IIRSA vor allem kommerziellen Interessen folgte. Dabei handelt es sich um 544 Projekte mit einer geschätzten Investitionssumme von 130 Milliarden U.S.-Dollar. Bis 2014 waren 32,3\% der Investitionen innerhalb von IIRSA für den Energiebereich reserviert, der sich hauptsächlich auf Wasserkraftwerke konzentrierte, die wegen ihrer sozialen und ökologischen Auswirkungen stark in Frage gestellt wurden, insbesondere in der ohnehin schon fragilen Region des brasilianischen und bolivianischen Amazonasgebiets (Carpio 2017). Von den 31 vorrangigen Cosiplan-Projekten betreffen 14 das Amazonasgebiet (Porto 2017, 158).

Diese zweite Phase bringt uns in eine Zeit, in der der Rohstoffkonsens die immer offeneren Konflikte in den Fördergebieten $\mathrm{zu}$ übertünchen suchte. In der Realität aber traten nun zahlreiche sozioökologische und territoriale Konflikte aus dem jeweiligen lokalen Kontext heraus und erlangten eine nationale Sichtbarkeit: Angefangen beim Bau einer Verbindungsstraße in Bolivien, die das indigene Territorium und den Isiboro-Sécure-Nationalpark (Tipnis) durchqueren sollte, über den Bau des Belo-Monte-Mega-Damms (Brasilien), den Aufstand in der Sierra de Famatina gegen den Mega-Bergbau (Argentinien, 2012) bis zur endgültigen Suspendierung des Yasuní-Vorschlags (Ecuador, 2013). Hier wird deutlich, dass die Ausdehnung der juristischen Grenze und die Gewährung neuer kollektiver, territorialer und ökologischer Rechte ihren Endpunkt in der wachsenden Expansion der Grenzen der Kapitalausbeutung auf immer weitere Güter, Landflächen und Territorien gefunden hat. Die hohen Erwartungen, die die emanzipatorischen Narrative gerade in Bolivien und Ecuador geweckt hatten, wurden dadurch bitter enttäuscht.

Neben diesen symbolischen Konflikten in Ländern mit progressiven Regierungen müssen wir auch diejenigen in den Blick nehmen, die gleichzeitig unter neoliberalen oder konservativen Regierungen 
stattgefunden haben, etwa das jetzt eingestellte Bergbauprojekt Conga in Peru, den Widerstand gegen das Minen-Megaprojekt La Colosa im kolumbianischen departamento Tolima, das 2017 endgültig suspendiert wurde, oder den Bau des Staudamms Agua Zarca am Río Gualcarque in Honduras, der letztlich dank der Aktionen des von der 2016 ermordeten Berta Cáceres gegründeten Consejo Cívico de Organizaciones Populares e Indigenas de Honduras (COPINH) ausgesetzt wurde.

Kurz gesagt, in der Hitze der verschiedenen Territorial- und Umweltkonflikte und ihrer rekursiven Dynamik nahmen lateinamerikanische Regierungen einen aggressiven entwicklungspolitischen Diskurs zur Verteidigung des Neo-Extraktivismus an, der die produktivistischen und eldoradistischen Narrative mit einer offenen Praxis der Kriminalisierung von Widerständen begleitete. Diese Angleichung von Diskurs und Praxis, die auch in den Ländern auftrat, die die größten Erwartungen an den politischen Wandel geweckt hatten, insbesondere in Bezug auf die Versprechen des buen vivir im Zusammenhang mit der Naturbewahrung (wie Ecuador und Bolivien), veranschaulichte die Entwicklung progressiver Regierungen hin $\mathrm{zu}$ traditionelleren Herrschaftsmodellen (im Sinne des klassisch-populistischen oder nationalstaatlichen Modells). Ebenso ging diese politische Transformation mit dem Beginn einer neuen Phase der Einschränkung der Grenzen der Demokratie einher, was sich in der wachsenden Intoleranz gegenüber dissidenten Positionen zeigt.

Die Stigmatisierung der Umweltproteste war sicherlich in den verschiedenen progressiven Regierungen ein verbindendes Element; in einigen Fällen wurden die Proteste gar als Verschwörungen deklariert. In Wirklichkeit stellten sich die Behörden einhellig gegen jede Form von ökologischem und territorialem Konflikt, sobald dieser medialisiert und politisiert wurde und die blinden Flecken progressiver Regierungen in Bezug auf die Dynamiken kapitalistischer Landnahme und der Akkumulation durch Enteignung offenlegte. Dies war der Fall in Ecuador ab 2009, insbesondere in Bezug auf das Mega-Mining, in Brasilien nach dem Konflikt um den Bau des Staudamms am Belo Monte und in Bolivien in Bezug auf Tipnis. In allen drei Fällen entschieden sich die verschiedenen Behörden für eine nationalistische Ausdrucksweise und 
das Verschweigen des Konflikts, indem sie dessen Legitimität leugneten und ihn dem ecologismo infantil (Ecuador), den Aktionen ausländischer NGOs (Brasilien) oder dem kolonialen Umweltschutz (Bolivien) zuschrieben.

Der Tipnis-Konflikt war einer der spektakulärsten Fälle. Obgleich es mehrere Episoden gab, die eine Kollision zwischen indigenen Narrativen und extraktivistischer Praxis antizipierten, erfolgte der Wendepunkt zwischen 2010 und 2011 nach dem Bau der Villa Tunari-San Ignacio Straße. Das Tipnis ist seit 1965 ein Naturschutzgebiet und seit 1990 als indigenes Territorium anerkannt, in dem amazonische Völkern leben. Die Frage war zweifellos komplex, denn der Bau der Straße war auf der einen Seite geopolitischen und territorialen Bedürfnissen unterworfen. Auf der anderen Seite war der zentrale Kritikpunkt, dass die betroffenen indigenen Völker nicht im Vorfeld konsultiert wurden. Ebenso deutete alles darauf hin, dass die Straße die Tür für zukünftige extraktivistische Unternehmungen unter anderem in Zusammenarbeit mit Brasilien öffnen würde, mit den daraus resultierenden negativen sozialen, kulturellen und ökologischen Folgen. Schließlich war die Eskalation des Konflikts zwischen indigenas und Umweltschützern auf der einen Seite und der Regierung auf der anderen so heftig, dass erstere mehrere Protestmärsche vom Tipnis bis zur Stadt La Paz durchführten. Als Reaktion auf eine Phase der staatlichen Repression bildete sich eine Koalition zwischen ländlichen Indigenen, sozialen und ökologischen Organisationen, und großen Teilen der städtischen Bevölkerung. Im Jahr 2012 berief die Regierung von Evo Morales eine Anhörung der indigenen Gemeinschaften im Tipnis ein. Nachdem diese durchgeführt worden war, wies der offizielle Bericht darauf hin, dass $80 \%$ der befragten Gemeinden dem Bau der Straße zugestimmt hatten. Ein Bericht der katholischen Kirche, der im April 2013 in Zusammenarbeit mit der Ständigen Menschenrechtskommission Boliviens durchgeführt wurde, ergab jedoch, dass die Befragung "nicht frei oder in gutem Glauben 
war, nicht den Standards der vorherigen Anhörung entsprach und mit Sonderregelungen durchgeführt wurde ${ }^{2}{ }^{2}$

Der Tipnis-Konflikt lässt zwei wichtigen Schlussfolgerungen zu, die auf bolivianischer, aber auch auf lateinamerikanischer Ebene zu lesen sind: An erster Stelle hat dieser Konflikt, wie bereits erörtert, den Diskurs der Regierung hinsichtlich deren Verständnis von Entwicklung offen gelegt, wie es sich auch im Buch des bolivianischen Vizepräsidenten Álvaro García Linera, Geopolítica de la Amazonia [Geopolitik des Amazonas] (2012), zeigt. Nach Linera gäbe es ohne »mehr Extraktivismus« keine Möglichkeit, die Sozialpolitik aufrechtzuerhalten, was das Scheitern der Regierung und die unvermeidliche Rückkehr der politischen Rechten an die Macht bedeuten würde. An zweiter Stelle wird hier deutlich, wie im Rahmen eskalierender Konflikte in solch virulenten und politisierten Kontexten, in denen der rekursive Charakter der Handlung die verschiedenen Akteure zu einem heftigen Kampf veranlasst, zwangsläufig die Möglichkeit ausgedünnt wird, eine freie, vorhergehende und fundierte Anhörung der indigenen Gemeinden gemäss dem Übereinkommen 169 der Internationalen Arbeitsorganisation (ILO) durchzuführen und stattdessen kontrovers über die Festlegung der Verfahren, Mechanismen und Themen der Anhörung gestritten wird.

Schließlich erleben wir in Fortführung der zweiten Phase seit 20132015 und bis heute eine Zuspitzung des Neo-Extraktivismus. Ein relevanter Faktor, der diese verstärkte Kontinuität erklärt, ist der Fall der Rohstoffpreise, der die lateinamerikanischen Regierungen dazu veranlasste, die Zahl der Extraktionsprojekte durch die Erweiterung der Rohstoffgrenzen weiter zu erhöhen (Moore 2013; Terán 2016). In diesem Zusammenhang waren die meisten lateinamerikanischen Regierungen nicht nur nicht auf den Fall der Preise vorbereitet (wie man an Venezuela deutlich sehen kann), sondern es konnten auch schnell direkte Abwärtstrends in Form von Handelsdefiziten (Martínez 2015) und ökonomischer Rezession (Peters 2016) beobachtet werden.

2 Siehe auch: http://cidob-bo.org/index.php?option=com_content\&view=ar ticle\&id=2014:obispos-defienden-su-informe-de-la-consulta-previa-en-eltipnis\&catid=82: noticias\&Itemid $=2$. 
Hinzu kommen der Rückgang der progressiven/populistischen Hegemonie und das Ende des progressiven Zyklus, eine Tatsache, die einen starken Einfluss auf die Neukonfiguration der regionalen politischen Landschaft haben wird, wie im späteren Verlauf diskutiert wird.

\section{Territorialität und eine neue Sprache der Inwertsetzung}

Derzeit scheint es einen impliziten Konsens zwischen verschiedenen lateinamerikanischen Analysten zu geben, dass eine der konstituierenden Dimensionen des sozialen Widerstands gegen den Extraktivismus die Verteidigung von Territorium und Territorialität ist. Sicherlich sind Gebiet und Territorialität umstrittene Konzepte, denn sie tauchen nicht nur in den Narrativen indigener Organisationen und sozio-ökologischer Bewegungen auf, sondern auch in der Debatte von Unternehmen, Planern, politischen Entscheidungsträgern, kurz gesagt, im Diskurs der politischen Macht auf ihren verschiedenen Ebenen und Skalen. Somit wurde der Begriff des Territoriums zu einer Art gesamtgesellschaftlichem Konzept, von dem ausgehend es möglich ist, die Positionierung verschiedener Konfliktparteien und noch vielmehr die Analyse der sozialen und politischen Dynamiken zu veranschaulichen.

Die Aneignung von Gebieten ist niemals nur materiell, sondern auch symbolisch (Santos 2005). Wie der brasilianische Geograph Bernardo Mançano Fernandes sagt, »leben wir zusammen in einer Welt mit Produktionsgebieten verschiedener Art, die das Resultat verschiedener sozialer Beziehungen sind und die täglich umstritten werden « (Mançano 2008). Zweifellos ist es die brasilianische kritische Geographie, die enorm zur Erweiterung und zur Reaktualisierung des Territorialbegriffs beigetragen hat, insbesondere aus einer Perspektive, die die Notwendigkeit betont, »die Territorien von unten darzustellen« (Porto 2001), das heißt eine Annäherung an Territorium und Territorialisierung im Sinne der kämpfenden sozialen Bewegungen. Für Carlos Porto Gonçalves ist die heutige Zeit mit der Renaissance vergleichbar, da wir einen Prozess der geographischen (Neu-)Gestaltung 
erleben, in dem die verschiedenen Akteure und Gesellschaftsschichten in diesen institutionellen Prozessen nicht gleichermaßen präsent sind. Territorialität entfaltet sich in einem komplexen Raum, in dem unterschiedliche Bewertungsweisen des rationalen Denkens und logischen Handelns miteinander verflochten sind. In ähnlicher Weise reflektiert der brasilianische Geograph Rogerio Haesbert (2011) über die Multiterritorialität, die für ihn das Gegenteil der Globalisierung darstellt. Tatsächlich zeichnet sich weit von einem »Ende der Territorien« eine komplexere Geographie, die Multiterritorialität mit starken rhizomatischen, nicht hierarchischen Konnotationen ab, veranschaulicht durch so genannte Netzwerk-Territorien, die von unten von subalternen Gruppen aufgebaut werden.

Im Allgemeinem gilt das Territorium sowohl in städtischen als auch ländlichen Bewegungen als Raum des Widerstands und zunehmend auch als Ort der Resignifikation und der Entstehung neuer sozialer Beziehungen. Aus der Sichtweise der sozialen Bewegungen wurde die Territorialität in ihrer materiellen Dimension oft ausschließlich als gemeinschaftliche Selbstorganisation verstanden. Dieses Verständnis traf sowohl auf die bäuerlich-indigenen als auch auf die städtischen sozialen Bewegungen zu, die durch den Kampf um Land und über Forderungen nach Grundbedürfnissen miteinander verbunden sind. Gleichwohl hatte der Disput um die Gebiete um das Jahr 2000 andere Auswirkungen, beginnend mit den neuen Bedingungen, die in für Naturressourcen strategisch gehaltenen Gegenden von der Kapitallogik übernommen wurden. In diesem Sinne können extraktive Megaprojekte, etwa der groß angelegte Bergbau für Metalle, die Ausweitung der Produktionsgrenze für Treibstoff, die Agrarwirtschaft und sogar der neoliberale Urbanismus als paradigmatische Beispiele gesehen werden, in denen die »Spannungslage der Territorialitäten« zum Tragen kommt (Porto 2001). Es wird eine dominante Vision der Territorialität entwickelt, die ausgrenzend für bestehende (oder potentiell bestehende) ist.

Im Zusammenhang des Rohstoffkonsenses lässt sich eine Zuspitzung des dominanten Territorialbegriffs beobachten. Nach dem Geographen Robert Sack (1986) wurde im Interesse des Kapitals, der Unternehmen und Regierungen eine Vision von »effizienten«, leistungs- 
basierten Territorien entworfen. Sack betrachtet diese Vorstellung als "gesellschaftlich leer«, da es hier um durch das Kapital valorisierte Waren geht. Im Namen der Ideologie des Fortschritts sind die dort angesiedelten comunidades unsichtbar, die regionalen Ökonomien wertlos und krisengeplagt, wodurch sich die Einführung neuer Entwicklungsmodelle erleichtert und die Territorien somit okkupiert werden können. Diese Prozesse der Abwertung werden in traditionell vernachlässigten Regionen begünstigt. So werden beispielsweise im argentinischen Teil Patagoniens weitläufige Gebiete als »Wüste« angesehen. Die Metapher ruft dunkle Erinnerungen hervor, da sie Ende des 19. Jahrhunderts verwendet wurde, um indigene Bevölkerungsgruppen in die Enge zu treiben, anschließend auszurotten und dadurch das, was sie in Bezug auf ihre Kulturen und Lebensräume darstellten, abzuwerten. Heute wird die Wüstenmetapher erneut von Funktionären der nationalen Regierungen und der Provinzregierungen verwendet, um beispielsweise die Notwendigkeit des groß angelegten Bergbaus, die Erweiterung der Ölgrenze durch Fracking oder die Agrarwirtschaft als einzige produktive Alternative zu propagieren.

Ähnlich ist es im riesigen Amazonas, der ein weiteres übergangenes Gebiet darstellt. Wie Porto (2017) feststellt, wird der Amazonas nicht nur als »Ressource« oder »unerschöpfliche Quelle« gesehen, sondern auch als "demographisches Vakuum «, das die dominanten Klassen der Region sich in ihrer nachgeordneten Integration in das globale Machgefüge aneignen können. Hierbei wird die geographische Komplexität der Region durch eine visión eficientista ignoriert, welche die peripheren Gebiete gleichzeitig als nutzlos und unproduktiv charakterisiert. In der lateinamerikanischen Öffentlichkeit war es zweifellos der ehemalige peruanische Präsident Alan García, der diese Vorstellung gnadenlos zum Ausdruck brachte, als er in der traditionellen, in Lima ansässigen Zeitung El Comercio den Artikel »El síndrome del perro del hortelano« veröffentlichte, in dem er seine Politik gegenüber dem Amazonas brutal antizipierte. In diesem Artikel argumentierte er, dass die AmazonasIndianer, die sich gegen die Ausbeutung der ungenutzten Gebiete wehr- 
ten, dem »Hund des Gärtners« gleichen würden. ${ }^{3}$ Für García galt das gesamte Amazonasgebiet als ungenutzt und musste durch die Ausweitung der Grenzen von Bergbau sowie der Gas- und Ölförderung zu einem effizienten und produktiven Gebiet gemacht werden. ${ }^{4}$

Zusammenfassend erleichtert die Behauptung, es gebe Regionen mit geringer Bevölkerungsdichte, die historisch von Armut und sozialer Verwundbarkeit geprägt sind und die zahlreiche Ausweitungen der Produktionsgrenzen erlebt haben, die Einrichtung eines leistungs- und produktionsorientierten sowie ausschließenden Diskurses im Namen der globalen Dynamik des Kapitals. Ob sie als sozial leere Gebiete, als Vakuum, Wüsten oder als Lebensraum der Arbeitsscheuen konzipiert sind, die Ergebnisse sind ähnlich: Die Abwertung alternativer Produktionsformen, regionaler Wirtschaftsmodelle oder, kurz gesagt, jegliche Stimmen für die Aufwertung des Lebensraums subalterner Gruppen, werden zunehmend als unvereinbar mit dem dominanten Modell gewertet.

\section{Politisch-ideologische Matrizen und ökoterritoriale Wende der Konflikte}

Bevor man über sozialen Widerstand spricht, sollte man klarstellen, dass es mindestens vier verschiedene politisch-ideologische Matrizen

3 Aus Lope de Vegas Komödie El perro del hortelano (1615) geht das Sprichwort »Ser como el perro del hortelano, que no come ni deja comer« hervor. Der fleischfressende Hund frisst das Gemüse im Garten nicht. Gleichzeitig lässt er nicht zu, dass andere Tiere es fressen.

4 Diese Ankündigungen wurden im Juni 2008 zur Realität, als die Exekutive hunderte von Gesetzesdekreten verabschiedete, darunter auch ein Paket mit elf Gesetzen, die den Amazonas betreffen. Die Dekrete, die von indigenen Organisationen und Umwelt-NCOs in »Das Regenwaldgesetz « umbenannt wurden, wurden von verschiedenen Sektoren in Frage gestellt. Schließlich fand am 5. Juni 2009 die Unterdrückung von Bagua statt, die das Leben von etwa 30 Einwohnern des Amazonasgebiets und zehn Polizisten, sowie eine unbestimmte Anzahl an Verschwundenen forderte. 
gibt, welche die wechselhaften Konfliktkonstellationen in Lateinamerika durchziehen: ${ }^{5}$ 1) die indigen-bäuerlich kommunitären, 2) die popular-bewegungsorientierten, 3) die traditionell klassenkämpferischen und 4) die etwas jüngeren autonomen Narrative.

Die bäuerlich-indigene Matrix ist im historischen Gedächtnis der indigenen Völker verankert und basiert auf der Idee des jahrhundertealten Widerstands, des Kampfes für kollektive Rechte und der Macht der Gemeinschaft. Mit Blick auf die jüngere Vergangenheit sind hier folgende Prozesse hervorzuheben: auf internationaler Ebene die Dekolonisierung und fortschreitende Anerkennung kollektiver Rechte (Aufnahme der ILO-Konvention 169 in die verschiedenen nationalen Verfassungen, später in die Allgemeine Erklärung der Rechte indigener Völker); auf regionaler Ebene die Krise des modernisierenden Entwicklungsstaates und das relative Scheitern der Integration der subalternen Landbevölkerung in eine bäuerlich-mestizische Identität; auf nationaler Ebene der Prozess der Erweiterung der ethnischen Grenzen, das heißt die immer stärkere Präsenz indigener Bevölkerung in den Städten. Diese Prozesse stehen in ideologischer Hinsicht in Verbindung mit der Krise des Marxismus und der Entstehung des Multikulturalismus als Perspektive der Identitätskonstruktion.

Unter politisch-ideologischen Matrizen verstehe ich jene Leitlinien, die das Denken über Politik und Macht sowie die Konzeption über soziale Veränderungen organisieren. Obwohl jede politisch-ideologische Matrix eine spezifische Konfiguration hat, verleihen ihnen die unterschiedlichen nationalen $\mathrm{Zu}$ sammenhänge sowie die inneren Spannungen jeweils eine Dynamik und eine besondere Historizität. Andererseits sind die politisch-ideologischen Matrizen, auf die wir uns beziehen, nicht in einem reinen Zustand, da die unterschiedlichen politischen Dynamiken diversen Verflechtungen und Konjunktionen (zwischen Indianismus und Marxismus, zwischen Indianismus und populistischer Matrix, zwischen Indianismus und autonomen Narrativen, zwischen Marxismus und Autonomie, um nur einige Beispiele zu nennen) hervorgebracht haben. Ebenso lassen sich Konflikt- und Kollisionsprozesse ausmachen, welche die Unterschiede zwischen den Matrizen in Bezug auf die Konzeption von Gesellschaft und die politische Praxis verstärken können. Siehe Svampa (2010 und 2017). 
Die zweite Matrix ist die populare beziehungsweise national-populare, die sich durch das Feld popularer Organisationen in Lateinamerika zieht. Dieses Muster ist im mittelfristigen historischen Gedächtnis etabliert, verbunden mit den fundamentalen popularen politischen Erfahrungen der 1930er und 1950er Jahre. Dabei stützt es sich auf eine dreigliedrige Affirmation der Nation: einem umverteilenden und versöhnenden Staat, einer charismatischen Führung und den organisierten Massen - dem Volk. Obwohl die populare Matrix im Allgemeinen dazu neigt, den Appell an ein radikal-nationalistisches Projekt mit dem klassischeren Modell der kontrollierten Partizipation zu verbinden, zeigt sich in der Geschichte eher letzteres. Dieses stützt sich auf gelenkte soziale und Gewerkschaftsbewegungen, die sich auf eine Führungspersönlichkeit an der Spitze eines Staatsapparates ausrichten (was zur einer Resubalternisierung und Verstaatlichung sozialer Bewegungen führt).

Drittens ist die Matrix des Klassenkampfes zu betrachten, die ein Konzept von Macht (und damit von sozialem Wandel) im Zusammenhang mit der Idee des Klassenfeindes und dem Aufbau des Sozialismus darstellt. Die besagte Matrix gründet ihre Narrative in den verschiedenen Varianten des internationalistischen Marxismus, die auf den Arbeiter als Subjekt ausgerichtet sind und in Lateinamerika in unterschiedlichen Ausdrucksformen zu finden sind (verbunden mit der kommunistischen Partei, dem Maoismus und den verschiedenen existierenden Trotzkismen). Diese auf die Arbeiterbewegung fokussierende Konzeption von Gesellschaft hat sich traditionell gegen das Verständnis der Vielfalt und sozialen Heterogenität in peripheren Gesellschaften gewendet. Es ist kein Zufall, dass der historische Fokus auf den Klassenkampf (die Fähigkeit, autonom zu handeln, das heißt als Klassenakteur) subalterner sozialer Subjekte (bäuerliche, indigene, marginale, informelle, ländliche Sektoren) in Lateinamerika die Vorstellung befördert hat, dass in den Gesellschaften der Region ein geringes Klassenbewusstsein herrsche und diese somit durch schwache Akteure oder unvollständige Subjekte gekennzeichnet seien. Daher ist die Spannung zwischen der klassenkämpferischen Matrix und dem Rest der vorhandenen Matrizen insgesamt eher manifest als latent. 
An vierter Stelle steht ein Narrativ der Autonomie, dessen Aktualisierung im Rahmen des historischen Kurzgedächtnisses erfolgt, obwohl einzelne Bestandteile bis zur anarchistischen Tradition zurückreichen. Die zentralen Elemente, aus denen sich diese Matrix zusammensetzt, sind die Bekräftigung von Autonomie, Horizontalität und Demokratie per Konsens. In diesem konkreten Fall beziehe ich mich auf ein Narrativ, weil dieses als eine Art identitäre Erzählung, der Produktion des Subjekts, aufgebaut wird, in der die persönliche Erfahrung der Akteure zählt, noch bevor sie sich einer Gemeinschaft (indigene Matrix), der Figur des Volkes (Populismus) oder einer sozialen Klasse (marxistische Matrix) zuschreiben. Historisch gesehen handelt es sich um ein Narrativ, das auf dem allgemeinen Scheitern der traditionellen Linken beruht. Deshalb nimmt es zunehmend auch zu anderen traditionellen Positionen der marxistischen Linken Abstand. Dabei ist es auch mit den Prozessen der Deinstitutionalisierung moderner Gesellschaften und neuen Individualisierungsdynamiken verbunden. Die autonome Narrative haben neue Modelle der Militanz entstehen lassen, die sowohl in Ländern des Zentrums als auch der Peripherie verbreitet sind. Die Selbstorganisation erfolgt oft in affinen Gruppen in Form von Kollektiven, die sich im Feld des Umwelt- und Kulturaktivismus sowie in den Alternativmedien und den popularen Feminismen immer weiter ausbreiten. Der Kampf gegen das Patriarchat, die künstlerische Intervention und die Volksbildung sind symbolträchtige Aspekte der neuen sozialen Mobilisierungen, die einen Epochenwechsel markieren.

Ausgehend von diesem Überblick können wir zwei Prozesse skizzieren, die über die spezifischen Merkmale des progressiven Zyklus hinausgehen. Aus institutioneller Sicht erlebten wir mit dem Entstehen progressiver Regierungen eine politische Dynamik, die - mit unterschiedlichen Nuancen und Graduierungen - zur Verstaatlichung zahlreicher sozialer Bewegungen führte. In diesem Raum wurde die populare Matrix hegemonial, wobei sie eine Spannung mit anderen politisch-ideologischen Matrizen aufbaute, sei es mit der kommunalen Form, die mit der bäuerlich-indigenen Matrix verbunden ist, oder mit dem Narrativ der Autonomie. Mit anderen Worten, während des progressiven Zyklus ist die Aktualisierung der popularen Matrix mit ei- 
nem wachsenden hegemonialen Anspruch verbunden, der auf der Ablehnung und/oder Absorption von Elementen anderer konkurrierender Matrizen - der indigen-bäuerlichen Narrative, der neuen autonomen Linken - basiert, die zu Anfang des Epochenwechsels eine wichtige Rolle gespielt hatten.

Andererseits hat die Dynamik der sozial-ökologischen Konflikte ab 2003 die Grundlage für eine gemeinsame Dimension der Bewertung von Territorialität gelegt. Dies können wir als ökoterritoriale Wende bezeichnen, die durch die Konvergenz der verschiedenen Matrizen und Narrative veranschaulicht wird, nämlich durch die innovative Verflechtung aus indigen-kommunitärer Matrix und autonomer Narrative in einem umweltpolitischen Ansatz, zu dem am Ende des Zyklus der feministische Ansatz hinzugefügt werden kann.

Folglich entstand ein gemeinsames Narrativ, das den Anspruch hat, Aufmerksamkeit darauf zu lenken, wie die gegenwärtigen sozioökologischen Kämpfe gedacht und vermittelt werden, welche sich auf die Verteidigung von Land und Territorium konzentrieren. Die ökoterritoriale Wende bezieht sich auf die Schaffung von Rahmen kollektiven Handelns, die gleichzeitig als entgegnende oder alternative Bedeutungsstrukturen und Interpretationsschemata gelten. Diese kollektiven Rahmen haben oft eine wichtige Mobilisierungswirkung, installieren neue Themen, Sprachen und Schlagwörter im Hinblick auf gesellschaftliche Debatten und lenken gleichzeitig die interaktive Dynamik auf die Produktion einer gemeinsamen Subjektivität im lateinamerikanischen Raum der Konflikte.

Die Verankerung eines alternativen Narrativs zur Bewertung der dominanten Territorialität scheint bei indigenen und bäuerlichen Organisationen unmittelbarer zu sein, sowohl aufgrund der engen Beziehung, die sie zwischen Land und Territorium in Bezug auf die Lebensgemeinschaft herstellen, als auch aufgrund der offenkundigen Reaktivierung der Matrix der indigenen Gemeinschaft in den letzten Jahrzehnten. Die ökoterritoriale Wende umfasst jedoch nicht nur die Länder, in denen es eine notorische Präsenz von indigenen Gruppen gibt, die historisch ausgegrenzt sind, sondern auch andere Länder, in denen sie durch unterschiedliche klassenübergreifende und multiethni- 
sche Erfahrungen und verschiedene Organisationsformate zum Ausdruck kommt.

\section{Sozio-ökologische Konflikte und ihre Ebenen}

Der explosionsartige Anstieg sozio-ökologischer Konflikte korrelierte mit dem, was der renommierte mexikanische Essayist Enrique Leff »Ökologisierung der Kämpfe der Indigenen und Bauern und Notstand der lateinamerikanischen Denkweise über Umwelt « nannte (Leff 2004). Darüber hinaus ist das Szenario nicht nur durch die Kämpfe der Indigenen und Bauern gekennzeichnet. Stattdessen entstehen neue sozio-ökologische Bewegungen sowohl in den ländlichen als auch städtischen Regionen (kleiner und mittlerer Größe), deren Mitglieder verschiedenen sozialen Klassen angehören und die sich durch ihre versammlungsähnliche Organisation und ihre Wirkung als Antagonist auszeichnen. ${ }^{6}$ Gleichzeitig spielen in diesem neuen sozialen Gefüge

6 Es ist wichtig hervorzuheben, dass das Konzept der sozialen Bewegung scheinbar in eine Krise geraten ist. Einige Autoren, darunter Silvia Rivera Cusicanqui, schlagen vor, das Konzept komplett ad acta zu legen, da es von progressistischen Persönlichkeiten zu stark instrumentalisiert wurde (zunächst in Bolivien, in dessen Fall oft von der »Regierung der sozialen Bewegungen« gesprochen wird). Andere Stimmen wie Raúl Zibechi schlagen vor, den Begriff durch das Konzept der »Cesellschaft in Bewegung « zu ersetzen. Dieses steht zwar noch weit am Anfang, hat aber analytisches Potential. Einige weitere bevorzugen die Bezeichnung »antagonistische Bewegungen«, darunter Massimo Modonesi (2016). Aus meiner Sicht gilt es einerseits, soziale Bewegungen im engeren Sinne zu fassen. Diese Lesart, die in Lateinamerika vorherrscht, fokussiert auf soziale Bewegungen als Akteure, die die gegebene Herrschaft in Frage stellen. Diese theoretische Definition impliziert die Möglichkeit, soziale Bewegungen als potentiell antagonistische Subjekte im Rahmen eines Herrschaftssystems zu betrachten. Andererseits schlage ich vor, von sozialen Bewegungen im weiteren Sinne zu sprechen: Hierbei ist eine Art kollektives Handeln der Akteure gemeint, die nicht in der Macht stehen, mit dem Ziel, ein Element des etablierten Gesellschaftssystems durch eine umstrittene Aktion mit einer gewissen organisatorischen Kontinuität zu verändern. Das Thema wird genauer in Svampa (2017) behandelt. 
verschiedene kulturelle Kollektive, bestimmte Umwelt-NGOs (mit der Philosophie einer sozialen Bewegung), intellektuelle Persönlichkeiten und Experten, die das Handeln sozialer Organisationen und Bewegungen begleiten - und dieses sogar unmittelbar beeinflussen - eine nicht unbedeutende Rolle. Wie so oft in anderen Zusammenhängen der Bekämpfung sind die Hauptakteure dieser Organisationsdynamiken junge Erwachsene, viele von ihnen Frauen, deren Rolle sowohl in den großen Organisationsstrukturen als auch in den unterstützenden kleinen Gruppen maßgeblich ist.

Die Schnittpunkte und Verbindungen zwischen den Organisationen führten $\mathrm{zu}$ zahlreichen Koordinationsbündnissen wie Via Campesina oder - auf einer anderen Ebene - thematischen Foren, etwa zur Verteidigung des Wassers, der Verteidigung natürlicher Ressourcen und gegen Fracking. Weiterhin entstanden Plattformen für gemeinsame Aktionen, zum Beispiel gegen die Freihandelszone der Amerikas (FTAA), gegen die Megaprojekte der Infrastrukturinitiative zur globalen Integration Südamerikas (IIRSA) und aktuell gegen die Transpazifische Partnerschaft (TTP). In dieser Hinsicht stellt die Kommunikation zwischen verschiedenen Akteuren eine Innovation dar. Durch sie wird der Dialog zwischen Wissen und Disziplinen gefördert, der sich durch die Aufwertung des lokalen, traditionellen Wissens sowie durch die Erarbeitung von Expertenwissen unabhängig von den dominanten Diskursen auszeichnet. Das Thema ist nicht unbedeutend, denn ausgehend von dieser Artikulation erarbeiten die Organisationen und Bewegungen gemeinsame Lösungen, erweitern die Diskussionsplattform über lokale und nationale Probleme hinaus und gestalten ihre Strategien vielfältig. Hierbei wird die Mobilisierung als Grundlage etwa mit dem Posten in sozialen Netzwerken und der Generierung und Anwendung verschiedener fachlicher und praktischer Hilfsmittel verbunden (z.B. kollektive Schutzmaßnahmen, neue Verordnungen, Forderung nach öffentlichem Beistand und nach Gesetzen für den Umweltschutz und die Rechte indigener Völker).

Von allen mineralgewinnenden Aktivitäten wird der in großem Maß angelegte Bergbau ohne Zweifel am meisten kritisiert. Derzeit gibt es kein lateinamerikanisches Land mit Bergbauprojekten, in dem keine 
sozialen Konflikte zwischen Unternehmen, der Regierung und den Gemeinden herrschen: Mexiko, Guatemala, El Salvador, Honduras, Costa Rica, Panama, Ecuador, Peru, Kolumbien, Brasilien, Argentinien und Chile. Es gibt mehrere fest verankerte Zusammenschlüsse, die sich dem Thema Bergbau widmen, darunter das 1991 gegründete Observatorio Latinoamericano de Conflictos Ambientales (OLCA) mit Hauptsitz in Chile sowie das Observatorio de Conflictos Mineros de América Latina (OCMAL), das seit 1997 als Sprachrohr für mehr als 40 Organisationen wirkt, darunter auch Acción Ecológica aus Ecuador. So gab es laut OCMAL im Jahr 2010 genau 120 solcher Bergbaukonflikte, von denen 150 Gemeinden betroffen waren; 2012 waren es 161 Konflikte, darunter 173 Projekte und 212 betroffene Gemeinden. Im Februar 2014 lag die Zahl der Konflikte bei 198, wobei 279 Gemeinden betroffen und 207 Projekte beteiligt waren. Im Januar 2017 gab es 217 Konflikte, an denen 227 Projekte und 331 Gemeinden beteiligt waren. Die Länder mit der höchsten Anzahl an Konflikten sind Peru (39), Mexiko (37), Chile (36), Argentinien (26), Brasilien (20), Kolumbien (14) und Ecuador (7). Zudem finden sechs Konflikte in Grenzgebieten statt (OCMAL). Laut dem Environmental Justice Atlas (EJAtlas) erfolgte ein Anstieg der Konflikte ab 1997 und war zwischen 2006 und 2008 besonders deutlich. Die Zahlen von OCMAL zeigen einen Anstieg zu ähnlichen Zeitpunkten (Villegas 2014, 10-11). ${ }^{7}$

Es ist unmöglich, alle in Lateinamerika existierenden sozioökologischen Konflikte offenzulegen. Das gleiche gilt für eine etwaige Liste der selbstorganisierten umweltbezogenen Netzwerke, sowohl auf nationaler als auch auf regionaler Ebene. Ohne Anspruch auf Vollständigkeit und zur Veranschaulichung wird im Folgenden kurz auf einige Konflikte und Netzwerke in Ländern wie Peru, Bolivien,

7 Der EJAtlas ist ein Projekt, an dem ein internationales Expertenteam von 23 Universitäten und Umweltgerechtigkeitsorganisationen aus 18 Ländern beteiligt ist. Das Projekt wird von Forschenden des Instituts für Umweltwissenschaften und -technologie (Institut de Ciència i Tegnologia Ambientals, ICTA) der Universidad Autónoma de Barcelona unter der Leitung des Umweltökonomen Joan Martínez Alier koordiniert. Cleichzeitig handelt es sich um eine kollektive Initiative, an der die Zivilgesellschaft teilhat. 
Nicaragua, Ecuador, Kolumbien, Mexiko und Argentinien eingegangen. ${ }^{8}$ In Peru, einem durch den groß angelegten Bergbau geprägten Land, repräsentierten 2013 laut der Defensoría del Pueblo (dt. etwa »Büro des Bürgerbeauftragten«) insgesamt 120 mit dem Bergbau zusammenhängende Konflikte $48 \%$ der sozialen Konflikte. ${ }^{9} 2016$ stieg diese Zahl auf $68 \%$ an, womit die Konflikte, die die peruanischen Behörden im gesamten Land registriert hatten, 220 betrug. Von diesen waren 150 mit der Vergabe von Bergbauprojekten verbunden. ${ }^{10}$ Unter den Pionierorganisationen auf kontinentalem Niveau im Kampf gegen den Megabergbau sticht die Confederación Nacional de Comunidades Afectadas por la Minería (Conacami), gegründet 1999, heraus. Eine weitere wichtige Organisation ist die Grupo de Formación e Intervención para el Desarrollo Sostenible (Grufides) in Cajamarca, die eine große Reichweite in Sachen Intervention und Bekämpfung hat und dessen Vorsitzender, der Ex-Priester und Soziologe Marco Arana, 2009 die Partei Tierra y Libertad gründete. ${ }^{11}$ Inzwischen hat die Conacami keine territoriale Präsenz mehr und verfügt nicht mehr über die gleichen

8 Es existieren zahlreiche Veröffentlichungen zu sozialökologischen Konflikten auf nationalem Niveau, die unmittelbar mit dem Extraktivismus in Lateinamerika zu tun haben. Für den Kontext Peru sind de Echave (2009) sowie Hoetmer, Castro, Daza und de Echave (2013) empfehlenswert. Letzterer vermittelt kontranarratives Expertenwissen im Hinblick auf soziale Bewegungen gegen den Megabergbau. Für den Fall Bolivien siehe Veröffentlichungen des Centro de Información y Documentación de Bolivia (2014); für Kolumbien Roa und Navas (2014) und Archilla (2015); für Mexiko siehe Composto und Navarro (2011) und Navarro (2015), ebenfalls Delgado (2010) und Lemus (2018). Für eine Kartographie der ökologischen Konflikte in Argentinien siehe Merlinsky (2016), Giarranca und Teubal (2013), Svampa und Viale (2014), Sola und Bottaro (2013) und Machado (2013; 2014).

9 Siehe www.defensoria.gob.pe/blog/mineria-y-conflictos/.

10 Contralínea (2016). »Perú: 150 conflictos mineros«. Zugriff: 12. Januar 2017. www.contralinea.com.mx/archivo-revista/index.php/2016/09/16/peru-150conflictos-mineros/.

11 Die Partei, die sich später in Tierray Dignidad (dt. Land und Würde) umbenannte, war Teil des linken Wahlbündnisses Frente Amplio de Izquierda, dessen Spitzenkandidatin Verónika Mendoza bei den Präsidentschaftswahlen 2016 den dritten Platz belegte. 
Kapazitäten, die sie zwischen 2008 und 2009 hatte. Angesichts dessen haben sich verstärkt andere lokal-organisatorische Strukturen herausgebildet, wie die rondas campesinas, eine Art von Bauernpatrouille, deren Rolle immer bedeutender im Kampf gegen den Megabergbau wird (Hoetmer, Castro, Daza und de Echave 2013, 268).

In Bolivien reicht die extraktivistische Welle vom Bergbau über die Erdöl- und Erdgasförderung und den Vormarsch der Agrarindustrie bis neuerdings zu einer Reihe von Energieprojekten, die in der so genannten Agenda Patriótica 2025 und dem neuen Nationalen Entwicklungsplan enthalten sind. Dieser sieht den Bau mehrerer großer Dämme und eines Atomkraftwerks in El Alto vor. Wie bereits zuvor erwähnt entstand aus dem Bau einer Landstraße 2011 der Konflikt von Tipnis, der einen Wendepunkt markierte. Die Verteidigung des Extraktivismus wurde vom Vizepräsidenten Álvaro Garcia Linera angeführt, der im Jahr 2015 vier bolivianischen NGOs (Centro de Documentacion e Información Bolivia [Cedib], Terra, CEDLA und Milenio) mit der Ausweisung drohte und ihnen unterstellte, sie würden »die Interessen der internationalen Rechten « vertreten. ${ }^{12}$ Wie viele andere Organisationen recherchieren diese über den Neo-Extraktivismus und über die Ausweitung der Grenzen für die Agrarindustrie. 2016 verabschiedete die Regierung ein neues Gesetz, das die Vereinigungsfreiheit einschränken soll und kritische NGOs der Gefahr der Schließung aussetzt, sofern diese sich nicht an die Agenda Patriótica 2025 und den Nationalen Entwicklungsplan halten. 2017 spitzte sich die Situation der Bedrohungen und der Verfolgung von Cedib so $\mathrm{zu}$, dass es der Organisation fast unmöglich war, weiterzuarbeiten.

Einer der besorgniserregendsten Fälle ist Ecuador. Obwohl die dortige Verfassung die Rechte der Natur festschreibt, lautete die Antwort der Correa-Regierung auf die sozio-ökologischen Konflikte gleich Kriminalisierung und Verrechtlichung der Proteste. Es kam in Strafprozessen gegen Sprecher indigener Organisationen zu Gefäng-

12 Vgl. Öffentlicher Brief von Intellektuellen an den Vizepräsidenten Carcía Linera unter www.eldeber.com.bo/intelectuales-piden-garcia-linera-respete.html. 
nisstrafen von bis zu zehn Jahren. ${ }^{13}$ Darüber hinaus erkannte man einigen NGOs ihren Rechtsstatus ab und wies sie aus, darunter die Fundación Pachamama (2013). Neben der Schikanierung erfolgten Drohungen, die renommierte NGO Acción Ecológica aufzulösen (sowohl 2009 als auch 2016). Darüber hinaus wurde 2014 und 2015 gewarnt, Visa könnten aufgehoben sowie ausländische Berater, die mit Anführern aus der Umweltszene in Verbindung stehen, ausgewiesen werden. Ebenso machte die ecuadorianische Regierung von Gesetzeslücken Gebrauch, um die von der Bürgerbewegung Yasunidos vorgeschlagene Forderung nach einer Volksinitiative zu entkräften, nachdem sie einseitig beschlossen hatte, das Stillhalteabkommen bezüglich des Parque Yasuní zu brechen und mit der Ölförderung zu beginnen. Trotz des starken Widerstandes gegen den Megabergbau - Ecuador hat keine Tradition des großangelegten Bergbaus - ist die Regierung ab 2013 durch die Militarisierung der Gebiete vorangeschritten. Unter diesen Gebieten ist Intag, das als Bollwerk im Kampf gegen solche Aktivitäten gilt. Die Bevölkerung vertrieb dort mehrere Bergbauunternehmen und setzte stattdessen auf alternative Entwicklungsmöglichkeiten. Hinzu kommt, dass chinesische Unternehmen, die die Investments in den Bergbau im Land anführen, der unzumutbaren Arbeitsbedingungen bezichtigt wurden. ${ }^{14}$ Laut Acción Ecológica wurden bereits 2012 chinesische Unternehmen, die mit dem Bergbauprojekt Mirador

13 Siehe den Bericht der Fédération internationale des ligues des droits de l'Homme (FIDH), der Fälle der Kriminalisierung von Menschenrechtsverteidigern in Lateinamerika erfasst; darunter sind auch Fälle der Kriminalisierung der Intag und der indigenen Völker der Shuar-Föderation (2015). Nachzulesen unter https:// www.fidh.org/IMC/pdf/criminalisationobsangocto2015bassdef.pdf.

14 Darüber hinaus genießen chinesische Unternehmen viele Vorteile, da sie »Projekte durchführen, die über chinesische Kredite und zu hohe Zinsen finanziert werden, und außerdem ist die Bezahlung in der Regel von den von ihnen gelieferten Vorleistungen und der Beschäftigung chinesischer Arbeitskräfte abhängig«. Siehe http://catapa.be/es/noticias/el-cuento-de-la-miner \%C3ADa-chinaen-ecuador.

Für mehr Informationen über den Bergbau und zur Präsenz chinesischer Unternehmen in Ecuador siehe Chicaiza (2014). 
in Verbindung stehen, wegen Nichteinhaltung von Arbeitsstandards, Misshandlungen, ungerechten Löhnen und Unfällen durch die ShuarGemeinschaft angeprangert. 2016 entstanden neue Konflikte, als einige Shuar-Indigene ein Bergbaugebiet im Amazonas besetzten. Die Ansiedlung des chinesischen Unternehmens erfolgte ohne vorherigen Dialog und durch eine Militarisierung der Gebiete. Im Dezember desselben Jahres eskalierte der Konflikt in Folge der Forderungen der Shuar so stark, dass es einen Toten und mehrere Verletzte gab. Die Reaktion des damaligen Präsidenten Correa bestand darin, den nationalen Ausnahmezustand auszurufen, die Shuar als "paramilitärische und halbkriminelle Gruppen « $\mathrm{zu}$ bezeichnen und die Auflösung von Acción Ecológica bekanntzugeben. Angesichts der nationalen und internationalen Solidaritätsbekundungen lehnte das ecuadorianische Umweltministerium den Auflösungsantrag des Innenministeriums am Ende $\mathrm{ab}$.

Ebenso hingen in Kolumbien zwischen 2001 und 2011 rund $25 \%$ der Konflikte mit dem Abbau von Öl, Gold und Kohle zusammen (Roa und Navas 2014, 35). Während seiner Präsidentschaftskampagne im Jahr 2010 lancierte Juan Manuel Santos den Slogan »Colombia, la locomotora minera-energética « (dt. etwa »Kolumbien, die Lokomotive der Kohleenergie«). Eines der Bergbauprojekte, die auf starken Widerstand gestoßen sind, heißt La Colosa. Das Projekt wurde von der Firma Anglogold Ashanti betrieben, und La Colosa wäre im Fall der endgültigen Durchsetzung die weltweit fünftgrößte Goldmine gewesen. Betroffen gewesen wären zahlreiche Städte im Departmento Tolima, das als Kornkammer Kolumbiens gilt. Dort wurden Umweltkomitees zur Verteidigung der Lebensgrundlagen geschaffen, die öffentliche Anhörungen forderten. Nach einer ersten Anhörung in Piedras begannen die Umweltkomitees 2013 damit, Gespräche in Cajamarca und Ibagué zu organisieren, wobei sie auf ernstzunehmende rechtliche und wirtschaftliche Hindernisse stießen. Schließlich konnte im April 2017 eine öffentliche Anhörung in Cajamarca stattfinden, welche auf negative Aussichten für die Umsetzung von La Colosa hindeutete. 
Im selben Jahr entschied Anglogold Ashanti angesichts der fehlenden sozialen Ermächtigung, alle Projektaktivitäten einzustellen. ${ }^{15}$

Trotzdem ist der großangelegte Bergbau nicht der einzige Konfliktherd im Kontext des Extraktivismus in Kolumbien. Man stößt hier auch auf den Plan Maestro de Aprovechamiento del Río Magdalena, zu Deutsch »Masterplan für die Nutzung des Magdalena-Flusses. Er gilt als wichtigster Fluss des Landes, ist 1500 Kilometer lang und entspringt in der Zentralkordillere. Die Vergabe des Flusses ist im Sinne der Politik der IIRSA und weit davon entfernt, die ökologischen und sozialen Bedingungen im Gebiet zu verbessern. Stattdessen ist das Ziel, ihn in eine große Wasserstraße umzuwandeln, durch die auf großen Containerschiffen Kohle, Öl und Palmblätter für den Export transportiert werden sollen. Ein weiteres Vorhaben ist die Umwandlung in einen großen Energieerzeuger durch das Errichten mehrerer Staudämme, von denen viele den Bergbauprojekten als Stromlieferant dienen würden. Dieser umfangreiche Privatisierungsplan des Magdalena-Flusses (der im Übrigen von einem chinesischen Unternehmen gesteuert wird) hat eine Bewegung ausgelöst, die den Namen El Río de la Vida (dt. Fluss des Lebens) trägt.

In Mexiko wurde 2008 die Asamblea Nacional de Afectados Ambientales (ANAA) gegen Megabergbau, Staudämme, Urbanisierung des Urwalds und gegen riesige agroindustrielle Farmen ins Leben gerufen. Es gibt hierbei sinnbildliche Erfahrungen wie die des Consejo de Ejidos y Comunidades Opositoras a la Presa La Perota (CECOP) ${ }^{16}$, der zehn Jahre lang den Kampf der indigenen Bauern im Bundesstaat Guerrero unter dem Slogan »Wir sind die Hüter des Wassers« einte (Navarro

15 In den vergangenen Jahren fanden einige Lockerungen bei den Umweltkontrollen statt. Im gleichen Zeitraum hat Anglogold Ashanti, ein südafrikanisches Unternehmen, das mehrheitlich in den Händen US-amerikanischer und britischer Aktionäre liegt, Bergbaulizenzen aufgekauft, was in der kolumbianischen Gesellschaft so gut wie unbemerkt blieb.

16 Das ejido ist eine Besitzform, die durch gemeinnützigen, öffentlichen Grundbesitz und teils individuelle Nutzung gekennzeichnet ist. Die deutsche Bezeichnung für CECOP würde in etwa »Rat der Ejidos und Cemeinden gegen den Staudamm La Perota«lauten. 
2015, 141). Ein weiteres wichtiges Beispiel ist die Geschichte des Frente Amplio Opositor (FAO) gegen das Bergbauunternehmen San Xavier. Hierbei entstand ein Raum für zahlreiche öffentliche Aktivitäten, Informationsrunden, runde Tische, und es kam zu Anhörungen und Rechtsstreitigkeiten. Diese hatten ihren Höhepunkt im Jahr 2006, als das Unternehmen das die Infrastruktur zur Förderung der Erzlagerstätte errichtete (die ursprünglich den Abriss der ganzen Stadt vorsah) (Composto und Navarro 2011, 51).

Auf der anderen Seite gibt es in Nicaragua eines der gewagtesten und umstrittensten Megaprojekte der Region. Der Interozeanische Kanal Nicaraguas, der dreimal so groß wie der Panamakanal werden soll, wurde beim chinesischen Unternehmen Hong Kong Nicaragua Canal Development (HKND) in Auftrag gegeben. Im November 2015 wurde der Beginn der Bauarbeiten gestoppt. Der Grund waren Bauernproteste und offene Fragen zu den Umweltauswirkungen in Folge einer von internationalen Experten durchgeführten Studie, die von der Academia de Ciencias de Nicaragua in Auftrag gegeben worden war (2015). In der Folge entstand der Consejo Nacional por la Defensa de la Tierra, el Lago y la Soberanía Nacional (dt. Nationaler Rat zur Verteidigung des Landes, des Sees und der nationalen Souveränität). Der erste Protest der betroffenen Gemeinden fand 2014 statt. Ende 2016 wurde ein Bauernmarsch gegen das Kanalprojekt, das bis nach Managua reichen sollte, durch Polizei und Militär unterdrückt, wobei zahlreiche Menschen angeschossen oder auch verhaftet wurden. Trotzdem konnten die Bauarbeiten bisher nicht begonnen werden. Sie würden unzählige Gemeinden betreffen und hätten schwerwiegende Auswirkungen auf den Nicaraguasee, der die größte Süßwasserreserve der Region ist.

Zum Abschluss sollen die Versammlungen zur Verteidigung des Wassers in Argentinien thematisiert werden, die sich 2006 in Unión de Asambleas Ciudadanas (UAC) zusammenschlossen. Ihre Wurzeln haben diese Versammlungen im Kampf gegen Megabergbau und in geringerem Maße - in der Kritik am Modell der Agrarindustrie. Die UAC tritt in Form einer Versammlung auf und trifft sich dreimal jährlich mit dem Ziel, gemeinsame Strategien des Widerstands gegen das Vordringen des Bergbaumodells in zwölf Provinzen zu entwickeln. 
Hierbei geht es auch darum, die insgesamt sieben Provinzgesetze zu verteidigen, die Megamining in Argentinien verbieten. Das Herzstück des Agrarkapitalismus in Argentinien ist im agroindustriellen Kontext der erweiterte Anbau transgener Sojabohnen. Der Widerstand in der dortigen Bevölkerung war trotz der Vorreiterrolle der Madres del Barrio Ituzaingó ${ }^{17}$ in der Provinz Córdoba nur mit Mühe sichtbar zu machen. Seit 2007 gibt es die Kampagne »Paren de fumigar« (dt. Schluss mit den Pestiziden), die vom Centro de Protección de la Naturaleza (Cepronat) in Santa Fe, der Grupo de Reflexión Rural (GRR) und weiteren Gruppen gefördert wird (Melón 2014, 79). In diesem Prozess der Sichtbarmachung war die Rolle von Ärzten und Wissenschaftlern wie Andrés Carrasco und anderen Fachleuten, die das Red de Médicos de Pueblos Fumigados (dt. Ärztenetzwerk für pestizidbelastete Bevölkerungen) ins Leben gerufen haben, von großer Bedeutung. ${ }^{18}$

17 Madres del Barrio Ituzaingó ist eine ortsansässige zivile Widerstandsgruppe, die vor allem auf die gesundheitlichen Auswirkungen des Anbaus transgener Sojabohnen in der Bevölkerung aufmerksam macht.

18 Im Jahr 2009 wurde eine Studie des Wissenschaftlers Dr. Andrés Carrasco, Professor für Embryologie und leitender Forscher und Direktor des Consejo Nacional de Investigaciones Científicas y Técnicas (Conicet) sowie Leiter des Labors für molekulare Embryologie der Medizinischen Fakultät der Universität Buenos Aires publik. Die Studie untersuchte die schädlichen Auswirkungen agrochemischen Glyphosats auf Embryonen. Es wurde nachgewiesen, dass schon bei einer bis zu 1500mal geringeren Dosis als der auf argentinischen Feldern üblichen Glyphosatdosierung Darm- und Herzerkrankungen, Fehlbildungen und neuronale Veränderungen entstehen. Die Diffamierungskampagne gegen Carrasco führte unter anderem zu anonymen Drohungen gegen den Autor, medialen und institutionellen Schmutzkampagnen sowie starkem politischem Druck. Dies führte zu einer von mehr als 300 nationalen und internationalen Forschern und Kollegen unterzeichneten Unterstützungserklärung zur Verteidigung der Forschungsfreiheit und der öffentlichen Ethik. Die Schikane und Intoleranz hat seither eher noch zugenommen, da die unabhängige wissenschaftliche Forschung zu Agrochemikalien und genetisch verändertem Genmaterial zunehmend aussagekräftigere Ergebnisse hervorbringt. 


\section{Kapitel 3: Reichweite der ökoterritorialen Wende}

In diesem Kapitel sollen die mit der ökoterritorialen Wende verbundenen Themen beleuchtet werden. Zunächst einmal verstehe ich unter der ökoterritorialen Wende eher einen Trend, was bedeutet, dass es über das Allgemeine hinaus notwendig ist, die Prozesse des Widerstandes von Fall zu Fall zu betrachten. Darüber hinaus werde ich die Dilemmata in der Anerkennung der Rechte der Indigenen sowie die zunehmende Bedeutung der Rolle der Frauen hervorheben und infolgedessen die Entstehung neuer Formen des feminismo popular in der Region hervorheben.

\section{Aspekte der ökoterritorialen Wende}

In der ökoterritorialen Wende existieren bedeutende Kontaktpunkte zu dem, was von den Beteiligten selbst als »Bewegung für die Umweltgerechtigkeit « bezeichnet wird. Sie hatte ihren Ursprung in den 1980er Jahren in den schwarzen Gemeinden der Vereinigten Staaten. Beteiligte in anderen Ländern, die sich dem Trend anschließen, verstehen den Begriff der Umweltgerechtigkeit als »das Recht auf eine sichere, gesunde und produktive Umwelt für alle, wobei die Umwelt in ihrer Gesamtheit, einschließlich der ökologischen, physischen, konstruierten, sozialen, politischen, ästhetischen und wirtschaftlichen Dimensionen zu betrachten ist«. Dieser Ansatz, der die Aufmerksamkeit auf die soziale Ungleichheit bei Umweltbelastungen, den Mangel an Partizipation und 
Demokratie, ökologischen Rassismus gegenüber den Ureinwohnern sowie auf die Ungerechtigkeit zwischen den Geschlechtern und die ökologische Schuld lenkt, ist der Ursprung für das Entstehen verschiedener Umweltgerechtigkeitsnetzwerke in Lateinamerika. Beispiele sind OLCA aus Chile und das Red de Justicia Ambiental in Brasilien. ${ }^{1}$

Eines der am stärksten mobilisierenden Konzepte der ökoterritorialen Wende ist das so genannte buen vivir (dt. gutes Leben), auf Kichwa sumak kawsay, auf Aymara suma qamaña, auf Guarani ñandareko. Das buen vivir erscheint als utopischer Ausblick und hat seinen Ursprung in vielfältigen indigenen Weltanschauungen. Der Versuch, es einem einzigen, bestimmten Volk oder einer Kultur zuzuschreiben oder in ein neues binäres Schema einzuordnen, wäre schlichtweg falsch, da ein solches Schema letztlich mit den bereits seit dem kolonialen Diskurs etablierten Dichotomien einhergeht (Lang und Mokrani 2012). Das Konzept des buen vivir stellt neue Formen der Beziehung des Menschen zur Natur und seinen Mitmenschen auf. Es fordert daher den Übergang von einem anthropozentrischen Paradigma zu einem beziehungsbasierten Paradigma. In den Leitlinien dieses neuen zivilisatorischen Paradigmas stechen folgende Gedanken hervor: die Abkehr von der Idee der Entwicklung als unbegrenztes wirtschaftliches Wachstum, die Hinwendung zu einer solidarischen und nachhaltigen Wirtschaft und zur Anerkennung anderer Ansätze der Bewertung von Arbeit und Gütern jenseits des Profitdenkens, kurz gesagt: es geht um eine Vertiefung der Demokratie.

Einer der zentralen Standpunkte des buen vivir ist die Verbindung des Menschen mit der Natur, der zugleich zentraler Bestandteil derselben ist. Auf diese Weise vereint das buen vivir eine neue Wertschätzung der Natur aus ökologischen, religiösen, ästhetischen oder kulturellen Blickwinkeln. Der Vorschlag lautet, dass das wirtschaftliche Wachstum ausgesetzt werden sollte, um das Leben zu erhalten. Eine solche Vision basiert daher auf der Anerkennung der Rechte der Natur, was nicht mit einem unberührten Zustand der Natur zu verwechseln ist. Vielmehr wird auf die Respektierung ihrer integralen Existenz und die Erhaltung

1 Für mehr Informationen hierzu siehe www.olca.cl/oca/justicia/justiciao2.htm und www.justiciaambiental.org.br/justiciaambiental. 
der regenerativen Lebenszyklen, ihrer Struktur und ihrer Funktionen und auf Evolutionsprozesse sowie auf die Verteidigung der Lebenssysteme Wert gelegt (Gudynas 2009a). Die Rechte der Natur stellen einen zivilisatorischen Wandel dar und werden zu einer zukunftsweisenden Antwort auf die aktuelle gesellschaftliche Krise. Im Einklang mit dem Gedanken des buen vivir oder sumak kawsay geht es um den Aufbau einer Gesellschaft, die auf der harmonischen Beziehung zwischen dem Menschen und der Natur beruht. Wenn also die Entwicklungsstrategie darauf abzielt, das Leben auf der Erde zu »verwestlichen«, schützt das buen vivir die Vielfalt und schätzt und respektiert das "Andere« (Acosta 2010). Schließlich sei daran erinnert, dass die Debatte über die Rechte der Natur unter anderem durch die neue Verfassung Ecuadors auf die politische Agenda gesetzt wurde. In Ecuador ist die Natur eigenständiges Rechtssubjekt, was in der Verfassung genauer als »das Recht auf volle Achtung ihrer Existenz, sowie auf Erhaltung und Erneuerung ihrer Lebenszyklen, ihrer Struktur, ihrer Funktionen und ihrer evolutiven Prozesse« definiert wird (Artikel 71). Trotzdem wird diese aus Lateinamerika kommende Tendenz nicht weltweit geteilt und stellt auch hier nicht die Norm dar.

Ein weiteres Themengebiet der ökoterritorialen Wende bezieht sich auf die Konzeption von Naturressourcen als Gemeingut (commons). Diese ist eine Schlüsselfrage bei der Suche nach einem alternativen Paradigma sowohl im Globalen Norden als auch im Süden. In Lateinamerika kann der Begriff des Gemeinsamen zwei Bedeutungen annehmen. Auf der einen Seite existiert ein Widerstand gegen die verschiedenen Formen des Neo-Extraktivismus und gegen die schrittweise Ausweitung der Kommerzialisierung. Die Verteidigung der natürlichen Gemeingüter wendet sich gegen das land grabbing, die Privatisierung von Saatgut und die Über-Ausbeutung der Natur. Auf der anderen Seite öffnet der Begriff des Gemeinguts auch eine neue Perspektive auf die sozialen Beziehungen: Diese erlangen eine hohe Relevanz, indem sie Räume und Formen der sozialen Kooperation, der gemeinschaftlichen Nutzung von Gütern und des gemeinsamen Wohlstands kreieren. Dies wurde von dem Mexikaner Gustavo Esteva (2007) als »ámbitos de comunidad« (dt. »Gemeinschaftlicher Wirkungsbereich«) bezeichnet. 
Es ist wichtig zu betonen, dass die peripheren Gebiete historisch gesehen Orte der Solidarität waren. Außerhalb der formellen Wirtschaft und in Abwesenheit des Staates musste sich ein Großteil der popularen Sektoren der Bevölkerung anhand von selbstverwalteten Kooperationsstrukturen entwickeln und reproduzieren. In den Anden ist das Fortbestehen des Gemeinschaftlichen gewöhnlich ausschlaggebend für die Aufrechterhaltung der Kooperations- und Interdependenznetzwerke. In städtischen Kontexten, die durch ungleiche Modernisierung gekennzeichnet sind, herrscht ein Gefühl der Entwurzelung, und es ist notwendig, ein neues Klima der Solidarität aufzubauen. Angesichts des Vormarschs der Einfriedung und der Beschlagnahmung der Commons sowie vor dem Hintergrund der allgemein kapitalistischen Verdinglichung im Zusammenhang mit der Enteignung und Kommodifizierung des Lebens manifestieren sich in der Gegenwart neue Widerstände. So entstehen Räume für das Gemeinschaftliche und Formen der Gemeinschaftsfähigkeit, etwa Flächen für kollektive Experimente, die die Produktion und Reproduktion der gemeinsamen Güter jenseits von Staat und Markt neu bestimmen.

Die verschiedenen Berührungspunkte der ökoterritorialen Wende deuten hin auf die Entstehung einer neuen Art des Kämpfens, auf die Entstehung einer neuen Sprache des Widerstandes. Diese Tendenzen finden in Lateinamerika eine starke Resonanz und bilden ein neues, gemeinsames Sinngefüge. Diese Entwicklung verschafft indigenen Widerständen und neuen ökoterritorialen und feministischen Kämpfen Ausdruck, die in klarer Opposition zum dominanten Modell auf die Ausweitung der Rechtsgrenzen hinweisen. Ob es sich um Stimmen zur Verteidigung von Territorien und Gemeingütern, für die Menschenrechte, für die Naturrechte oder das buen vivir handelt, die Forderung weist auf eine Demokratisierung der Entscheidungen hin, aber noch viel eher auf das Recht der Bevölkerung, sich entschlossen Projekten entgegenzustellen, die die Lebensbedingungen der Schwächsten ernsthaft beeinträchtigen und die Zukunft kommender Generationen gefährden.

Trotz der globalen Auswirkungen dieser Entwicklung im Bereich des Widerstandes gegen die neoliberale Globalisierung muss berück- 
sichtigt werden, dass die neuen Sinnstrukturen noch nicht zu gesamtgesellschaftlichen Debatten geworden sind. Trotzdem haben sie (nicht ohne Hindernisse) beachtliche Fortschritte dabei gemacht, verschiedene Themen auf die öffentliche und politische Agenda zu setzen. Dahingehend wäre es ein Fehler, diese kollektiven Rahmenbedingungen angesichts der Heterogenität der Organisationen und Traditionen des Widerstands so $\mathrm{zu}$ interpretieren, als seien sie eine einzige Stimme oder als würden sie die verschiedenartigen Erfahrungswerte übergehen. In Wirklichkeit ist es notwendig, die ökoterritoriale Wende als eine Tendenz zu lesen, die die Widerstände durchzieht. Parallel dazu trugen die symbolträchtigen sozio-ökologischen Konflikte (insbesondere während der zweiten Phasen des Rohstoffkonsenses) zur Sichtbarmachung bei und auch dazu, die Debatte auf die Umweltfrage auszudehnen. Trotzdem neigen die meisten Regierungen und nicht wenige soziale Sektoren in den Städten dazu, das Thema nur unvollständig und teilweise als eine weitere Dimension zu verstehen, ohne die vielfältigen Implikationen zu beachten, die der Neo-Extraktivismus mit sich bringt. Kurz gesagt, es handelt sich um andere Sprachen der Inwertsetzung, die durch ihren Einstieg in die politische und parlamentarische Agenda für einen Riss im Rohstoffkonsens gesorgt haben und eine soziale Resonanz haben, ohne dominierend zu sein. Dennoch wurden die wirtschaftlichen und politischen Erwartungen der breiten Masse, die den Neo-Extraktivismus nähren und die dominanten Modelle der Inklusion durch Konsum möglich machen, auch Teil der öffentlichen Politik.

\section{Neo-Extraktivismus und indigene Gruppen}

Der seit 2000 in der Region registrierte Epochenwechsel, ausgehend von der Denaturierung der Beziehung zwischen Globalisierung und Neoliberalismus, gestaltete ein Übergangs- und Konfliktszenario, in dem einer der wichtigsten Punkte der Rohstoffkonsens war, der sich in der (Re-)Artikulation zwischen Neo-Extraktivismus und einer neuen Version des Entwicklungsdenkens ausdrückte. So wurde die Krise des neoliberalen Konsenses, die erneute Legitimität kritischer Diskurse, 
das Entstehen und die Stärkung verschiedener sozialer Bewegungen, kurz gesagt, die Reaktivierung der populistischen Tradition, in eine neue Phase der Kapitalakkumulation eingefügt, deren Kern der Vormarsch verschiedener Formen des Extraktivismus im großen Stil war.

Dieser Prozess hatte wichtige Konsequenzen in Bezug auf die Situation der indigenen Völker, da das Gegenstück zur Ausweitung der Grenze der kollektiven Rechte, die in den verschiedenen nationalen Verfassungen und internationalen Regelungen (von der ILO-Konvention 169 bis zur Allgemeinen Erklärung der Indigenen Rechte) anerkannt wird, die Ausweitung der Kapitalgrenzen auf indigene Territorien und die Zunahme von sozio-territorialen Konflikten war. Im Rahmen der progressiven Regierungen führte dieses Problem, das zunächst als Spannung und später als Antagonismus gelesen wurde, zu unterschiedlichen Antworten, die im Fall der indigenen Position die Frage nach Autonomie und, allgemeiner, die Frage nach dem Recht auffreie und informierende Konsultation (derecho de consulta previa, libre e informada, CPLI ab hier) angesichts der Ausweitung der Erdöl-, Bergbau-, der Energie- und der Agrarökonomiegrenzen in den Mittelpunkt des Konflikts stellten.

In einem Bericht der ECLAC über die Situation der indigenen Völker, der sich auf die Berichte des UN-Sonderberichterstatters über die Lage der Menschenrechte und Grundfreiheiten der indigenen Völker (2009-2013) stützt, wird als einer der wichtigsten Konfliktpunkte, der sich aus der Expansion der extraktiven Aktivitäten in den indigenen Gebieten ergibt, hervorgehoben, dass »der Staat es versäumt hat, die indigenen Völker zu konsultieren und Maßnahmen zum Schutz ihrer Rechte zu ergreifen, bevor er Konzessionen erteilt oder die Durchführung von extraktiven Projekten autorisiert «. ${ }^{2}$ Dieser Bericht gibt auch

2 Der Bericht stammt aus dem Jahr 2013 und die Kartierung wurde vom Unterstützungsprojekt für den Sonderberichterstatter für die Rechte der indigenen Völker an der Universität von Arizona durchgeführt. Die anderen »Problemknotenpunkte« beziehen sich auf die unzureichende oder nicht vorhandene rechtliche Absicherung der Rechte der indigenen Völker auf ihr Land, ihre Gewässer, ihre natürlichen Ressourcen, ihre biologische Vielfalt und ihr Territorium; auf Konflikte um die Anerkennung heiliger Stätten; auf die nicht vorhandene 
eine Kartierung der extraktiven Industrien wieder, die zeigt, dass in allen Ländern Lateinamerikas, in denen indigene Gebiete existieren, sozio-ökologische Konflikte stattfinden. Im Zeitraum 2010-2013 wurden auf dieser Karte mindestens 226 sozio-ökologische Konflikte in indigenen Gebieten Lateinamerikas identifiziert, die im Zusammenhang mit extraktiven Bergbau-, Erdöl- und Erdgasprojekten stehen (ECLAC 2013, 139).

Die Frage nach der Umsetzung der Konsultation ist jedoch bei weitem nicht eindeutig, da es eine Frage der Auslegung ist, inwiefern diese bindend ist: Sollte dies unverbindlich sein, oder haben die indigenen Völker ein Vetorecht? Die ILO legt fest, dass die Konsultation in gutem Glauben erfolgen muss und dass ihr Zweck darin bestehen muss, die Zustimmung der indigenen Gemeinschaften zu erhalten oder zumindest eine Einigung zu erzielen. Anschließend ging die Erklärung der Vereinten Nationen über die Rechte der indigenen Völker, die 2007 von der UNO verabschiedet wurde, einen Schritt weiter, indem sie den Grundsatz der freien, vorherigen und informierten Zustimmung für die Umsiedlung indigener Gruppen von ihrem Land sowie für die Annahme und Umsetzung von sie betreffenden legislativen und administrativen Maßnahmen unter anderem vorsieht. Darüber hinaus wird den Staaten aufgetragen, Entschädigung für jegliches geistige, kulturelle oder spirituelle Eigentum zu leisten, das indigene Gruppen ohne ihre freie, vorherige und informierte Zustimmung verloren haben. Obwohl diese Bestimmungen nicht verbindlich sind, schaffen sie eine starke Verpflichtung der Staaten und üben Druck auf sie aus, entsprechende Anpassungen vorzunehmen.

In dieser Linie unterscheidet der kolumbianische Soziologe und Jurist César Rodríguez Garavito zwischen einer starken und einer schwa-

oder unzureichende unabhängige Bewertung der ökologischen, wirtschaftlichen und territorialen Auswirkungen von extraktiven Projekten; auf den Ausschluss der indigenen Völker bei der Verteilung der Gewinne aus der Ausbeutung der Ressourcen in ihren Territorien; und schließlich auf die Kriminalisierung des sozialen indigenen Protests gegen Projekte, die ihre Rechte und Cebiete betreffen (ECLAC 2013, 58). 
chen Interpretation des Rechts auf Konsultation. Aus seiner Sicht haben Internationale Gremien wie das UN-Berichtswesen über die Rechte der indigenen Völker und der Interamerikanische Gerichtshof für Menschenrechte (2007) anspruchsvollere Auslegungen des internationalen Rechts festgelegt, insbesondere wenn es um große Entwicklungs- oder Investitionsvorhaben geht, die tiefgreifende Auswirkungen auf ein indigenes Volk haben. Am anderen Extrem haben wir die schwache Verfahrenskonzeption, wie sie das Verfassungsgericht von Ecuador zum Ausdruck bringt (Rodríguez Garavito 2012, 48).

Es existieren auch andere rechtliche Instrumente auf regionaler Ebene, wie zum Beispiel die Inter-Amerikanische Menschenrechtskommission (IACHR) der Organisation Amerikanischer Staaten (OAS) mit Sitz in Costa Rica, die für die amerikanischen Staaten verbindlich ist. Die Berichte des UN-Sonderberichterstatters für die Menschenrechte und Grundfreiheiten der indigenen Völker sind ebenfalls sehr wichtig, da sie den indigenen Forderungen oft Sichtbarkeit und politische Kraft verleihen, indem sie Möglichkeiten zur Überwindung bestehender Hindernisse für den Schutz der Rechte indigener Völker untersuchen, Informationen über Verletzungen dieser Rechte sammeln, Missionen in Konfliktregionen durchführen und Berichte erstellen. Die IACHR hat eine Tradition, dies $\mathrm{zu}$ garantieren. So hat sie beispielsweise 2007 durch die Analyse von fünf strittigen Fällen einen internationalen rechtlichen Rahmen für die Lösung von Problemen zwischen Staaten und indigenen Gemeinschaften ermittelt. Zuerst wurde festgelegt, dass es die Aufgabe der Staaten ist, die effektive Beteiligung der indigenen Völker zu gewährleisten, wozu die Staaten die Pflicht haben, diese Gemeinschaften im Einklang mit ihrer Lebensweise und ihren Traditionen $\mathrm{zu}$ konsultieren, Informationen zu akzeptieren und bereitzustellen und die Kommunikation zwischen den beiden Parteien zu fördern; die Konsultationen sollen in gutem Glauben durch kulturell angepasste Verfahren bereits in den frühen Phasen von Entwicklungsprojekten durchgeführt werden und sicherstellen, dass die Gemeinschaften über die potenziellen Risiken informiert werden. An zweiter Stelle gab sie Empfehlungen zu den zu konsultierenden Themen, unter denen die Ausbeutung der natürlichen Ressourcen in den Gebieten der indigenen 
Völker hervorzuheben ist. Außerdem sollten die Gemeinden selbst und nicht der Staat festlegen, wer die indigene Bevölkerung in jedem Konsultationsverfahren vertritt. Schließlich können die Staaten, wenn der Entwicklungsplan groß angelegt oder von großer Tragweite ist, nicht ohne eine freie, vorherige und informierte Zustimmung vorgehen.

Ein wichtiger Fortschritt auf dem Weg zu einer stärkeren Auslegung war die Entscheidung des IACHR vom 30. Juli 2012 in Bezug auf das Volk der Kichwa Sarayaku im ecuadorianischen Amazonasgebiet. Seit über einem Jahrzehnt gibt es eine Klage gegen den ecuadorianischen Staat, weil dieser eine Ölkonzession erteilt hat und einer argentinischen Gesellschaft erlaubt hat, ohne vorherige Absprache mit dem Volk der Sarayaku seismische Untersuchungen im Territorium der Sarayaku durchzuführen. Das Gericht urteilte, dass Ecuador die Rechte auf vorherige und informierte Anhörung, das Recht auf indigenes Gemeineigentum und die kulturelle Identität verletzt hatte. Der Staat wurde ebenfalls für die erhebliche Gefährdung des Rechts auf Leben und persönliche Integrität sowie für die Verletzung der Rechte auf gerichtliche Garantien und Rechtsschutz zum Nachteil der Sarayaku verantwortlich gemacht. Folglich ordnete das Gericht an, dass Ecuador »den Sprengstoff aus dem Gebiet des Volkes der Sarayaku zu entfernen« habe. Darüber hinaus »muss der Staat angemessene, wirksame und umfassende Anhörungen durchführen, bevor er Projekte zur Rohstoffgewinnung in Angriff nimmt«. Der Staat muss nach wie vor »Pflichtkurse« bezüglich der Rechte der indigenen Völker durchführen, die sich an Beamte richten, die mit indigenen Völkern zu tun haben, und muss »einen öffentlichen Akt der Anerkennung der Verantwortung « wegen den Rechtsverstößen veranstalten. Letztendlich stellte das Gericht fest, dass der Staat 90.000 US-Dollar an materiellem und 1.250.000 US-Dollar an immateriellem Schadenersatz an das Volk der Sarayaku zahlen müsse. ${ }^{3}$

Dieses Urteil stellte einen Meilenstein in diesem Zusammenhang dar und dürfte sich auf bevorstehende Gerichtsverfahren zwischen den indigenen Rechten und dem Ausbau der Rohstoffgrenze auswirken. Es ist also kein Zufall, dass der IACHR seit 2012-2013 unter der Lupe 
lateinamerikanischer Länder steht: So beschloss beispielsweise die venezolanische Regierung unter dem Vorwurf der Voreingenommenheit und moralischen Dekadenz des IACHR ihren Austritt. Gleichzeitig drohte Brasilien mit dem Austritt, nachdem es vom IACHR Schutzmaßnahmen auferlegt bekommen hatte, die die Aussetzung des Baus des Mega-Staudamms Belo Monte beinhalteten, der ohne angemessene Rücksprache mit der indigenen Bevölkerung durchgeführt wurde. ${ }^{4}$

Die Konsultation indigener Völker wird meist in einem immer komplexer und dynamischer werdenden gesellschaftlichen und rechtlichen Konfliktfeld eingesetzt. Aus der Sicht der lateinamerikanischen Regierungen stellt die Konsultation in Zeiten des entwicklungspolitischen Neo-Extraktivismus mehr als nur einen Stein im Schuh dar. Abgesehen von den großspurigen Erklärungen im Namen der indigenen Rechte und der Verteidigung der Pachamama gibt es keine lateinamerikanische Regierung, die nicht versucht hat, die Konsultation einzuschränken, um sie durch verschiedene Gesetze und Verordnungen, die darauf abzielen, einen nicht bindenden Konsultationscharakter zu etablieren, auf seine schwachen Versionen zu beschränken sowie die Bevormundung oder Manipulation in Kontexten starker Machtasymmetrie $\mathrm{zu}$ erleichtern. Dies gilt auch für eine demokratisierende Regierung wie die von Evo Morales, die sich nicht davor scheute, die Konsultation während des Tipnis-Konflikts eindeutig manipulativ zu nutzen. Dies trifft auch für die indigenenfeindliche Regierung von Rafael Correa in Ecuador zu. Ecuador ratifizierte zwar die ILO-Konvention 169, erfüllt die Konsultation in der Praxis aber nicht, sondern setzt vielmehr

4 Es sei daran erinnert, dass der IACHR ein Organ der OAS ist und dass sein Auftrag in der allgemeinen Überwachung verschiedener Themen im Zusammenhang mit den Menschenrechten besteht. In den letzten Jahren wurde der IACHR wegen der Politisierung seiner Urteile stark kritisiert. Er hatte zahlreiche Konflikte mit Venezuela (Klagen wegen Verletzungen der Menschenrechte), Ecuador (Klagen gegen die Pressefreiheit) und Nicaragua (Verletzung der Menschenrechte und mangelnde Rechte der in Costa Rica arbeitenden Nicaraguaner). Ihm wird auch vorgeworfen, nie einen lateinamerikanischen Diktator oder eine lateinamerikanische Diktatur verurteilt zu haben, beispielsweise den Putsch gegen Zelaya in Honduras. 
auf andere Gesichtspunkte, wie etwa die prä-legislative Konsultation. In Peru versuchten aufeinanderfolgende neoliberale Regierungen, von Alan García bis Ollanta Humala, die Forderung nach dem Recht auf Anhörung (gewaltsam) zu bremsen, indem sie dazu tendierten, sie auf die Amazonasvölker zu beschränken. Dies fand zum Nachteil der andinen indigenen Gemeinden statt, von denen viele die Durchführung von Mega-Minenprojekten ablehnen. Dies trifft auch auf Argentinien $\mathrm{zu}$, wo strategische Gesetze zur Regulierung des Rohstoffabbaus (wie das »Kohlenwasserstoff «-Gesetz von 2014, das das Fracking zur Gasförderung ermöglicht) ohne Berücksichtigung von Absprachen mit den indigenen Völkern verabschiedet wurden.

In Bolivien hat die Regierung im März 2015 die Vorschriften für die Partizipation und Anhörung bei Projekten zur Öl- und Gasförderung geändert und damit alle bisherigen Regelungen durch neue ersetzt. Ein weiteres Dekret eröffnete ab Mai desselben Jahres die Öl- und Gasexploration in Schutzgebieten. Außerdem gab es laut CEDIB in sieben Jahren mindestens 49 vorherige Anhörungen ohne Umweltverträglichkeitsprüfung, so dass die betroffenen Bevölkerungsgruppen die Auswirkungen solcher Aktivitäten nicht kennen. ${ }^{5}$ Die so genannte Patriotische Agenda 2025 legt verschiedene Territorien und sogar Schutzge-

5 Pablo Villegas, ein Forscher des bolivianischen Dokumentations- und Informationszentrums (Cedib), berichtete, dass von 2007 bis 2014 etwa 49 Vorberatungen zu Projekten der Erdöl- und Erdgasförderung im Land durchgeführt wurden, ohne die Umweltverträglichkeitsprüfung zu berücksichtigen. Infolgedessen gibt es weiterhin Konflikte in 18 indigenen Gebieten und 11 Schutzgebieten. »Von 2007 bis 2014 wurden nach Angaben des zuständigen Ministeriums 49 Vorabberatungen wegen Projekten zur Öl- und Gasförderung durchgeführt, aber die Umweltverträglichkeitsprüfung ist nicht an die Vorabberatung gebunden«, kritisierte Villegas. »[...] Wie der ANF mitgeteilt hat, erfolgt die Handhabung der Umweltauswirkungen ohne wissenschaftliche Grundlage und wenn es um die Kompensationen geht, dann ohne Umweltverträglichkeitsprüfung, was bedeutet, dass die indigenen Völker nicht wissen, wie sich die Erforschung ihres Territoriums auf sie auswirken wird.«Er fügte hinzu, dass Umweltverträglichkeitsprüfungen eine Art »Staatsgeheimnis« seien und dass der Zugang zu ihnen für Staatsbürger und indigene Völker beschränkt sei. Vollständige Informationen sind erhältlich unter: www.ce- 
biete fest, in denen die indigenen Gemeinden angesiedelt sind. Sogar das Recht auf vorherige Anhörung wurde seines eigentlichen Sinnes beraubt, um die Durchführung von Erdölinvestitionen zu beschleunigen (Gandarillas 2014, 123).

Die Frage der Konsultation ist somit eines der schwierigsten und umstrittensten Themen im internationalen, regionalen und nationalen Regime der Rechte indigener Völker. Obwohl es als »spezialisiertes Instrument « erscheint, ist das internationale System der Rechte indigener Völker in nur zwei Jahrzehnten zum Gegenstand juristischer Auseinandersetzungen geworden, bei denen große wirtschaftliche Interessen sowie das Überleben indigener Völker und anderer ethnischer Gruppen auf dem Spiel stehen.

Die Situation der indigenen Völker ist in ein Szenario voller Gegensätze und Widersprüche eingefügt. Die Anerkennung kollektiver Rechte eröffnet in Lateinamerika zahlreiche Debatten über die Prozesse der pluralistischen Demokratisierung der lateinamerikanischen Gesellschaften im 21. Jahrhundert und insbesondere über die Tragfähigkeit und Reichweite dieser international proklamierten Rechte, die in allen heute geltenden politischen Verfassungen Lateinamerikas verankert sind. Wir stehen also vor einem Dilemma, das die Kollision zweier Dynamiken widerspiegelt. Auf der einen Seite haben wir auf globaler Ebene seit Mitte des 20. Jahrhunderts und im Zuge des Dekolonisierungsprozesses die Erweiterung der Grenzen der kulturellen und politischen Rechte der indigenen Völker erlebt. Horizontale Konzepte wie etwa Autonomie, kollektive Rechte und der plurinationale Staat durchziehen die indigene Narrative und verdeutlichen das wachsende politische Empowerment der indigenen Völker in unterschiedlichen lateinamerikanischen Ländern. Andererseits gibt die schwindelerregende Ausdehnung der Kapitalgrenzen in einem extraktivistischen Sinne Rechenschaft über einen neuen Prozess der Verdrängung der indigenen Völker, der in seiner Gesamtheit die Erhaltung der grundlegenden Ressourcen des Lebens bedroht. Enteignung, Re-Kolonisierung,

dib.org/post_type_titulares/cedib-en-7-anos-hubo-al-menos-49-consultasprevias-sobre-hidrocarburos-sin-evaluacion-ambiental-pagina-siete-17-8-15/. 
das sind einige der Begriffe, die immer wieder entweder von zahlreichen indigenen Aktivisten oder von sozialen Bewegungen verwendet werden. Zusammenfassend kann man sagen, dass die Ausdehnung der Rohstoffgrenze die Möglichkeit der Anwendung der kollektiven Rechte der indigenen Völker, die auf globaler, nationaler und lokaler Ebene schon kaum anerkannt werden, zunichtemacht.

\section{Populare Feminismen des Südens}

Historisch und auch heute ist die Rolle der Frauen in den sozialen Bewegungen des globalen Südens zentral. In Lateinamerika hat der Protagonismus der Frauen in den letzten Jahrzehnten zugenommen: Indigene Frauen, Bäuerinnen, Afro-Frauen, von Armut betroffene Frauen auf dem Land und in der Stadt, Transgender und lesbische Frauen haben ihr Schweigen beendet, sich mobilisiert und stellen Solidaritätsbeziehungen und neue Formen der kollektiven Selbstverwaltung her. Um dieses Empowerment hervorzuheben, wird immer häufiger von popularen Feminismen gesprochen, die über ihre Unterschiede hinaus mit den am meisten marginalisierten Sektoren verbunden $\mathrm{zu}$ sein scheinen und dazu neigen, die individualistische und modern-westliche Vision zugunsten einer größeren Wertschätzung der »kollektiven und gemeinschaftlichen Erfahrung« in Frage zu stellen (Korol 2016).

$\mathrm{Zu}$ den möglichen Formen, die die popularen Feminismen in der Region annehmen, gehören Gemeinde-Feminismen. Diese betonen die Existenz anderer Formen der Moderne und unterscheiden sich von der dominanten westlichen Variante, indem sie die Entkolonialisierung mit der Entpatriarchalisierung verbinden. Den Kern bilden dabei feministische Gruppen, die das Patriarchat mit der Kolonialgeschichte verbinden; andere, im Gegenteil, weit entfernt von jeder Idealisierung der Gemeinschaft, heben ihre »Refunktionalisierung« (Lorena Cabnal, guatemaltekische Xinca-Feministin) oder ihre »koloniale Verbindung« 
(Julieta Paredes, Feministische Versammlung, Bolivien) im Rahmen der indigenen Bauerngemeinschaften hervor. ${ }^{6}$

Im Einklang mit diesem Empowerment erlangten lateinamerikanische Frauen im Zuge der Ausbreitung sozio-ökologischer Konflikte immer mehr Aufmerksamkeit und federführende Bedeutung. Als Beispiel kann der Fall Argentiniens angeführt werden, wo die Bewegung der Mütter des Barrio Ituzaingó aus der Stadt Córdoba eine Vorreiterrolle bei der Anprangerung der Auswirkungen von Glyphosat auf die Gesundheit spielte, was zu dem ersten Strafprozess zu diesem Thema führte (Svampa und Viale 2014). Erwähnenswert sind auch die Hartnäckigkeit der Frauen der Versammlung von Chilecito und Famatina (Lehrerinnen, Hausfrauen, Ladenbesitzerinnen), die sich gegen die Attacken von Bergbaukonzernen wehrten (Sie vertrieben zwischen 2009 und 2015 vier Unternehmen, darunter Barrick Gold); schließlich auch der Widerstand der Mapuche-Frauen gegen das Fracking in Neuquén (Cristina Lincopan, inzwischen verstorben, und Relmu Nancu, die 2015 wegen versuchten Mordes vor Gericht gestellt wurde). In Chile ist dies der Fall bei den Mujeres de Zonas de Sacrificio en Resistencia de QuinteroPunchacaví (dt. Frauen aus geopferten Gebieten in Quintero-Punchacaví im Widerstand). Es handelt sich um eine Bewegung im Umkreis einer Industriezone in der Nähe von Valparaíso, die von von Paola Bolados und Alejandra Sánchez Cuevas (2017) im Hinblick auf feministische politische Ökologie und Umweltgewalt analysiert wurde. Dasselbe kann man von Kolumbien bezüglich des Frauenwiderstandes gegen die Ausdehnung der Erdölgrenze (Roa, Roa, Toloza und Navas 2017) sagen.

Dies sind nur einige Beispiele, die unterstreichen, dass sich der weibliche Protagonismus in den ökoterritorialen Konflikten in allen Ländern der Region wiederfindet. Es sind persönliche und zugleich kollektive Stimmen, und ein aufmerksames Zuhören eröffnet nach

6 Im Feld des Cemeindefeminismus, wie von Gargallo (2015) hervorgehoben, hat sich der bolivianische Feminismus von La Paz und Cochabamba durch seine kontinentale Wirkung ausgezeichnet: die Cruppe der Mujeres Creando und später auch die Feministische Versammlung (Asemblea Feminista) sind hier hervorzuheben. 
und nach verschiedene Ebenen des Denkens und Handelns. Denn hinter der Anklage und der Bezeugung konkreter Missstände steht nicht nur der einvernehmliche Kampf der Frauen in den Territorien, der eine starke Identifikation mit dem Land und seinen vitalen Reproduktionszyklen mit sich bringt. Vielmehr zeigt sich hier auch auch die Dekonstruktion des Mythos von Entwicklung und die Konstitution einer anderen Beziehung zur Natur. In einem Hin und Her vom Öffentlichen zum Privaten besteht die Forderung nach einer freien, ehrlichen Stimme, »einer eigenen Stimme« (Gilligan 2015), die das Patriarchat in all seinen Dimensionen hinterfragt und versucht, die Ethik der Versorgung an einen zentralen und befreienden Ort $\mathrm{zu}$ stellen, der auf unbestreitbare Weise mit der menschlichen Existenz verbunden ist. Sicherlich bringt der prozessuale Charakter der Konflikte eine Infragestellung des Patriarchats mit sich, sozusagen als Modell der Herrschaft eines Geschlechts über ein anderes, über eine binäre und hierarchische Matrix, die das Männliche vom Weiblichen trennt und privilegiert. $^{7}$

Mit anderen Worten, die Kämpfe der Frauen (in indigenen und bäuerlichen Organisationen, sozio-ökologischen Bewegungen, UmweltNGOs und kulturellen Kollektiven) schaffen eine andere Beziehung zwischen Gesellschaft und Natur, in der der Mensch nicht als eine Einheit außerhalb der Natur, sondern als ein Teil von ihr verstanden wird. Der Übergang zu einer beziehungsorientierten Sichtweise stellt die Idee der Interdependenz in den Mittelpunkt, die bereits als ökologische Dependenz redefiniert ist und ein Verständnis der menschlichen Realität durch Anerkennung und Sorgfalt mit Anderen und mit der Natur vermittelt.

Schließlich verweisen mehrere Autorinnen auf die wachsende Bedeutung der südlichen Feminismen, darunter Vandana Shiva und Maria Mies (1998), die oft vom Ökofeminismus des Überlebens sprechen, ver-

Es ist nicht einfach für diese kämpfenden Frauen, sich als Feministinnen zu erkennen. Zunächst nehmen sich die Frauen nicht explizit als Feministinnen wahr, erst in einem zweiten Moment der Kämpfe, im Hin- und Herschwanken vom Öffentlichen ins Private, treten sie auch offen als Feministinnen hervor. 
bunden mit den vielfältigen Erfahrungen der Frauen bei der Verteidigung von Gesundheit, Überleben und Territorium. Das hat zu dem Bewusstsein geführt, dass es starke Verbindungen zwischen Frauen und Umweltschutz sowie Feminismus und Ökologie gibt. In dieser Linie ist es interessant, die Verbindungen zwischen den popularen Feminismen des Südens und der ökofeministischen Perspektive zu untersuchen. Obwohl der Begriff der Ökofeministin in den 1970er Jahren entstand und es viele Autorinnen gibt, die sich in diesen Bereich einordnen, sind ihre Beiträge erst in den letzten Jahren stärker verbreitet worden. Der Ökofeminismus greift die Diagnose der ökologischen Krise wieder auf, die er als eine soziale Krise anthropologischen Charakters versteht, die aus der doppelten Dominanz des Menschen auf der Ebene der zwischenmenschlichen Beziehungen sowie im Bereich der Beziehung des Menschen zur Natur entstanden ist. Auf dieser Grundlage liefert der Ökofeminismus eine ähnliche Interpretation des Verhältnisses zwischen der Dominanz eines Geschlechts über ein anderes und der des Menschen über die Natur, die sich in einer identitären Logik ausdrückt, die die Abwertung und Marginalisierung der als anders angesehenen Personen rechtfertigt: Frauen gegenüber Männern und das Natürliche gegenüber dem Menschen.

Schließlich ist zu klären, dass es innerhalb des Ökofeminismus verschiedene Strömungen gibt, die vom differenzialistischen oder identitären Feminismus, der die Beziehung zwischen Frau und Natur naturalisiert, bis zum konstruktivistischen Ökofeminismus ${ }^{8}$ reichen, der sie als historisch-soziale Konstruktion begreift, die mit der geschlechtlichen Arbeitsteilung verbunden ist. Aus meiner Sicht ist es wichtig, nicht in eine essentialistische Vision der Beziehung zwischen Frau und Natur zu geraten. Denn der Schlüssel ist nach wie vor das Feld der

8 Siehe in dieser Linie Aguinaga, Lang, Mokrani und Santillana (2012), Daza, Ruiz und Ruiz (2013), die die Erfahrungen der populären Feminismen in Peru sammeln und sie mit den Ökofeminismen verbinden. Im Falle Argentiniens wurde die Rolle von Frauen in sozio-ökologischen Bewegungen aus der Perspektive des Ökofeminismus von Bilder (2013) aufgezeigt. 
Wahlverwandtschaften, die durch die Ausnutzung der ungleichen Arbeitsteilung und die Trennung zwischen Produktion und Reproduktion des Sozialen entstehen. Es muss jedoch gesagt werden, dass es in Lateinamerika eine wichtige Präsenz von popularen und kommunitären Feminismen spiritueller Ausrichtung gibt, die bestimmte Elemente der essentialistischen Perspektive wieder aufgreifen, »aber ohne das Männliche zu verteufeln «(Puleo 2011), und die vor allem die Identifikation mit dem Territorium und die Verteidigung der Lebenszyklen hervorheben. 



\section{Kapitel 4: Auf dem Weg zu einem Neo-Extraktivismus der Extremfiguren}

In diesem Kapitel schlage ich vor, einige der Ausdrucksformen der gegenwärtigen Verschärfung des Neo-Extraktivismus durch seine extremen Zahlen zu lesen. Hierzu zählt unter anderem die Zunahme der staatlichen und parastaatlichen Repression, die sich in der Ermordung von UmweltaktivistInnen, der Entstehung neuer krimineller Territorialitäten, die mit dem illegalen Bergbau oder informellen Kleinbergbau und der Stärkung der patriarchalischen Struktur in einem Kontext der Vermännlichung der Territorien verbunden sind. In Ergänzung zu dieser Lesart in einem geopolitischen Zusammenhang wird im letzten Abschnitt die Erweiterung der Fördergeografie in den zentralen Ländern dargestellt.

\section{Der Vormarsch extraktivistischer Gewalt}

Um 2008-2010 erleben wir eine Phase der Vervielfältigung von extraktivistischen Projekten, was sich in den verschiedenen nationalen Entwicklungsplänen widerspiegelt. Sie sind Teil des Wahlprogramms der verschiedenen lateinamerikanischen SpitzenpolitikerInnen, von denen nicht wenige eine Wiederwahl anstreben: von Juan Manuel Santos' »Energie-Minen-Lokomotive« (Nationaler Entwicklungsplan, 2010-2014) in Kolumbien, über Cristina Fernández de Kirchners Strategischer Agrar-Lebensmittel-Plan 2020 (pea2) in Argentinien, bis zum Orinoco-Minenbogen in Venezuela - der erstmals im Entwick- 
lungsplan von Hugo Chávez formuliert wurde und dann von Nicolás Maduro wieder aufgegriffen wurde - und dem Übergang vom "großen Industriesprung« (2010) zur Patriotischen Agenda Boliviens für 2025 (2015). Tatsache ist, dass die lateinamerikanischen Länder auf die exponentielle Zunahme von extraktiven Megaprojekten setzen, die die wahllose Ausbeutung von Naturgütern mit Exportzielen fördert.

Die Kehrseite dieses Prozesses war die Zunahme von Konflikten, die direkt oder indirekt zur Kriminalisierung von sozio-ökologischen Konflikten und zur Zunahme von staatlicher und parastaatlicher Gewalt beitrugen. Laut Global Witness (2014) gab es zwischen 2002 und 2013 weltweit 908 dokumentierte Morde an UmweltaktivistInnen, von denen 83,7 \% (760 Fälle) in Lateinamerika stattgefunden haben. Die Daten zeigen auch, dass der Anstieg ab 2007 und noch mehr im Jahr 2009 stattfand, das heißt in Übereinstimmung mit der Multiplikationsphase der extraktiven Projekte, wie sie sich in den verschiedenen Entwicklungsprogrammen der einzelnen Regierungen widerspiegeln.

Nach Brasilien (50 Todesfälle) und den Philippinen (33) liegt Kolumbien mit 26 Morden an UmweltschützerInnen im Jahr 2015 an dritter Stelle des Rankings. Die Rangliste umfasst auch Länder wie Honduras, Nicaragua, Panama, Mexiko, Guatemala und Peru. So kam es Anfang 2012 in Panama zu starken Repressionen, die zwei Mitgliedern der indigenen Gemeinschaft der Ngäbe-Buglé das Leben kosteten. In Peru wurden während der Regierung von Ollanta Humala (2011-2016) 25 Menschen in einem Klima der Repression getötet. Besonders betroffen war die Region Cajamarca, wo sich die Bevölkerung gegen das CongaProjekt organisierte. Im März 2016 wurde Berta Cáceres, Gründerin der Organisation COPINH, von den Schergen der Repression in Honduras ermordet, weil sie sich dem Bau eines Wasserkraftwerks widersetzte. ${ }^{1}$

1 Im Jahr 2015 erhielt Berta Cáceres als Anerkennung für ihren Kampf den Goldman-Preis, auch als grüner Nobelpreis bekannt. Cáceres gründete die COPINH. Dieser Organisation und dem Volk der Lenca ist es gelungen, das weltgrößte Wasserkraftunternehmen, die chinesische Firma Synohidro, dazu zu bewegen, sich aus dem Bauprojekt des Agua Zarca-Staudamms am Fluss Gualcarque zurückzuziehen. 
Im Januar 2017 wurde Laura Vásquez Pineda, eine Feministin und Aktivistin gegen die Mega-Bergbauindustrie und Mitglied des Netzwerkes der Ahnenheilerinnen des Gemeindefeminismus in Guatemala, ermordet. Ebenso wurde in Argentinien unter der konservativen Regierung von Mauricio Macri im Jahr 2017 in einem Kontext sich verhärtender Landstreitigkeiten der Mapuche Rafael Nahuel von den staatlichen Streitkräften ermordet, und ein weiterer junger Mann, Santiago Maldonado, ertrank in einer Situation staatlicher Repression. ${ }^{2}$

Der Neo-Extraktivismus fordert immer mehr Opfer in der globalisierten Peripherie, besonders in Lateinamerika, das hier eine weltweite Spitzenstellung einnimmt. Wie in anderen Zeiten verwandelt sich die eldoradistische Illusion in eine erneute Dialektik von Enteignung und Abhängigkeit, die mit mehr extraktiven Aktivitäten, mehr Gewalt und weniger Demokratie einhergeht. Dieser Prozess hat sich verstärkt: Allein 2016 fanden von 200 Morden an UmweltaktivistInnen 60 \% in Lateinamerika statt; diese Dimensionen bestätigten sich für $2017 .{ }^{3}$ Nichts deutet darauf hin, dass sich diese Indizes verbessern werden, ganz im Gegenteil, wenn man die derzeitige konservative Wende in Ländern wie Argentinien und Brasilien berücksichtigt, deren derzeitige Regierungen nicht nur das extraktivistische Modell in all seinen Versionen verstärkt haben, indem sie die staatliche Gewalt gegen die verwundbarsten Bevölkerungsgruppen verschärften, sondern auch eine Reihe von politischen Maßnahmen ergriffen, die einen erheblichen Rückschritt in Bezug auf die sozialen Rechte mit sich bringen.

2 Seit Beginn der Regierung Macri haben sich die Konflikte verschärft und viele Mapuche-Anführer wurden vor Gericht gestellt. Außerdem gab es im Jahr 2017 zwei Todesfälle in einer Repressionssituation.

3 Siehe: https://www.theguardian.com/environment/ng-interactive/2017/jul/13/ the-defenders-tracker und www.jornada.unam.mx/ultimas/2018/02/02/asesinan-acerca-de-200-defensores-del-medio-ambiente-en-2017-globalwitness-5318.html. 


\section{Enklaven und kriminelle Territorialitäten}

Die Dynamik der Enklave, die mit dem Extraktivismus verbunden ist, hat eine lange Geschichte in der Region, die sich vor allem auf die Gewinnung von Mineralien und den Export verschiedener Rohstoffe (Zuckerrohr, Guano, Kautschuk, Holz und andere) bezieht. Dorf-Lager, manchmal in Städte umgewandelt, erfahren über Nacht die Pracht und Verschwendung, die Armut und den extremen Reichtum. Aber wenn schließlich die Lichter ausgehen und sich das Kapital auf der Suche nach billigen Rohstoffen in andere Breitengrade zurückzieht, bieten solche Territorien oft das wiederholte Bild von Plünderung und Enteignung: Postkarten eines stark degradierten Territoriums, verwandelt in eine Opferzone, die den lokalen Gemeinschaften nur noch die desolaten ökologischen und sozial-gesundheitlichen Auswirkungen als Erbe hinterlässt. Es handelt sich um die Kapitalzyklen, die durch die Ausweitung der Rohstoffgrenze gekennzeichnet sind, ein historisches geographisches Modell, das auf einer raschen Aneignung (Moore 2013) beruht und nach Erschöpfung der Ressource eine erneute geographische Ausdehnung und Diversifizierung anstrebt.

$\mathrm{Zu}$ Beginn des 20. Jahrhunderts trat zur Dynamik der Enklave, die traditionell mit der Minen- und Plantagenwirtschaft verbunden war, der Ölkreislauf hinzu, wenngleich auch die verschiedenen Nationalisierungsprozesse, die in Lateinamerika um die 1940er und 1950er Jahre stattfanden, eine neue Etappe eröffneten, die auf einem Verständnis nationaler Souveränität basierten. In der gegenwärtigen Ära scheinen der minerale Neo-Extraktivismus und zum Teil auch die Erdölindustrie den eher klassischen Weg der Export-Enklave $\mathrm{zu}$ beschreiten, verbunden mit einer beschleunigten Akkumulation und Ausdehnung der Fördergrenze. Aus sozialer Sicht wird die Konfiguration der Abbaugebiete durch die Verschiebung des früheren wirtschaftlichen und sozialen Gefüges und die Verfestigung einer starken Struktur von Ungleichheiten deutlich. Dies umfasst verschiedene Aspekte, die mit dem Lebensstil und der Lebensqualität sowie den Arbeits- und Geschlechterverhältnissen zusammenhängen. Die außerordentliche Rentabilität der Export-Enklaven führt zu einem deutlichen Anstieg 
der Lebenshaltungskosten, was die Lohnunterschiede zwischen den hoch bezahlten Öl- und/oder BergarbeiterInnen auf der einen Seite und der übrigen Bevölkerung auf der anderen Seite noch verschärft. Der Öl-/Mineralienboom bringt als Korrelat auch eine Immobilienkrise und hohe Mietpreise mit sich. Letztlich sind die soziale Zerrüttung und die Organisation der Arbeitszeit miteinander verflochten, was soziale Probleme wie Drogen-, Alkohol- und Glücksspielabhängigkeiten hervorruft.

Tatsächlich haben extraktive Territorien oft eine eigene Struktur, die sich von der Struktur der nicht-extraktiven Territorien unterscheidet, da sie soziale Probleme, die bereits in der Gesellschaft existieren, verstärken. $\mathrm{Zu}$ diesen gehören Lohnunterschiede, hohe Preise, Abhängigkeiten, zunehmende Kriminalität und Prostitution. In jüngerer Zeit kommen der Menschenhandel und die Ausbreitung krimineller Netzwerke hinzu.

Im neuen Jahrhundert gab es Veränderungen verschiedener Art auf nationaler und globaler Ebene. Der soziale Umbruch und die soziale Desorganisation, die unsere Gesellschaften erleben, haben notorische Veränderungen im sozialen Gefüge hervorgerufen. Unsere Gesellschaften sind viel stärker fragmentiert, was mit der Explosion des Drogenhandels, mit dem Fortbestehen von Ungleichheiten und der Marginalisierung sowie mit der wachsenden Präsenz des Problems der Unsicherheit noch komplexer geworden ist. In der Folge wurden auch die Formen der kollektiven Gewalt verstärkt und diversifiziert. So findet die Intensivierung dieser Gewaltketten ${ }^{4}$ einen fruchtbaren Boden in ex-

In dem von Javier Auyero und María Fernanda Berti veröffentlichten Buch La violencia en los márgenes wird der Begriff der Cewaltketten eingeführt, »der sich auf die Art und Weise bezieht, wie verschiedene Arten von Gewalt, die gewöhnlich als getrennte und analytisch unterschiedliche Phänomene gedacht werden, miteinander verbunden sind und aufeinander reagieren « (Auyero und Berti 2013, 94). Aus meiner Sicht neigen diese Gewaltketten, die die Autoren als Teil des täglichen Lebens der am stärksten benachteiligten Viertel analysieren, dazu, an Ausmaß zu gewinnen und sich mehr und mehr auf die gesamte Gesellschaft auszudehnen. Dies hängt auch mit der Unfähigkeit der Staaten zusammen, demokratische und zugleich befriedigende Antworten auf die 
traktiven Kontexten, in denen sie sich gewöhnlich in extremen Zahlen ausdrückt, das heißt in der Entstehung neuer krimineller Territorien, in denen Marginalität - und Komplizenschaft - bezüglich des Zentralstaates mit räuberischem Extraktivismus und der Suche nach außerordentlicher Rentabilität verbunden ist.

Dieses Phänomen ist heute in bestimmten marginalisierten Regionen Venezuelas zu beobachten, in denen sich die Rohstoffgrenze ausweitet (Teran 2016). Bedenken wir, dass ab 2013, mit dem Ende des so genannten Rohstoff-Superzyklus, mehrere Regierungen angesichts des Handelsbilanzdefizits eine neue extraktive Wende vollzogen haben. Wie Edgardo Lander hervorhebt, war der wichtigste - wenn auch nicht der einzige - Auslöser für die Krise in diesem Land der drastische Rückgang des internationalen Erdölpreises. ${ }^{5}$ In diesem Sinne begann die Regierung von Nicolás Maduro in Venezuela eine intensive Suche nach Devisen und schuf in Übereinstimmung mit dem Plan de la Patria (2013-2019) im Februar 2016 per Dekret eine neue nationale strategische Entwicklungszone, den "Arco Minero del Orinoco« (Orinoco Minenbogen), womit fast $112.000 \mathrm{~km}^{2}$, also $12 \%$ des nationalen Territoriums, für den Abbau von Gold, Diamanten, Coltan, Eisen und anderen Mineralien in großem Maßstab geöffnet wurden. Um ausländische Investitionen anzuziehen, unterzeichnete die Chavista-Regierung Allianzen und Abkommen mit 150 nationalen und transnationalen Unternehmen, deren Inhalt nicht bekannt ist, da der Ausruf des Ausnahmezustands und des wirtschaftlichen Notstands für den Arco Mine-

durch diese Gewaltformen verursachten Probleme zu geben. Anders ausgedrückt: In einem Kontext der Ungleichheit werden die Gewaltketten verstärkt und die Beziehung zwischen verschiedenen Situationen der Ausgrenzung, Enteignung und Unterwerfung hervorgehoben, was einen beunruhigenden demokratischen Rückschritt und sogar die Gefahr der Faschisierung bestimmter Bereiche der Cesellschaft offenbart. José Manuel Valenzuela (2018) sieht in diesen kriminellen Territorialitäten eine Nekropolitik am Werk, die vor allem Jugendliche trifft.

5 Siehe den Aufruf vom April 2016 zur Beendigung des Ökozids am Orinoco Minenbogen unter https://entitleblog.org/2016/04/18/llamado-a-adhesionesdetener-el-ecocidio-minero-en-la-cuenca-del-orinoco-venezuela/. 
ro Verträge mit Ermessensspielraum zulässt, die keine Genehmigung durch die Nationalversammlung erfordern. So wurde die Ausdehnung der Rohstoffgrenze durch Mega-Bergbau als neue "magische« Lösung bei der Suche nach einer Diversifizierung der jetzt in der Krise befindlichen Erdölförderung präsentiert. Laut Terán würde dies eine neue extraktive Kartierung entwerfen, bei der »die neue Aneignung der Grenze über die historische Karte hinausgeht und sich auf Territorien von Naturschutzgebieten, Offshore-Extraktionen und Nationalparks ausdehnt « (Terán 2016, 261).

Doch über die geplanten Megaprojekte im Orinoco-Minenbogen hinaus ist es eine Tatsache, dass in dieser Region neue Territorialitäten entstanden sind, die neue Extremfiguren des Extraktivismus darstellen. Jüngste Untersuchungen weisen gleichzeitig auf das Entstehen und die Verfestigung von kriminellen Banden hin, die mit dem illegalen Minenbetrieb in Verbindung stehen. Obwohl es nicht das erste war, trug das Massaker von Tumeremo im Bundesstaat Bolivar im Jahr 2016 mit der tragischen Bilanz von 28 getöteten Minenarbeitern (Pardo 2016) dazu bei, die wachsende Beziehung zwischen Rentismus, Kriminalität und manuellem und illegalem Minenbetrieb sichtbar zu machen, ein Phänomen, das sich in den letzten zehn Jahren zugespitzt hatte (Romero und Ruiz 2018).

Das, was heute bereits als pranato minero ${ }^{6}$ bekannt ist, zeigt die Konturen einer neuen extraktiven, gewalttätigen und mafiösen Territorialität, die als Gegenpol einen Staat mit geringer Regulierungs- und territorialer Kontrollfähigkeit hat, der gleichzeitig Beziehungen zu bewaffneten Banden unterhält. Wir stehen also vor der Entstehung einer parastaatlichen Sphäre - von unten -, die eine große Anzahl von legalen und illegalen Wirtschaftsakteuren und sozialen Subjekten einbezieht. Solche kriminellen Strukturen kontrollieren nicht nur die Territorien, sondern auch die Bevölkerung und die Subjektivitäten, was einen schweren Schlag für jeden Versuch der Rekonstruktion eines demokratischen

6 Genau genommen bedeutet pran im Gefängnis-Jargon »Chef«. Das Kurzwort wird oft mit »preso reincidente asesino nato « übersetzt, was auf Deutsch in etwa »geborener Killer und Rückfallhäftling« bedeuten würde. 
Projekts bedeutet. Und all dies geschieht noch bevor die transnationalen Unternehmen mit ihrer räuberischen Logik in die Territorien eindringen.

Die Ausweitung der kriminellen Strukturen im Zusammenhang mit dem illegalen Minenbetrieb ist jedoch keine spezifische Figur für Venezuela, denn sie ist auch in Peru zu finden, wo die kriminellen Organisationen des Landes 2016 profitabler waren als die Netzwerke des Drogenhandels. ${ }^{7}$ Allerdings nimmt das Phänomen in Venezuela eindringlichere Züge an, und zwar als Folge der Staatskrise und aufgrund des phänomenalen wirtschaftlichen Debakels, das verschiedene Bevölkerungsgruppen auf der Suche nach neuen Überlebensstrategien vertreibt. Zusammenfassend kann diese Art der sozio-territorialen Konfiguration als Extremfigur des Extraktivismus begriffen werden, die sich durch soziale Desorganisation, Ungleichheit, maximale Überaneignung und die Stärkung der patriarchalischen Herrschaftsmatrix auszeichnet, die die bereits in der Gesellschaft existierenden Gewaltketten verstärkt.

Nicht weniger gravierend ist dagegen die Kombination aus politischem Klientelismus und extraktiver Gewalt, die das qualvolle Verhältnis zwischen der bolivianischen Regierung und den mächtigen Minenkooperativen im Streit um den Mehrwert, der nach der Zeit der außerordentlichen Rentabilität entstanden ist, kennzeichnet. Die Ermordung des stellvertretenden Regierungsministers Rodolfo Illanes durch einige Genossenschaftsmitglieder im Jahr 2016 als Vergeltung für ein gewaltsames Vorgehen der Polizei war eine Nachricht, die große nationale und internationale Auswirkungen hatte. Zweifellos handelte es sich um einen Rohstoffkrieg, da es vor dem Hintergrund sinkender internationaler Rohstoffpreise um die Kontrolle des Mehrwerts ging. Wie ein Zauberlehrling musste sich die Regierung von Evo

7 "Sie verdienten 2,6 Milliarden US-Dollar aus der Produktion und dem Verkauf von illegal gewonnenem Cold, während die Netzwerke, die sich dem Drogenhandel widmen, Gewinne von 500 Millionen bis 1 Milliarde US-Dollar hatten.« La República, 25. April 2017. Zugang am 8. März 2018. http://larepublica.pe/sociedad/1035115-mineria-ilegal-genero-mas-ganancias-que-el-narcotrafico. 
Morales mit einem exzessiven Korporativismusmodell des unternehmerischen Typs auseinandersetzen, das sie selbst durch wirtschaftliche Privilegien im Austausch gegen politische Unterstützung stärkte. Es sollte klargestellt werden, dass viele dieser Verbände nicht einmal Genossenschaften sind, sondern verdeckte Privatunternehmen, die unter überaus ausbeuterischen Bedingungen, die lange Arbeitszeiten (bis zu 16 Stunden pro Tag) einschließen, Arbeit an Subunternehmer vergeben und das gewonnene Material an transnationale Unternehmen verkaufen. Nach Angaben des Cedib gibt es zwischen 100.000 und 120.000 genossenschaftliche Minenarbeiter, aber ein großer Teil (zwischen $40 \%$ und $50 \%$ ) steht bei Subunternehmen unter Vertrag. So zeigt die Realität das Entstehen eines Eigentumssektors, der durch die Ausbeutungsbedingungen und die hohen Preise der Mineralien während des Superzyklus der Rohstoffe angetrieben wird. Nach Gas ist der Bergbau heute Boliviens zweitgrößte Wohlstandsquelle mit $25 \%$ der Exporte, darunter Zinn, Zink, Silber, Kupfer und Gold. Mit dem Wirtschaftsboom ist die Zahl der Genossenschaften von 500 im Jahr 2005 auf 1.600 im Jahr 2015 gestiegen.

\section{Das andere Gesicht des Patriarchats: Extraktivismus und Gewaltketten}

Es besteht ein historischer Zusammenhang zwischen Erdöl- und Minenaktivität, der Maskulinisierung der Territorien und der Verfestigung des Patriarchats. Tatsächlich neigt die Prostitution im Kontext einer starken Konzentration der männlichen Bevölkerung dazu, naturalisiert zu werden, das heißt, nicht als ein soziales und kulturelles Problem angesehen zu werden. Dazu kommt, dass in den letzten Jahrzehnten, im Kontext der Globalisierung der kriminellen Netzwerke, die Prostitution und der Menschenhandel zugenommen haben. Frauenhandel für die Sexindustrie generiert große Gewinne in einem zunehmend globalisierten Kreislauf der Kriminalität (Sassen 2003b), der die Komplizenschaft und - illegale - Beteiligung verschiedener Machtakteure (politisch und rechtlich) beinhaltet. 
In diesem Sinne besteht in der gesamten Region ein unmittelbarer Zusammenhang zwischen Bergbau, Prostitution und zunehmendem Menschenhandel. In Ländern wie Bolivien, Peru, Kolumbien und Mexiko wird das Menschenhandelsnetz hauptsächlich mit dem illegalen Bergbau in Verbindung gebracht. Dies ist in der Region Puno in Bolivien der Fall, wo Tausende von Fällen von Frauenhandel und sexueller Ausbeutung gemeldet wurden. Wie Livia Wagner, Autorin des Berichts >Organisierte Kriminalität und illegaler Minenbetrieb in Lateinamerikar, feststellt, "gibt es eine starke Verbindung zwischen illegalem Minenbetrieb und sexueller Ausbeutung. Immer wenn es große Migrationen von Männern in ein Gebiet gibt, gibt es eine große Nachfrage nach sexuellen Dienstleistungen, die oft Sexhandel generiert « (Miranda 2016). Dies geschieht auch in den Minengebieten Perus, wie beispielsweise in der Amazonasregion Madre de Dios, wo illegaler Goldabbau betrieben wird. Im Falle Argentiniens folgen Menschenhandel und Prostitution dem gleichen Weg wie die Erdöl- und Minenroute sowie die Sojaroute.

Dem muss die Zunahme staatlicher und parastaatlicher Gewalt gegen Frauen, die sich dem Neo-Extraktivismus widersetzen, hinzugefügt werden. Es wurde bereits festgestellt, dass die Kriminalisierung, Übergriffe und Ermordung von bzw. auf UmweltschützerInnen in der Region deutlich zugenommen hat. Zwischen 2011 und 2016 registrierten Menschenrechtsorganisationen 1.700 Angriffe auf Umweltschützerinnen in Süd- und Mittelamerika (Maldonado 2016). Die meisten dieser Aggressionen wurden im Zusammenhang mit Zwangsräumungen verübt, bei denen Frauen von Polizeikräften oder paramilitärischen Gruppen körperlich und sexuell misshandelt wurden (Urgent Action FundLatin America 2017).

Eines der schockierendsten Verbrechen der letzten Zeit war das an Berta Cáceres der COPINH in Honduras. Ein weiterer eklatanter Fall von Verfolgung und Einschüchterung ist der von Máxima Acuña in Peru, einem Mitglied der Vereinigung der Frauen zur Verteidigung des Lebens und der Lateinamerikanischen Frauenunion (ULAM), die sich ge- 
gen das Conga-Megamining Projekt wehrt. ${ }^{8}$ Schließlich ist der Fall der AktivistInnen von Acción Ecológica hinzuzufügen, einer international anerkannten ecuadorianischen NGO, die sich der Forschung und Beratung verschrieben hat und fast ausschließlich aus Frauen besteht. Sie hat wegen ihres Kampfes für die Rechte der Natur und für die Gemeinden, die gegen den Neo-Extraktivismus kämpfen, zwei Auflösungsversuche der Regierung von Rafael Correa (2009 und 2016) erlebt.

Zusammenfassend lässt sich sagen, dass dort, wo die durch die Maskulinisierung der Territorien und außerordentliche Profite gekennzeichneten extraktiven Aktivitäten eintreten, die verschiedenen sozialen Probleme, die bereits in der Gesellschaft vorhanden sind, intensiviert und verschärft werden. So ist eine der Folgen die $\mathrm{Zu}$ spitzung der Stereotype der geschlechtlichen Arbeitsteilung, die die Ungleichheiten zwischen den Geschlechtern verschärft, den Zusammenbruch des Gemeinschaftsgefüges bewirkt und gleichzeitig bereits bestehende Gewaltketten verstärkt (Svampa 2017). In einem Rahmen, der durch die sozialen, beruflichen und räumlichen Merkmale der Enklave noch verschärft wird, wirkt sich dies in der Tat sehr negativ auf die Stellung der Frauen aus. Einerseits wird in einem Kontext ausgeprägter Lohnasymmetrien die traditionelle Rolle der Frau (MannArbeiter-Versorger/Frau-Hausfrau-Pflegerin) gestärkt. Ebenso erleben wir in vielen Ländern die Schwächung der gemeinschaftlichen und althergebrachten Rollen der Frauen, da die extraktiven Industrien das Gemeinschaftsgefüge aufbrechen und zu einer Verschiebung der Aktivitäten und sogar der Bevölkerung führen (Urgent Action Fund-Latin America 2017). Auf der anderen Seite wird der Prozess der sexuellen Ausbeutung von Frauen zentral und ihr Stellenwert als Sexualobjekt kristallisiert sich heraus. Schließlich nehmen in diesem Rahmen auch die Gewalthandlungen - sowohl körperliche als auch sexuelle - gegen Umweltaktivistinnen zu.

Die Konsolidierung der sozio-territorialen Konfigurationen, die durch Maskulinisierung, Auseinanderfallen des sozialen Gefüges,

8 Für einen Überblick über den Prozess der Kriminalisierung und Ermordung von weiblichen Kämpferinnen siehe Oxfam (2014). 
Ungleichheit und maximale und beschleunigte Überaneignung gekennzeichnet sind, verstärkt also die Matrix der patriarchalen Herrschaft und intensiviert die Gewaltketten. All dies äußert sich in einem schweren Rückschlag in Bezug auf die Gleichstellung der Geschlechter und einer sehr gefährlichen Wiederbelebung der schrecklichsten Figuren des Patriarchats und der Entstehung neuer Figuren, die mit der sexuellen Sklaverei verbunden sind.

\section{Die Expansion der Extremenergien und neue Konflikte}

Der technologische Fortschritt hat anderen Formen der Erdöl- und Erdgasförderung ermöglicht. Nicht-konventionelle Öl- und Gaslagerstätten sind technisch schwerer zu fördern, damit teurer in der Ausbeutung und mit größeren Kontaminationsrisiken verbunden. In Übereinstimmung mit der Definition von Tatiana Roa Avendaño von Censat-Agua Viva de Colombia und Hernán Scandizzo vom Observatorio del Petrolero Sur de Argentina soll in diesem Buch das Konzept der energías extremas (dt. Extremenergien) verwendet werden. Dieser Begriff ist weiter gefasst als der der nicht-konventionellen Kohlenwasserstoffe (also Erdöl und Erdgas) und bezieht sich "nicht nur auf die Eigenschaften der Kohlenwasserstoffe, sondern auch auf einen Kontext, in dem der Abbau von Erdgas, Rohöl und Kohle immer weitreichendere geologische, ökologische, arbeitsbezogene und soziale Risiken mit sich bringt; zusätzlich zu einer erhöhten Unfallrate im Vergleich zur traditionellen oder konventionellen Nutzung« (Roa und Scandizzo 2017).

Unter den Extremenergien finden sich verschiedene Typen: a) Schiefergas (shale gas), das in einer Tiefe zwischen 1000 und 5000 Metern existiert. Es ist in Schieferablagerungen und anstehendem Gestein, das sich aus Schlamm- und Tonablagerungen sowie aus anderem organischem Material bildet, zu finden. Schiefer ist sedimentäres Porengestein mit einer geringen Durchlässigkeit, da seine Poren sehr klein und nur schlecht miteinander verbunden sind. Typ b) ist das Tight Gas, das in einer kompakteren geologischen Struktur, etwa in Sand- oder Kalkstein, gefangen ist. Typ c) stellt das Kohlebodengas, 
auch bekannt als Coal Bed Methane (CBM), dar. Es taucht zusammen mit Kohlegestein in einer Tiefe zwischen 500 und 2000 Metern auf. Typ d) bezeichnet die zu den nicht-konventionellen Arten gehörenden schweren Rohöle oder bitominöse Kohlen, deren Folgen für die Umwelt ebenfalls sehr belastend sind. Derzeit wird dieser Typ in Kanada (Alberta) und im Orinoco-Gürtel (Venezuela) gefördert. Schließlich bezeichnet Typ e) Offshore-Erdölvorkommen, die in den Tiefen der Ozeane und zunehmend weiter von der Küste entfernt abgebaut werden, in einigen Fällen nach der Durchbohrung dicker Salzschichten. Die Tiefe, wie der Fall beim Pre-Salt-Verfahren in Brasilien zeigt, oder die Distanz zwischen der Meeresoberfläche und den Erdölreservoirs kann mehr als 7000 Meter erreichen.

Extremenergien sind mit hohen wirtschaftlichen Kosten sowie starken Auswirkungen auf die Umwelt und die Gesundheit verbunden. Ein Teil der Extremenergien muss anhand von Hydraulic Fracturing, auch bekannt als Fracking ${ }^{9}$, gewonnen werden. Es handelt sich dabei um eine experimentelle Technik, mit der es möglich ist, seit Jahrtausenden im Gestein eingeschlossenes Gas oder Rohöl zu extrahieren. Die Technik besteht darin, Wasser, Sand und Chemikalien unter hohem Druck in kohlenwasserstoffreiche Gesteinsformationen zu pressen, um deren Durchlässigkeit zu erhöhen und damit die Gewinnung der Rohstoffe zu verbessern. Die Expansion der Extremenergien ist eng mit den einseitig von den USA getroffenen geopolitischen Entscheidungen verbunden. Um die Jahrtausendwende setzten sich die Vereinigten Staaten

9 Obwohl sie schon seit längerem bekannt sind, wurden die nicht-konventionellen Kohlenwasserstoffe erst mit der Erweiterung der Crenzen des technologisch Möglichen und angesichts der bevorstehenden Erschöpfung der konventionellen Kohlenwasserstoffausbeutung als »gangbare« Alternative angesehen. Diese Entwicklung geschah trotz der höheren wirtschaftlichen Kosten, der größeren Umweltverschmutzung und -schäden sowie der geringeren Energieausbeute, die diese nicht-konventionellen Typen mit sich bringen. Ebenso wird die Technologie des Hydraulic Fracturing zwar seit mehr als 60 Jahren im Ölbereich eingesetzt, aber seit fast zwei Jahrzehnten intensiv und in großem Maßstab genutzt. Siehe Bertinat, D'Elia, Ochandio, Svampa und Viale (2014) und Svampa und Viale (2014). 
zum Ziel, ihre Abhängigkeit von den Golfstaaten zu beenden und Energieautarkie durch die Nutzung extremer Energien zu erreichen. Um eine tragfähige wirtschaftliche Bilanz zu erreichen, erhielten die Ölgesellschaften wichtige Zugeständnisse, die von starken Steueranreizen bis hin zu Befreiungen von Umweltauflagen reichten. Dies geschah unter der Präsidentschaft von George W. Bush im Jahr 2005, als das Parlament den Energy Policy Act (wegen der Lobbyarbeit des gleichnamigen Unternehmens auch als Halliburton-Amendment bekannt) verabschiedete. Das Gesetz befreit die Gasindustrie von der Einhaltung der Gesetze zum Schutz des Trinkwassers und anderer Umweltschutzbestimmungen und verhindert die Kontrolle der Aktivitäten durch die Environmental Protection Agency (EPA). Darüber hinaus sind die Unternehmen in den USA durch Vertraulichkeit geschützt und mussten bis vor kurzem nicht detailliert angeben, welche Chemikalien als Spaltflüssigkeiten verwendet wurden.

Diese von der Öllobby geförderte und aus geopolitischen Gründen gerechtfertigte Entscheidung führte zu einer Neugestaltung der globalen Energiekartographie auf der Basis der fossilen Energien. Infolgedessen haben die USA in den letzten zehn Jahren ihre führende Rolle als Nettoexporteur von Gas ausgebaut, und es heißt, dass sie bis Ende 2030 dank des Einsatzes von Fördertechnologien wie dem Fracking auch zum Nettoexporteur von Öl werden könnten. Russland und der Nahe Osten würden an Gewicht verlieren, ebenso wie China und andere Länder. Ein aktueller Bericht der Energy Information Administration (EIA) schätzt, dass die US-amerikanische Schieferölproduktion zwischen 2010 und 2025 um 8 Millionen Barrel steigen wird, was »die längste Periode anhaltenden Wachstums der Ölproduktion eines Landes in der Geschichte der Ölmärkte« wäre.

Im Jahr 2010 startete das US-Außenministerium die Global Gas Gap Initiative (GSGI), heute bekannt als Non-Conventional Gas Technical Commitment Program, das sich auf Hydraulic Fracturing konzentriert. Man lud mehrere Länder der Welt nach Washington mit dem Angebot ein, die Vorteile und Risiken dieser Technik zu diskutieren, die nach Ansicht ihrer Verteidiger den Energiemarkt grundlegend verändern würde. Im April 2011 veröffentliche die EIA einen Bericht zur Inwertsetzung 
und Verortung der weltweit größten Reserven. Obwohl diese Studie im Hinblick auf die (Über-)Schätzungen der Gasvorkommen in Frage gestellt wurde, wird sie immer noch als Grundlage für die Verteidigung der Gewährung des Zugangs zu diesen Reserven herangezogen. In dem Bericht werden die Gebiete mit den größten Vorkommen aufgezeigt, darunter China, die Vereinigten Staaten, Argentinien, Mexiko, Südafrika, Australien, Kanada, Libyen, Algerien und Brasilien. Während China und die USA beim nicht-konventionellen Erdgas mit 19,3\% bzw. 13\% führend sind, kommen Argentinien und Mexiko mit 11,7\% bzw. 10,3\% an dritter und vierter Stelle.

Somit verkörpert Argentinien den Brückenkopf des Frackings in der lateinamerikanischen Region. Im Jahr 2012, in einem Zusammenhang wachsender Energieknappheit, veranlassten die mehr als vielversprechenden Schätzungen über das Vorkommen nicht-konventioneller Kohlenwasserstoffe die Regierung von Christina Fernández, die staatliche Ölgesellschaft YPF teilweise zu enteignen, damals in den Händen der spanischen Firma Repsol. Unkonventionelle Kohlenwasserstoffvorkommen finden sich vor allem in Nordpatagonien, genauer im Neuquén-Becken, das insgesamt etwa $120.000 \mathrm{~km}^{2}$ umfasst. Über die Krise hinaus brach in Argentinien bald darauf ein eldoradistisches Fieber aus, das dazu beitrug, jede Diskussion über die Risiken des Frackings für die Umwelt, Gesellschaft und Gesundheit zu vermindern, wie dies auch in anderen Ländern der Fall war. Verstärkt wurde dies durch die nationalistische Rhetorik des kirchnerismo, die den Umstieg vom Commodity-Paradigma hin zum strategischen Ressourcenparadigma fördern sollte. Diese neue Leitlinie beruht auf der Kontrolle der Erdölund Erdgasförderung und damit auf der Energiepolitik durch den Staat.

Laut den Daten der EIA waren 2015 - ohne die Berücksichtigung der USA und Kanadas - Argentinien und China führend bei der Förderung von Schiefergas. Wie bereits bei der Sojaproduktion entwickelte sich Argentinien bei der Umsetzung einer der umstrittensten Fördertechniken zu einem Freiluftlabor auf globaler Ebene. Dies wurde durch einen zunehmend für ausländische Investitionen günstigen Regulierungsrahmen geschützt, insbesondere seit der Unterzeichnung des Ab- 
kommens zwischen YPF und Chevron. Das Abkommen stellte das Tor zum Fracking im großen Stil innerhalb des Landes dar und öffnete den Weg für weitere anschließende Mischverträge. Doch der progressistische kirchnerismo war mit dem Kurs auf Extremenergien nicht allein: Sowohl die politische Mitte als auch die rechte Opposition begrüßten die Entscheidung. Wieder einmal macht der Rohstoffkonsens, der Neuquén mit Vaca Muerta, der größten Schieferfelsformation des Landes, als das neue Saudi-Arabien projizierte, das Offensichtliche deutlich: Progressisten, Konservative und Neoliberale haben die gleiche Entwicklungsvision.

Dennoch verläuft diese historische Entwicklung nicht linear. Ab 2014 bremste der Rückgang der internationalen Ölpreise das Rohstofffieber in Vaca Muerta, was den Beginn eines Prozesses der sozialen und territorialen Umgestaltung aber nicht verhinderte. Diese Umgestaltung ging primär vom Bezirk Añelo aus, der von den großen transnationalen Konzernen besetzt wird. Es werden dort kaum noch Investitionen der globalen Megakonzerne erwartet, und alle sind jederzeit bereit dazu, im Fall eines Ausstiegssignals die Zelte (wieder) abzubrechen. In diesem Sinne machte sich die kirchneristische Regierung daran, die Ölproduktion zu subventionieren, was unter der Führung von Mauricio Macri fortgesetzt wurde. Macri brachte im Januar 2017 Vaca Muerta wieder in seiner neoliberalen Version eines Eldorados der Energiegewinnung auf den Weg. Er unterzeichnete Abkommen zur Garantie der Arbeitsflexibilität und verlagerte die Kosten der Akkumulation auf die schwächsten Sektoren in der Kette, also auf die Arbeiter.

Es ist hervorzuheben, dass es sich bei Vaca Muerta um alles andere als ein leeres Gebiet handelt, wie es von den provinziellen und staatlichen Behörden konzipiert wurde. Etwa 20 indigene Gemeinden sind über das Gebiet verstreut. Es handelt sich auch nicht um das einzige Gebiet Argentiniens, in dem Fracking gefördert wird: Auch im Alto Valle de Rio Negro in Allen, wo die Gewinnung von Tight Gas zwischen Birnen- und Apfelplantagen voranschreitet, schreitet das Fracking voran und droht diese Art der Landwirtschaft zu verdrängen.

Im Jahr 2014 sah sich die Regierung von Nequén dazu gedrungen, infolge der Proteste der Confederación Mapuche die seit 1927 im Ge- 
biet ansässige Gemeinde Campo Maripe anzuerkennen. Das strittige Gebiet beträgt laut dem Observatorio Petrolero Sur 10.000 Hektar, von denen die Regierung nur etwa 900 als Teil der Gemeinde offiziell anerkennt. In einem derart begrenzten Gebiet ist es unmöglich, den Bedarfen der ausgedehnten Weidewirtschaft und der Landwirtschaft nachzukommen. Beide sind die hauptsächlichen Tätigkeiten, die den dort lebenden 120 Menschen als Lebensbasis dienen. Dies ist nur ein Beispiel, und es gibt darüber hinaus noch viel intensiver umkämpfte Gebiete, die nun von den Mapuche-Gemeinschaften zurückerobert werden, die vor einer erweiterten geographischen Ausbreitung des Konflikts angesichts des Vormarschs der verschiedenen Formen des NeoExtraktivismus und der Landaneignung warnen.

Mit ähnlichen Argumenten wie in Argentinien hat die Regierung von Enrique Peña Nieto ab 2013 in Mexiko die Energiereform vorangetrieben, die die Tür für den Abschluss von Verträgen mit privaten Investoren öffnete und gleichzeitig die Frage der Gewinnung von Extremenergien in Schiefer- und Kompaktsandvorkommen auf die Agenda setzte, um dem Rückgang der Ölförderung und den wachsenden Erdgasimporten zu begegnen. An diesem Prozess sind mehrere Provinzen beteiligt, darunter Tampicas, Burgos, Veracruz, Burro und auch Chihuahua. Außerdem existieren Beweise für den bereits 2010 erfolgten Einsatz der Fracking-Methode in Schiefervorkommen durch die staatliche Firma Petróleos Mexicanos (PEMEX). Aktuell verfügt die FrackingIndustrie über 1.500 aktive Bohrlöcher. Neue Daten aus einer Untersuchung des Vereins CartoCrítica zeigen jedoch, dass 5.000 aktive Bohrlöcher vorhanden sind.

Mitte 2017 bereitete das kolumbianische Umweltministerium eine Regelung vor, die den Beginn der Offshore-Förderung im Land ermöglichen würde. Nichtsdestotrotz vertritt die Regierung keine einheitliche Position zum Fracking. Während das Umweltministerium vorschlug, die Studien zu den Auswirkungen des Hydraulic Fracturing um fünf Jahre zu verlängern, unterstützte das Energieministerium die sofortige Anwendung der Technologie. Die Alianza Colombiana Contra el Fracking (dt. Kolumbianische Allianz gegen Fracking) behauptet, dass diese Technologie, sofern die Grenze für Ölförderung ausgeweitet wird, 
mehrere strategisch wichtige Ökosysteme ernsthaft gefährden könnte. Darunter befinden sich der Sumapaz-Nationalpark, der die landwirtschaftliche Vorratskammer Bogotás ist und in dem sich außerdem das größte Moorland der Welt befindet. Auch das Moorland von Chingaza, dessen Wasserspeicher neben anderen Ökosystemen etwa $80 \%$ des Trinkwassers von Bogotá liefert, ist gefährdet.

In Brasilien förderte die Regierung von Michel Temer zeitgleich mit der zwischen 2016 und 2017 durchgeführten Energiereform Investitionen in die Erschließung und Ausbeutung neuer Erdöl- und Erdgaslagerstätten. Wie in anderen Ländern legte diese Reform fest, dass die staatliche Firma Petrobras Teil aller Ölkonsortien sein würde, die an der Gewinnung durch die so genannte Pre-Salt-Technologie beteiligt sind. Hierbei wird Erdöl in Tiefengewässern ausgebeutet. Diese Änderung machte jedoch die Reformen von 2010 rückgängig, die die nationale Ölindustrie gezwungen hatten, mindestens $30 \%$ der Erdölfelder in dieser Region zu erwerben (Pulso Energético 2017). Im Jahr 2017 betonte Fernando Coelho Filho, Minister für Bergbau und Energie, dass Brasilien in den kommenden Jahren erneut einen Moment der »Euphorie« mit der Pre-Salt-Technologie erleben würde. Eine ähnliche Stimmung herrschte damals unter der Regierungsführung des ehemaligen Präsidenten Luiz Inácio Lula da Silva mit der Entdeckung der großen Offshore-Reserven.

Der Vormarsch des Frackings hat zahlreiche Reaktionen der ansässigen Gemeinschaften auf dem ganzen Kontinent hervorgerufen. Der Widerstand in der Bevölkerung geht auf Bürgerversammlungen, indigene und bäuerliche Gemeinschaften, Umwelt-NGOs, Netzwerke von Intellektuellen und Akademikern und einige Gewerkschaften zurück. In Argentinien wurden ab 2012 zahlreiche Versammlungen und Bürgernetzwerke gegründet, die sich für einen Stopp und/oder ein Verbot der Ausbeutung von nicht-konventionellen Öl- und Gaslagerstätten durch die Frackingmethode einsetzen. Ende 2017 gab es bereits etwa 50 Standorte mit Verordnungen zum Verbot von Fracking. In Brasilien gab es im Jahr 201672 Städte, die das Hydraulic Fracturing verboten. Andere Daten weisen jedoch darauf hin, dass es 200 Gemeinden gab, die frei von Fracking waren, und mehrere Bezirke, die ein vollständiges Verbot 
in Erwägung ziehen. Es gibt viele brasilianische Aktivisten, die nach Argentinien reisen, um vor Ort die Schäden zu beobachten, die durch diese Art der Extremenergie entstehen, vor allem im oberen Rio Negro Tal, wo das Fracking zwischen Obstplantagen voranschreitet. Im gesamtregionalen Kontext wurde die Alianza Latinoamericana Contra el Fracking gegründet. Sie bildet ein Netzwerk von Organisationen, dessen Ziel es ist, die Debatte anzuregen, indem es den Energiekontext jedes Landes analysiert, und die öffentlichen Politiken, die zur Förderung und Regulierung des Frackings umgesetzt werden, betrachtet. Weitere Schwerpunkte des Netzwerks sind die sozialen, gesundheitlichen, ökologischen und wirtschaftlichen Auswirkungen auf die Bevölkerung, die Auswirkung des Fracking als Modell der territorialen Besetzung sowie die in jedem Land eingesetzten Strategien der Befürwortung, Mobilisierung und des Widerstands.

Das einzige Land in der lateinamerikanischen Region, das bisher einen Stopp des Frackings für vier Jahre beschlossen hat, ist Uruguay. Im August 2017 marschierten verschiedene Umweltgruppen aus Uruguay, Argentinien und Brasilien durch den Nordwesten des Landes, um gegen die Ausbeutung von Erdöl- und Erdgas durch die Frackingmethode $\mathrm{zu}$ demonstrieren und sich für den Schutz des Guarani-Aquifers, einer der größten Süßwasserreserven der Erde, einzusetzen. Schließlich wurde aus dem Vorhaben eines Stopps im Dezember 2017 ein gültiges Gesetz.

\section{Die Erweiterung der Geographie der Ressourcengewinnung}

Angesichts des Drucks, die Grenze für die Rohstoffgewinnung (insbesondere durch die Expansion von Extremenergien) zu erweitern, stellt sich die Frage, ob der Neo-Extraktivismus nicht auch eine Erscheinung ist, die heute für den globalen Norden gilt. Mit Sicherheit betreffen die Vertiefung des Neo-Extraktivismus und dessen Auftreten in extremer Form vor allem die südlichen Länder, indem sie ganze Landschaften umgestalten, neue Formen der Dominanz hervorbringen und die Geographie der Enteignung stärken. Dies geschieht in einem Kontext, der 
zunehmend von Verrechtlichung, staatlicher und parastaatlicher Repression sowie von patriarchalischer Gewalt geprägt ist. Jedoch geht der Druck zur Erweiterung der Grenzen für Energiegewinnung deutlich über die Länder des Südens hinaus und lässt das Vordringen einer extraktivistischen, territorialen Dynamik auch im globalen Norden erkennen. Ein berühmtes Beispiel ist die rasante Erweiterung der Grenze zur Gewinnung von Erdöl und Energie durch die Ausbeutung von nichtkonventionellem Gas und Öl. Die Bereitschaft zum Fracking impliziert in der Tat die Verstärkung der derzeitigen Energielandschaft, die auf fossilen Brennstoffen basiert, und bedeutet folglich einen starken Rückschritt in Bezug auf alternative Möglichkeiten oder den Übergang zu sauberen und erneuerbaren Energien. Es wurde bereits erwähnt, dass der Weg für das Fracking durch die Vereinigten Staaten im Namen der Selbstversorgung und der Energiesouveränität geebnet wurde. Die Geschichte seiner Entwicklung ab dem Jahr 2000 und die Reihe von ökologischen und wirtschaftlichen Ausnahmeregelungen, die es erforderte, sowie die entscheidende und mächtige Rolle der Öllobby gehören zu den schäbigsten Zeugnissen der jüngsten US-amerikanischen Politik. Infolgedessen hat das Fracking seit dem Jahr 2000 die Energielandschaft der Vereinigten Staaten verändert, da das Land nun über eine größere Autonomie in Bezug auf Importe verfügt. Gleichzeitig hat das Fracking es aber auch zu dem Gebiet gemacht, in dem die tatsächlichen Auswirkungen sichtbar sind: Unter anderem sind Grundwasserverschmutzung, gesundheitliche Schäden für Mensch und Tier, Erdbeben und erhöhte Emissionen von Methangas beobachtbar.

Der kontroverse Charakter des Frackings wird durch eine auffällige und mobile globale Verteilung des Konflikts veranschaulicht, der im Herzen des imperialistischen Nordens begann, wie das Verbot in Vermont und Moratorien in Staaten wie New York und der Metropolregion Los Angeles zeigen. Im Quebec (Kanada) führten die Widerstände zu einem Verbot, während sich in British Columbia nun infolge der $1.100 \mathrm{Ki}$ lometer langen Öl-Pipeline, die Bitumen aus der Region Alberta durch das Gebiet der ansässigen Gemeinden pumpt, ein indigen-urbaner Widerstand entwickelt. Im Fracking-Gebiet Alberta mit dem Epizentrum in der Stadt Fort McMurray, dem neuen Eldorado, sind die Unfälle und 
Schäden die durch das Fracking entstehen, unberechenbar. Es besteht die Möglichkeit, dass in der Folge eine menschenleere und trostlose Landschaft entsteht, die mehr als $90.000 \mathrm{~m}^{2}$ Land und Wasser umfasst und durch den Abbau von bituminösem Sand, dem schmutzigsten fossilen Brennstoff aller Extremenergien, verseucht ist. Seit dem Jahr 2000 hat die Erschließung dieses Gebiets Weltkonzerne wie Chevron, Total und Petrochina dorthin gelockt.

In Europa und anderen Regionen nährten die Berichte der EIA Erwartungen an ein neues Eldorado. Nicht wenige Länder ließen sich auf dieses verführerische Versprechen ein. Der vielleicht dramatischste Fall ist der Polens, in dem die US-amerikanischen Unternehmen bis 2011 Fuß fassten. Eine der wichtigsten Lobbyistinnen in dem Fall war Hillary Clinton, damals Funktionärin in der Obama-Regierung. Nachdem die US-Regierung vorhergesagt hatte, dass Polens Schiefergasreserven so reichlich vorhanden sein würden, um das Land für die nächsten drei Jahrhunderte mit Energie zu versorgen, war die Realität vier Jahre später eine andere: Die Kosten für die Förderung und die schlechte $\mathrm{Zu}$ gänglichkeit der Reserven ließen die Genehmigungen zurückgehen und Ölgesellschaften, darunter Chevron, verließen bald darauf das Land.

Frankreich verbot 2011 als erstes Land das Fracking. Der Kampf darum hatte den Widerstand verschiedener Kleinstädte in den Pyrenäen zusammengebracht und wurde vom wichtigen Referenten Jose Bové, Gesicht der globalisierungskritischen Bewegung in Frankreich, begleitet. Bulgarien sollte 2012 folgen, während sich andere Länder auf Moratorien beschränkten, wie etwa Deutschland. Letzteres gilt als eines der Länder, das sich am meisten für die Energiewende zu erneuerbaren Energien einsetzt. Fracking ist auch in Wales, Irland und Schottland verboten; die Schotten führten im Oktober 2017 eine Abstimmung durch, die ergab, dass $99 \%$ der Wähler »aus Gründen des Umweltschutzes und des mangelnden wirtschaftlichen Nutzens« gegen das Fracking waren. In Spanien ist der Konflikt zwischen Regierung und sozialem Widerstand nach wie vor sehr intensiv, und die Meinungskluft hat sogar den aktuell regierenden konservativen Partido Popular erreicht. In mehreren Gemeinschaften, darunter in Kantabrien und im Baskenland, unterstützten Abgeordnete des 
Partido Popular autonome Anti-Fracking-Gesetze. Angesichts einer Mehrparteien-Initiative zum Verbot des Frackings haben sich mehrere der Ölgesellschaften, die an der Förderung unkonventioneller Kohlenwasserstoffe in Spanien interessiert waren, für einen Rückzug bis 2016 entschieden.

Ein sehr konfliktträchtiges Land ist England. Nach Angaben des British Geological Survey (BGS) sind in Großbritannien Schiefergasvorkommen vorhanden, die das Land 25 Jahre lang versorgen könnten. Obwohl die ersten Bohrungen für Schieferöl und -gas im Jahr 2011 stattfanden, wurden sie mit Erdbebenwellen in Blackpool in Verbindung gebracht, was zu einem ersten nationalen Moratorium führte. Der Aufschub wurde jedoch einige Zeit später von der Regierung unter David Cameron aufgehoben. Cameron versprach den Gemeinden, die Fracking akzeptierten, Steuervorteile und schlug sogar vor, in Naturschutzgebiete vorzudringen. Auf die gleiche Art, wenn auch zurückhaltender, ist die Vorgehensweise der britischen Regierung nicht weit entfernt von der verleugnenden Haltung Donald Trumps. So beschloss die amtierende Premierministerin Theresa May, Fracking und Kernenergie einen Aufschwung zu geben und gleichzeitig das so genannte Department of Energy and Climate Change zu schließen.

So hat die Änderung der Spielregeln durch die USA auf der Suche nach Energieunabhängigkeit das globale Spielbrett neu konfiguriert, indem sie eine vertiefende Spielart des fossilen Energiemodells förderten. Dies geschah in einem derartigen Ausmaß, dass nicht einmal die Europäische Union dem Sirenenton des Frackings entkommen konnte. Viele Europäer zögerten nicht, angesichts des Versprechens der Energieunabhängigkeit auf die Freigabe ihrer Gebiete zu drängen. In vielen dieser Länder stießen die verschiedenen Regierungen und die Öllobby jedoch auf unerwarteten Widerstand in der Gesellschaft, der sie dazu zwang, das Kosten-Nutzen-Verhältnis nicht nur in wirtschaftlicher, sondern auch in politischer und sozialer Hinsicht zu überdenken.

Zusammenfassend lässt sich sagen, dass, während sich im Süden der soziale Widerstand proportional zur Geographie der Gewinnung ausdehnt, im Norden neue Konflikte um Naturressourcen entstehen. In einigen Fällen zeigen diese den wachsenden Protagonismus indige- 
ner Völker, wie z.B. in Kanada und den Vereinigten Staaten. In anderen Fällen zeigen sie die Reaktionsfähigkeit kleiner lokaler Gemeinschaften, wie unter anderem in England und in Frankreich. Folglich ist der NeoExtraktivismus im Energiebereich nicht nur in den Ländern der Peripherie anzutreffen. Trotzdem ist es unbestreitbar, dass er eine koloniale Dimension besitzt, wie der Fall Lateinamerika paradigmatisch zeigt. Die Neukonfiguration der klassischen neokolonialen Verteilung zwingt uns, die Beziehungen zwischen Nord und Süd unter einer Politik, die die Ausbreitung extremer Energien fördert, aus einer komplexeren Perspektive zu analysieren. Dies soll nicht heißen, dass sich die Asymmetrien zwischen zentralen und peripheren Ländern, also zwischen Nord und Süd, nicht ausgedehnt haben. Vielmehr führt uns die neue geopolitische Konfiguration dazu, das im Kern zivilisatorische Problem zu überdenken und so gleichzeitig die Möglichkeit zu erhöhen, im Kontext der neuen Kartierung des Widerstands Brücken und Bindungen der Solidarität auf globaler Ebene zu schaffen. 



\section{Kapitel 5: Ende des progressiven Zyklus und neue Abhängigkeiten}

In diesem Kapitel soll der geopolitische Kontext des Neo-Extraktivismus untersucht werden, beginnend mit dem globalen Aufstieg Chinas und der Intensivierung des Handelsverkehrs mit Lateinamerika. Genauer betrachtet werden die Formen, die die neue Abhängigkeit in Bezug auf China angesichts einer unvollständigen lateinamerikanischen Integration annimmt. In anderen Worten: Der Ansatz der progressistischen Regierungen $\mathrm{zu}$ einem vermeintlich unabhängigen Regionalismus ist gescheitert. In diesem Sinne liefern die beiden letzten Abschnitte dieses Kapitels eine Reflexion über die Grenzen des progressiven Wirtschaftszyklus.

\section{China und der Rahmen der neuen Unabhängigkeit}

In den letzten Jahren hat sich der Handelsaustausch zwischen Lateinamerika und China erheblich intensiviert. Bis zur Jahrtausendwende hatte China keine privilegierte Position als Ziel- oder Herkunftsland für lateinamerikanische Ex- und Importe. Bis 2013 war China jedoch bereits das wichtigste Herkunftsland für Importe nach Brasilien, Paraguay und Uruguay; das zweitwichtigste für Argentinien, Chile, Kolumbien, Costa Rica, Ecuador, Honduras, Mexiko, Panama, Peru und Venezuela; das drittwichtigste für Bolivien, Nicaragua, El Salvador und Guatemala. Auf diese Weise drängte China die Vereinigten Staaten, die Länder der Europäischen Union und Japan als Handelspartner zurück. 
Bei den Exporten ist China das erste Ziel von Brasilien und Chile, das zweite von Argentinien, Kolumbien, Peru, Uruguay und Venezuela (Svampa und Slivak 2016). Dieser Austausch ist jedoch asymmetrisch. Während es sich bei 84 \% der Exporte aus lateinamerikanischen Ländern nach China um Commodities handelt, sind 63,4 \% der chinesischen Exporte in die Region gewerbliche Industrieprodukte. Zur Veranschaulichung sollen ein paar Fälle genannt werden: Argentinien exportiert hauptsächlich Sojabohnen, Hülsenfrüchte und Pflanzenöle; Chile Kupfer; Brasilien Sojabohnen und Eisenerz; Venezuela und Ecuador Erdöl; Peru Eisenerz und andere Mineralien (Svampa und Slipak 2016). Selbst das Verhältnis zwischen einem Land wie Brasilien und China ist asymmetrisch und wird, wie bereits erwähnt, im Sinne einer »frühen Deindustrialisierung « interpretiert. Die Regierungen sind nicht dazu in der Lage, den Auswirkungen der so genannten Holländischen Krankheit, d.h. dem massiven Export von Rohstoffen, der mit der Ausbeutung der Naturressourcen verbunden ist, entgegenzuwirken (Salama 2011).

China hat bereits drei Freihandelsabkommen mit Ländern aus der Region - Chile (2005), Peru (2008) und Costa Rica (2011) - unterzeichnet. Die Unterzeichnung eines Abkommens mit Kolumbien steht kurz bevor. Nach fast zehn Jahren der Unterzeichnung zwischen Chile und China haben sich die Exporte des südamerikanischen Staates dorthin praktisch vervierfacht. Jedoch zeigt ihre Zusammensetzung einen sich vertiefenden Trend zur Beschränkung auf Primärgüter (Kupfer und seine Derivate, Eisenerz, Holz, weitere Mineralien und Früchte) (Svampa und Slipak 2016). Auch die Präsenz chinesischen Kapitals wird in der Region immer wichtiger. Einige Beispiele mögen dies verdeutlichen. Im Erdöl- und Erdgassektor sind die folgenden vier großen chinesischen Unternehmen vertreten: Sinopec, die China National Petroleum Corporation (CNPC), die China National Offshore Oil Company (CNOOC) und Sinochem. Bis 2010 waren diese vier Firmen bereits an rund $15 \mathrm{Ab}$ bauprojekten in Peru, Venezuela, Ecuador, Kolumbien, Brasilien und Argentinien beteiligt.

Was den Kohlebergbau und Mineralien betrifft war das Hauptziel chinesischer Investoren schon immer Peru, gefolgt von Brasilien und neuerdings Ecuador. Die aktivsten Unternehmen sind Minmetals und 
Chinalco. Im Jahr 2014 erwarb Minmetals die peruanische Mine von Las Bambas - eine der größten Kupferförderstätten weltweit - von Glencore-Xstrata. In Ecuador gewährte die Regierung von Rafael Correa der Firma Ecuacorriente (Ecsa), deren Aktionäre die chinesischen Aktiengesellschaften Tongling Nonferrous Metals Group Holdings und China Railway Construction Corporation Ltd. sind, die Ausbeutung der Projekte San Carlos Panantza und Mirador. Mit diesen Konzessionen sollte der chinesische Staat mehr als die Hälfte des ecuadorianischen Kupferabbaus und mindestens ein Drittel des Gold- und Silberabbaus kontrollieren (Chicaiza 2014). Laut Acción Ecológica wurden 2012 chinesische Unternehmen, die mit dem Bergbauprojekt Mirador in Verbindung stehen, wegen Nichteinhaltung von Arbeitsstandards, Misshandlungen, ungerechten Löhnen und Unfällen durch die indigene Gemeinschaft der Shuar angezeigt. 2016 kam es zu neuen Konflikten, als die Shuar ein Bergbaugebiet im Amazonas besetzten. Die Ansiedlung des chinesischen Unternehmens erfolgte ohne eine freie, informierte Konsultation und durch eine Militarisierung der Gebiete. ${ }^{1}$ Ebenso lässt sich anführen, dass das chinesische Unternehmen Shandong Gold $50 \%$ des Veladero-Projekts zur Ausbeutung von Gold in der argentinischen Provinz San Juan erworben hat, das für zwei bedeutende Zyanidaustritte 2015 und 2016 verantwortlich ist, schon bevor es mit dem kanadischen Unternehmen Barrick Gold in Verbindung stand.

Ein weiteres Thema, das an Relevanz gewinnt, ist die Kreditvergabe. Jüngste Untersuchungen zeigen, dass die Mehrzahl der chinesischen Darlehen in der Region für die Infrastruktur (55\%), die Energiewirtschaft (27\%) und den Bergbau (13\%) bestimmt waren. Hauptkreditgeber war die China Development Bank, die rund $71 \%$ der chinesischen

Im Dezember 2016 eskalierte der Konflikt in Folge der Forderungen der Shuar so stark, dass es einen Toten und mehrere Verletzte gab. Die Reaktion des damaligen Präsidenten Correa bestand darin, den nationalen Ausnahmezustand auszurufen, die Shuar als "paramilitärische und halbkriminelle Gruppen« zu bezeichnen und die Auflösung von Acción Ecológica bekanntzugeben. Angesichts der nationalen und internationalen Solidaritätsbekundungen lehnte das ecuadorianische Umweltministerium den Auslösungsantrag des Innenministeriums letztlich $a b$. 
Darlehen an die Region vergab, und der Hauptkreditnehmer Venezuela, der etwas mehr als die Hälfte dieser Darlehen zur Finanzierung von 13 Projekten erhielt. Chinesische Kredite an Ecuador und Venezuela ersetzen zunehmend die Ausgabe von Staatsanleihen dieser Länder und werden mit Öl oder einem anderen Primärgut (auch rohstoffgebundene Kredite genannt) garantiert. Dies beinhaltet eine Investitionspolitik mit der Beteiligung chinesischer Unternehmen. ${ }^{2}$

Auf der anderen Seite stellt sich die Frage, was der Zweck der chinesischen Investitionen ist. Diesbezüglich ist man sich in den Nachforschungen einig: Die Investitionen sind vor allem in der Rohstoffindustrie (Bergbau, Erdöl, Agrarindustrie, Mega-Staudämme) angesiedelt, was den repressiven Effekt, den die Volkswirtschaften unter dem Rohstoffkonsens erleben, noch verstärkt. In einigen Fällen sind die chinesischen Investitionen auch auf den tertiären Sektor ausgerichtet, dann aber um den Primärsektor zu unterstützen. Dieser Ansatz bedeutet sogar eine Bedrohung für die von kleinen und mittelständischen Unternehmen (KMU) gebildeten ökonomischen Cluster in der Region, sowohl wegen der grassierenden Umweltverschmutzung als auch wegen der Tendenz, Rohstoffe direkt nach China zu exportieren, die zuvor noch von lokalen KMU verarbeitet wurden.

$\mathrm{Zu}$ Beginn des Rohstoffkonsenses und im Klima des Aufstiegs progressiver Regierungen sahen die meisten Analysten und Politiker die anfänglichen Beziehungen zwischen Lateinamerika und China positiv. Sie folgten der Argumentation, dass die ökonomischen Verbindungen zu China die Autonomie der Region gegenüber der Hegemonie der USA stärken könnte. Es war der Ex-Präsident Venezuelas Hugo Chávez, der diese Art der Positionierung anführte und eine Politik der offenkundigen Annäherung an China verfolgte. Aufgrund des Ölreichtums sah Chávez China als den idealen kommerziellen und politischen Verbündeten, um sich von den Vereinigten Staaten zu distanzieren. In diesem Kontext zeigt sich ein Szenario des beschleunigten Übergangs von genüber China auf rund 70 Milliarden US-Dollar. Das Land zahlte seine Auslandsschulden mit 600.000 Barrel Öl pro Tag zurück. 
einer bi- zu einer multipolaren Weltordnung, wobei den Verbindungen zu China mit Blick auf die geopolitischen Kräfteverhältnisse in Lateinamerika ein politischer Charakter innewohnt. Aus optimistischer Sicht eröffnen die Handelsbeziehungen mit China die Möglichkeit einer Süd-Süd-Kooperation zwischen »aufstrebenden Entwicklungsländern«. Jenseits des Etiketts des "Schwellenlands« und der Schwierigkeit, die Selbstdarstellung Chinas als aufstrebendes Land zu akzeptieren, wird jedoch deutlich, dass der kometenhafte globale Aufstieg des asiatischen Landes sowie die Realpolitik der Handelsbeziehungen mit Lateinamerika weit davon entfernt sind, die Hypothese eines ausgeglichenen Süd-Süd-Verhältnisses zu verwirklichen.

Im Laufe des progressiven Zyklus hat die Entwicklung der Beziehungen zwischen China und den verschiedenen lateinamerikanischen Ländern die Vorstellung einer Süd-Süd-Kooperation geschwächt. Ebenso wurde die Hypothese eines antihegemonialen Regionalismus durch die Verabschiedung einer Union Südamerikanischer Nationen (UNASUR) mit "geringer Stärke« relativiert (Comini und Frenkel 2014), gekennzeichnet durch das Ende der großen politischen Führungspersönlichkeiten der Region: Mit dem Tod Hugo Chávez' und Nestor Kirchners sowie die Verhaftung Lula da Silvas sind drei Politiker abgetreten, die stark auf die regionale Integration Lateinamerikas gesetzt hatten.

Eine weitere strittige Angelegenheit ist die Bewertung des Ausmaßes des lateinamerikanischen Regionalismus. Es sei daran erinnert, dass einer der wichtigsten Wendepunkte dieses neuen Regionalismus der IV. Amerika-Gipfel in Mar del Plata (Argentinien) im Jahr 2005 war, der von den Vereinigten Staaten vorangetrieben wurde. Anschließend wurde die Bolivarianische Allianz für die Völker unseres Amerika (ALBA) unter dem Anstoß des charismatischen Hugo Chávez gegründet. In einer klar lateinamerikanisch geprägten Linie wurden ehrgeizige Projekte geboren, darunter jenes der Schaffung einer eigenen Währung (der ecuadorianische Sucre) und die Bank des Südens, die jedoch beide nicht erfolgreich waren. Dies ist zum Teil auf die mangelnde Bereitschaft Brasiliens zurückzuführen, einem Land, das aufgrund seiner Rolle als aufstrebende Macht bereits in anderen globalen Ligen spielt. 
Die Gründung der UNASUR im Jahr 2007 und später der Gemeinschaft der Lateinamerikanischen und Karibischen Staaten (CELAC) im Jahr 2010, zunächst als Forum der Konfliktaufarbeitung und ohne die USA, setzten Eckpunkte der regionalen Integration. All dies hinderte die Vereinigten Staaten jedoch bei weitem nicht daran, später mit mehreren lateinamerikanischen Ländern bilaterale Freihandelsabkommen zu unterzeichnen. 2011 wurde ein neuer regionaler Block, bestehend aus der Pazifik-Allianz, unter Beteiligung von Ländern wie Chile, Kolumbien, Peru und Mexiko geschaffen.

Sowohl die Idee des neuen antihegemonialen Regionalismus als auch die einer Süd-Süd-Kooperation mit China scheinen eher mit einer Art Wunschdenken zu tun zu haben als mit den real existierenden Wirtschafts- und Handelspraktiken der verschiedenen progressiven Regierungen. In der Tat ist die Unterzeichnung einseitiger Abkommen mit dem asiatischen Riesen durch die lateinamerikanischen Regierungen (von denen viele ihre Wirtschaft auf Jahrzehnte vertraglich verpflichten) keineswegs die Ausnahme. Im Gegenteil, sie sind in der letzten Zeit eine ziemlich weitverbreitete Regel, die, anstatt die lateinamerikanische Integration zu stärken, den Wettbewerb zwischen den Ländern als Rohstoffexporteure nur verstärkt.

Es stimmt zwar, dass das Vordringen und die rasche Konsolidierung des chinesischen Einflusses in Lateinamerika als Chance für eine größere Autonomie gegenüber den Vereinigten Staaten gesehen wurden. Dennoch führten der eher rhetorische lateinamerikanische Regionalismus, die unilateralen Verhandlungen mit China, die faktische Konkurrenz zwischen den verschiedenen Ländern der Region und der Anstieg der Rohstoffexporte zu einer Verstärkung der Ungleichheiten und konfigurierten die Vertiefung eines Extraktivismus mit neuen $\mathrm{Ab}$ hängigkeiten. Der bemerkenswerteste Aspekt ist also nicht die - übrigens unvermeidliche und notwendige - Verknüpfung Lateinamerikas mit China, sondern die Art und Weise, in der sie auf der Basis der Nachfrage nach Rohstoffen und des ungleichen Austauschs funktioniert. In Wirklichkeit bedeutet diese Beziehung die Stärkung des Extraktivismus und die Unterdrückung der lateinamerikanischen Volkswirtschaf- 
ten vor dem Hintergrund des Rückgangs eines starken lateinamerikanischen Regionalismus.

\section{Das Ende des progressiven Zyklus als lingua franca}

Das Auftreten verschiedener progressiver Regierungen weckte in der Bevölkerung hohe Erwartungen an die Politik. Im Klima des Rohstoffkonsenses stellte der Progressivismus eine Art lingua franca dar, d.h. ein kollektiver Rahmen, der in der Lage war, die politischen Differenzen durch eine gemeinsame Sprache zu vereinheitlichen. Gleichzeitig ordnete oder hierarchisierte er sie, indem er eine Art Abstufung von den politisch radikaleren Ländern (v.a. die Bolivarianische Achse, die durch Venezuela, Bolivien und Ecuador dargestellt wird) zu den gemäBigteren (u.a. Brasilien, Argentinien, Uruguay) herstellte. Die zentralen Elemente, die diese lingua franca kennzeichneten, waren die Infragestellung des Neoliberalismus, eine heterodoxe Wirtschaftspolitik, die Ausweitung der Sozialausgaben und schließlich das Bestreben, einen lateinamerikanischen Raum zu schaffen, von dem aus über die Perspektive der regionalen Integration nachgedacht werden kann. Zweifelsfrei war die Konsolidierung einer progressiven politischen Hegemonie, die mit diesen modularen Elementen verbunden ist, mit dem Boom der internationalen Rohstoffpreise verbunden. Dies wird hier sowohl in wirtschaftlicher als auch in politischer und sozialer Hinsicht als Rohstoffkonsens charakterisiert.

Während des progressiven Zyklus (2000-2015) gab es Stimmen, die dazu neigten, mehr oder weniger automatisch Progressive und Linke gleichzusetzen. Auf nationaler und regionaler Ebene war jedoch das, was unter Progressivismus verstanden wurde, Gegenstand harter Debatten und Interpretationen. Dies bezieht sich insbesondere auf Fragen, die unter anderem mit der Konzeption des sozialen Wandels, der Verbindung zu sozialen Bewegungen und der Ausbreitung des Neo-Extraktivismus zusammenhängen. Diese Tendenzen zeigten die wachsende Spannung zwischen verschiedenen dekolonisierenden politischen Narrativen, vor allem zwischen dem national-entwick- 
lungsorientierten und dem indigenistischen Narrativ, die eine große Rolle bei den Veränderungen ab der Jahrtausendwende spielten, allem voran beim Infragestellen der neoliberalen Hegemonie und der Öffnung eines neuen politischen Szenarios. Einerseits wurde das entwicklungspropagierende Narrativ, das durch den aufstrebenden Neo-Extraktivismus fortgeschrieben wurde, besonders im Zusammenhang mit der tief in der Region verwurzelten Tradition des Populismus neu artikuliert. Andererseits wurde das indianistische Narrativ - durch die sozialen Kämpfe gegen den Neo-Extraktivismus neu orientiert über den Umwelt- und Autonomiediskurs aktualisiert. Dabei traten gegen Ende des Zyklus unter anderem populäre Feminismen in Erscheinung, die das hervorbrachten, was als ökoterritoriale Wende der Kämpfe bezeichnet wird.

Dennoch ist es wichtig, die Abstufungen und Nuancen der jeweiligen nationalen Kontexte im Blick zu behalten. Trotz der Konsolidierung des Neo-Extraktivismus als Entwicklungsstrategie und dem explosionsartigen Anstieg sozio-ökologischer Konflikte wurden in einigen Ländern die Auseinandersetzungen zwischen den verschiedenen Lagern nicht mit gleicher Intensität geführt oder hatten nicht die gleiche öffentliche Sichtbarkeit. In Brasilien und Chile erscheint das ökologische Narrativ, das hier grundsätzlich gemeinschaftlich geprägt ist, primär in Verbindung mit einer Reihe kaum wahrnehmbarer und verstreuter, lokal isolierter Stimmen, die von den am weitesten am Rand stehenden Gruppen kommen (Indigene, Bauern, kleinere und mittlere Lokalversammlungen). In Bolivien und Ecuador hingegen erlangten Darlegungen der indigenen Protagonisten und der Umweltorganisationen beachtliche Bekanntheit und Relevanz in der öffentlichen Agenda. Ihre Verknüpfung mit den in den neuen politischen Verfassungen enthaltenen horizontalen Konzepten, wie dem buen vivir, der Plurinationalität und den Naturrechten, hatte diesen Organisationen eine herkunftsbasierte Legitimierung verliehen, die durch den aufsteigenden Populismus jedoch allmählich in Frage gestellt wurde.

Diese Entwicklung zeichnete sich durch eine Verschärfung der Konfrontation verschiedener politischer Narrative im Laufe des progressiven Zyklus aus. Dies fand nicht nur im Kontext der Kämpfe gegen den 
Neo-Extraktivismus und der wachsenden Kriminalisierung des sozioökologischen Widerstands statt, sondern war auch eine Folge der politischen und sozio-ökonomischen Unzulänglichkeiten und Limitierungen der progressiven Regierungen selbst. Die herausgehobene Betonung von Rechten, die Reduktion von Armut und die Politik der sozialen Inklusion, die Erhöhung der Löhne und die Steigerung des Konsums standen während der Jahre des Commodity-Booms neben einer Strategie der Unterwerfung und des Entzugs der Autonomie der sozialen Organisationen und Bewegungen. Dies ging einher mit einer wachsenden Dynamik der Personalisierung der politischen Macht sowie mit dem Fortbestehen von Ungleichheiten und politischen Kompromissen, die immer häufiger im extraktiven Sektor sichtbar wurden, nicht zuletzt unter einem bemerkenswerten Einfluss des transnationalen Kapitals. Die Transformation der ländlichen Welt zeichnete sich dabei im Besonderen durch einen beschleunigten Prozess der Landnahme aus.

Im Endeffekt setzt sich eines der beiden gegnerischen Narrative als das dominante durch, nämlich das populistisch-entwicklungsorientierte. Dabei gestaltete es sich als tendenziell exklusiv, wobei es jedoch bestimmte Elemente aus anderen politischen Matrizen absorbierte und umdeutete (wie etwa der der klassenorientierten und autonomen Linken). Gleichzeitig schloss das populistisch-entwicklungsorientierte Narrativ andere politische Perspektiven (verbunden mit den comunitaristas und ökologischen Kreisen) aus, die schwieriger und lästiger $\mathrm{zu}$ integrieren gewesen wären. Die Diskussion darüber, was als »links« angesehen wurde, ist im lateinamerikanischen Raum hiernach heftiger geworden. Es ist Tatsache, dass gegen Ende des progressiven Zyklus (2015-2016) rekursive politische Prozesse und soziale Dynamiken durch die Entkoppelung zwischen Progressiven und Linken eloquenter wurden. In einigen Fällen - wie dem der Arbeiterpartei PT in Brasilien könnte man, wie Modonesi hervorhebt, von einer "genetischen Mutation« (Transformismo) im politischen Kontext sprechen; in anderen sieht man die Entwicklung hin zu traditionelleren Herrschaftsmodellen, die in einer bestimmten politischen Tradition verankert sind (starker Populismus, Svampa 2016). Jedenfalls würde man in beiden Fällen von einer sogenannten »konservativen Modernisierung« sprechen (u.a. Schavel- 
zon 2016; Singer 2012). Sogar das feste Vorhaben, einen mächtigen und antihegemonialen lateinamerikanischen Raum zu institutionalisieren, blieb unvollendet. Vergleicht man den IV. Amerika-Gipfel in Mar del Plata (Argentinien) im Jahr 2005 mit der Lateinamerikanischen Freihandelsvereinigung ALALC ein Jahrzehnt später, so wird klar, dass ersterer bereits der Höhepunkt des lateinamerikanischen Regionalismus war, obwohl er eigentlich der Ausgangspunkt einer neuen lateinamerikanischen Zusammenarbeit hätte sein sollen. Dieser Regionalismus hätte grundsätzlich und authentisch integrativ wirken sollen, ausgerichtet auf die Schaffung einer Plattform von regionaler Reichweite und mit der Fähigkeit, mit neuen und mächtigen Handelspartnern, darunter China, zu verhandeln.

Außerdem setzten die Progressiven (Populisten wie Transformisten) nach außen hin einen Akzent auf den ideologischen Kampf mit verschiedenen Machtgruppen, insbesondere mit den Massenmedien. Die lateinamerikanischen Populisten des 20. Jahrhunderts wurden traditionell mit der Leitidee des Sozialpakts assoziiert, auch wenn sie diesen entlang einer Art Kriegsrhetorik durchzusetzen suchten. Die Progressiven des 21. Jahrhunderts stellten ein ähnliches Schema auf, d.h. sie stellen einerseits den Neoliberalismus in Frage, aber anderseits paktierten sie mit dem Großkapital. Trotzdem - oder gerade deswegen gerieten sie bald in eine große politisch-ideologische Konfrontation mit rechten Strömungen, die über die Massenmedien ausgetragen wurde.

So vereinfachte die Betonung der Polarisierung auch je nach Fall und auf unterschiedliche Weise $\mathrm{zu}$ unterschiedlichen Momenten die Wahlmöglichkeiten und teilte das politische Feld zwischen zwei antagonistischen Blöcken auf: auf der einen Seite die progressiven Kräfte, die sich die Repräsentation des Volkswillens zum Ziel setzten, auf der anderen Seite die verschiedenen Parteien oder Koalitionen der Rechten, die sich der vermeintlichen Verteidigung der Republik verschrieben. Diese Vereinfachung des politischen Raums führte bei den Progressiven zur Stärkung von Verschwörungstheorien: Am Ende waren der Imperialismus, die allgegenwärtigen Rechten oder die Massenmedien die Schuldigen; außerdem war von diesem Standpunkt aus jegliche Kritik an den Progressiven, die von der ökologischen, kommuni- 
tären oder klassenkämpferischen Linken angebracht wurde, am Ende »funktional« für die Logik der am stärksten konzentrierten Sektoren. Auf der Seite der rechten Kreise führte diese Opposition zur Verteufelung der verschiedenen progressiven Erfahrungen, die zur Mitte bzw. zum Ende des Zyklus hin als »unverantwortlicher Populismus« bezeichnet wurden. Sie waren schuldig, die mit dem Rohstoffboom verbundene Zeit der wirtschaftlichen Prosperität verschwendet und auf eine reine Matrix der Korruption reduziert zu haben. Dabei könnten die rechten Kreise auch auf ihre Intellektuellen, ihre rettenden Reden und die Unterstützung und/oder Förderung großer Medien zählen.

Die politische Überreaktion der Progressiven - die an einem Punkt versuchten, die Idee zu etablieren, dass sie und nur sie den Volkswillen verkörpern könnten - wurde durch die wachsende Wirtschaftskrise und Korruptionsskandale verstärkt und führte dazu, dass die politische Bühne zunehmend von Spaltung und Konfrontation geprägt war, auf der alleine die Möglichkeit des Regierungswechsels in Folge von Wahlen mit großen Drama aufgenommen wurde. Dies ist seit Jahren in Venezuela der Fall, wo die Krisensituation tiefgreifend ist, aber die Regierung Maduros sich trotz aller Widerstände nach seiner Wiederwahl im Mai $2018 \mathrm{zu}$ festigen scheint. In Argentinien siegte 2015 schließlich die Rechte, die zwei Jahre danach bei den Zwischenwahlen wieder bestätigt wurde; ebenso in Ecuador, wo sich Lenin Moreno 2017 äußerst knapp gegen den rechten Kandidaten durchsetzte, um sich dann rasch von seinem Vorgänger Rafael Correa zu distanzieren und mit diesem $\mathrm{zu}$ brechen. Spaltung und Polarisierung wurden selbst im Bolivien des Evo Morales, einem der erfolgreichsten Präsidenten dieser Zeit, offensichtlich. Unter anderem war dies mit seinem hartnäckigen Willen, das Referendum vom Februar $2016 \mathrm{zu}$ ignorieren, verbunden. Es hinderte ihn daran, zum vierten Mal in Folge für die Präsidentschaft zu kandidieren. Am Ende wurde jedoch die Justiz so manipuliert, dass sie eine solche Möglichkeit doch zuließ.

Trotz der Überreaktionen handelt es sich nicht ausschließlich um konspirative Thesen, denn die Prozesse der politischen Polarisierung ermöglichten den fadenscheinigsten Weg des parlamentarischen Putsches, wie der frühe Sturz von José Manuel Zelaya in Honduras (2009), 
die rasche Amtsenthebung von Fernando Lugo in Paraguay (2012) und das Impeachment der brasilianischen Präsidentin Dilma Roussef (2016) zeigten, die alle die Rückkehr zu einem offen konservativen Umfeld in diesen Ländern beschleunigten.

\section{Die Grenzen des real existierenden Progressismus}

Die Erkämpfung einer progressiven Hegemonie war mit dem Wachstum der Volkswirtschaften und der Verringerung der Armut verbunden. In einem Bericht der Wirtschaftskommission für Lateinamerika und der Karibik (Cepal) aus 2012 wird der weltweite Rückgang der Armut (von $44 \%$ auf 31,4\%) zwischen 2001 und 2011 sowie der Rückgang der extremen Armut (von 19,4\% auf 12,3\%) gemeldet. Dies ist nicht nur auf die Erhöhung der Löhne, sondern auch auf den Ausbau einer Bonus- und Sozialplanpolitik (bedingte Transferprogramme) zurückzuführen. Im Einklang mit der Armutsbekämpfung zeigten die ersten, auf dem Gini-Koeffizienten basierenden Studien auch in Lateinamerika eine Verringerung der Ungleichheit zwischen 2002 und 2010. Vor einigen Jahren begannen jedoch mehrere Autoren, diese Aussagen zu relativieren, indem sie argumentierten, dass die verfügbaren Daten sich nur auf kurze Zeiträume beziehen und eine langfristige Betrachtung nicht zulassen. Zudem war die Verringerung der Einkommensungleichheit an eine Erhöhung der Löhne gebunden, nicht aber an eine Reform des Steuersystems, das sehr komplex, undurchsichtig und vor allem regressiv wurde (Salama 2015).

Andere Argumente sehen die Unterscheidung zwischen struktureller und konjunktureller Ungleichheit. Während in den 1990er Jahren Armut und Ungleichheit in der Region zunahmen, wurden im ersten Jahrzehnt des 21. Jahrhunderts beide auf dem gesamten Kontinent reduziert. Dies führt zu der Annahme, dass wir es mit einer Entwicklung zu tun haben, die unabhängig von den ideologischen Botschaften der Regierungen stattfindet und darauf hindeutet, dass wir es mit einem Trend $\mathrm{zu}$ tun haben, der durch strukturelle wirtschaftliche Faktoren verursacht wird, die mit der Eingliederung der Region in den Welt- 
markt zusammenhängen (Machado und Zibechi 2016). Hinzu kommt, dass es auch keine Steuerreform gab und die wirtschaftlichen Interessen der Eliten nicht berührt wurden. Das Steuersystem ist weiterhin regressiv; im Jahr 2013 machte die Steuer der reichsten Sektoren 3,5\% der gesamten Steuereinnahmen aus, während die Mehrwertsteuer (MwSt.) um ein Drittel auf $36 \%$ stieg und in vielen Ländern zur Hauptquelle der Steuererhebung wurde (Burchardt 2016, 69).

In neueren Studien wird argumentiert, dass die Senkung der Armut nicht zu einer Verringerung der Ungleichheiten geführt hat. Von den Studien Thomas Pikettys' inspirierte Untersuchungen, die sich auf die Superreichen konzentrieren und die Steuererklärungen der reichsten Bevölkerungsschichten analysieren, zeigen, dass in Ländern wie Argentinien, Chile und Kolumbien $1 \%$ der Bevölkerung zwischen $25 \%$ und $30 \%$ des Vermögens besitzt (Kessler 2016, 26). Andere Untersuchungen, die in Brasilien, einem der am meisten durch Ungleichheiten geprägten Länder der Region, durchgeführt wurden, stellen die angebliche Verringerung der Schere zwischen Arm und Reich zwischen 2006 und 2012 in Frage. Die Arbeit des Instituto de Pesquisa Econômica Aplicada (IPEA) zeigt eine Zunahme der Ungleichheiten, da im Jahr 2012 die reichsten $1 \%$ der Bevölkerung 24,4\% des nationalen Einkommens ausmachten, während dieser Prozentsatz 2006 noch 22,8 \% betrug. Unter den reichsten $10 \%$ stieg die Verteilung des nationalen Einkommens im gleichen Zeitraum von $51,1 \%$ auf 53,8 \% (Zibechi 2015). Obwohl also die extreme Armut in Brasilien reduziert wurde und der Konsum sich ausgeweitet hat, bestehen die Ungleichheiten weiter und nehmen sogar leicht $\mathrm{zu}$. Olaf Kaltmeier konstatiert in diesem Kontext eine neue Tendenz der Refeudalisierung (2020).

Kurz gesagt, die Progressiven paktierten mit dem Großkapital (also Rohstoff- und in einigen Fällen Finanzkapital), wobei sie ein Aufeinanderstoßen der Sektoren, insbesondere der Massenmedien, welche die politische und mediale Agenda bestimmen, in Kauf nahmen. Ebenso führten sie wenn überhaupt nur zaghafte Reformen des Steuersystems durch und beanspruchten die für die Konstituierung der gesamten Armutsrate günstige Erfassung außerordentlicher Einkommen für sich. Wie Stefan Peters hervorhebt, war der Neo-Extraktivismus eine Vor- 
aussetzung für die erfolgreiche Etablierung progressiver Regierungen, aber gleichzeitig auch eines der größten Hindernisse für die Verwirklichung tiefgehender und struktureller Reformen in der Region (Peters 2016, 22).

Das Ende des progressiven Zyklus bedeutet jedoch nicht das vollständige Ende der progressiven Regierungen. Uruguay und Bolivien halten an dieser politischen Linie fest. Ecuador steht zwischen fortschrittlicher progressiver Politik und dem politischen Abdriften nach rechts. Es ist unsicher, wie es in Mexiko, wo Andrés Manuel López Obrador die Präsidentschaftswahlen gewonnen hat, weitergeht. Tatsache aber ist, dass wir das Ende des Progressivismus als lingua franca erleben, jenseits des Fortbestehens solcher Regierungen und sogar der zu beobachtenden politischen Mutationen. Und dieses Szenario des Niedergangs konfrontiert uns mit einer harten Realität: Innerhalb der linken Strömungen ist das politische Panorama sehr kritisch. Die selektive Progressivität der lateinamerikanischen Regierungen hat im Umfeld kritischer sozialer Bewegungen tiefe Wunden hinterlassen, die nur schwer heilen. Dies ist etwa der Fall in Ecuador, wo einige Kreise der Confederación de Nacionalidades Indígenas del Ecuador (CONAIE) (dt. Konföderation indigener Nationen in Ecuador), die sich früher mit den linken Parteien identifizierten, bei den letzten Präsidentschaftswahlen für den Kandidaten der Rechten stimmten.

Die Erschöpfung und das Ende des progressiven Zyklus gibt keinen Anlass zum Feiern. Sicherlich bewegt es uns dazu, über die Dissoziation zwischen den in ihrer eigentlichen Form existierenden Regierungen nachzudenken sowie über die Entwicklung solcher Regime hin zu traditionellen Herrschaftsmodellen wie dem Populismus, dem Transformismus und der passiven Revolution. Der neue politische Kurs konfrontiert uns mit einem neuen Szenario: Mehr und mehr verabschiedet sich Lateinamerika von einer gemeinsamen Sprache. Stattdessen bestehen einige progressive Regierungen (mit all ihren politischen Mutationen) fort und es besteht sogar die Möglichkeit, dass andere Szenarien hinzukommen (wie z.B. in Mexiko). Die politische Bühne zeigt auch einen reformierten rechten Flügel, der ganz offen das neue Unternehmertum befürwortet (Argentinien und Brasilien). Tatsächlich gibt es dort, wo 
es einen Machtwechsel gab, Kontinuitäten und Brüche im progressiven Zyklus. Ersteres ist mit der Stärkung der aktuellen Rohstoffindustrien verbunden, letzteres mit einem offenen Rückgang der zuvor erkämpften sozialen Rechte. Diese Kontinuitäten und Brüche finden in einem Rahmen statt, der die Achtung der Freiheit und der Grundrechte der am meisten benachteiligten Bevölkerungsgruppen immer mehr in Frage stellt. Dies eröffnet ein neues Bild auf globaler und regionaler Ebene, das stärker zersplittert und weniger vorhersehbar ist und das Ende der Zeit des Progressivismus im Sinne einer lingua franca markiert. Gleichzeitig zeigt es das Vordringen einer regressiven Rechten, die versucht, die Kapitallogik in den Territorien auf eine offenere Art und Weise voranzutreiben. 



\section{Abschließende Überlegungen: Die Dimensionen der Systemkrise}

Die Menschheit durchläuft gerade eine globale Systemkrise, eine zivilisatorische Krise (Lander und Arconda Rodríguez 2019), die verschiedene Faktoren beinhaltet und eng mit der Expansion des neoliberalen Kapitalismus und dessen Grenzen verbunden ist ${ }^{1}$. Daher schlage ich in diesem letzten Kapitel eine Reflexion über die verschiedenen Dimensionen dieser Krise, angefangen mit der sozio-ökologischen Krise und endend mit der politischen Krise in Lateinamerika, vor. Dazu greife ich den Anthropozänbegriff wieder auf, um seine Verknüpfung zur Entwicklungskritik und zum Neoextraktivismus herzustellen. Ebenso gehe ich auf einige der Konzepte ein, die die kritische Analyse der hegemonialen Entwicklungsmodelle in Europa und Lateinamerika durchziehen und die notwendig sind, um über die Alternativen zur Krise nachzudenken.

1 Die hier vertretene Sichtweise deckt sich mit der des 2017 von der Stiftung Solón (Bolivien), Attac Frankreich und Focus on the Global South herausgegebenen Sammelbandes Alternativas sistémicas [»Systemische Alternativen«], der eine Diagnose in Bezug auf die »Systemkrise« stellt und eine Reise durch verschiedene Begriffshorizonte vom Guten Leben oder vivir bien bis zu Degrowth, den Rechten der Mutter Erde, dem Ökofeminismus, den Cemeingütern und der Deglobalisierung, unternimmt. 


\section{Dimensionen der Krise: Das Anthropozän}

Der Begriff Anthropozän bezeichnet ein neues Erdzeitalter, in dem der Mensch zu einer Transformationskraft mit globaler und geologischer Tragweite geworden ist. Diese Kategorie wurde vorgeschlagen, um das Holozän, ein etwa 10.000 bis 12.000 Jahre andauerndes und durch klimatische Stabilität gekennzeichnetes Zeitalter, abzulösen, das die Expansion und Dominanz des Menschen auf der Erde ermöglichte. Die Idee des Eintritts in ein neues Erdzeitalter basiert auf der Annahme, dass wir eine gefährliche Schwelle zu abrupten und irreversiblen Veränderungen, angeführt durch die Erderwärmung und den Klimawandel sowie das massenhafte Artensterben und den daraus resultierenden Verlust von Biodiversität, um nur die Spitze des Eisbergs zu nennen, überschritten haben.

Der Begriff Anthropozän bringt zwei Wurzeln aus dem Griechi-


xaıvós (kainos), was neu oder aktuell bedeutet. Das Konzept wurde von einigen bedeutenden WissenschaftlerInnen, unter ihnen der Chemiker Paul Crutzen, im Jahr 2000 vorgeschlagen, um das Holozän $\mathrm{zu}$ ersetzen, und bezeichnet eine neue geologische Ära, in der der Mensch zu einer Transformationskraft von globaler und geologischer Reichweite wird. Die Faktoren, die als Rechtfertigungen für den Eintritt in ein neues Zeitalter sprechen, sind sehr zahlreich. Ein erstes Element bezieht sich auf den aus erhöhten Emissionen von Kohlendioxid und anderen Treibhausgasen resultierenden Klimawandel in Verbindung mit der globalen Erderwärmung. Heute ist der Anteil von Methangas und Kohlendioxid in der Atmosphäre als ein Produkt der menschlichen Emissionen um 150 \% bzw. $45 \%$ höher als noch 1750 . Infolgedessen ist die mittlere globale Temperatur seit Mitte des 20. Jahrhunderts um $0,8^{\circ} \mathrm{C}$ gestiegen, und die vom International Panel on Climate Change (IPCC) vorgesehenen Szenarien prognostizieren einen weiteren Temperaturanstieg zwischen 1,2 und $6^{\circ} \mathrm{C}$ bis zum Ende des 21. Jahrhunderts. Die WissenschaftlerInnen betrachten die Temperaturschwelle von mehr als $2^{\circ} \mathrm{C}$ als Gefahrengrenze und halten einen durchaus größeren Temperaturanstieg für möglich, wenn alles 
wie gewohnt weitergeht (business as usual). Systemorientierte Ansätze und die neuesten wissenschaftlichen Erkenntnisse zeigen, dass bereits eine geringe Abweichung der Durchschnittstemperatur der Erde unvorhersehbare und unkontrollierte Veränderungen auslösen kann.

Im Jahr 2017 stellte ein Bericht der Non-Profit-Organisation The Carbon Majors fest, dass mehr als die Hälfte der weltweiten Industrieemissionen seit 1988 von insgesamt 25 Unternehmen und staatlichen Einrichtungen stammen. Große Ölkonzerne wie ExxonMobil, Shell, $\mathrm{BP}$ und Chevron gehören $\mathrm{zu}$ den größten Emissionsverursachern. Außerdem besagt dieser Bericht, dass die Durchschnittstemperaturen bis zum Ende des Jahrhunderts um ca. $4^{\circ} \mathrm{C}$ steigen werden, wenn fossile Brennstoffe in den nächsten 28 Jahren weiterhin im derzeitigen Tempo gefördert werden.

Der zweite Alarmfaktor betrifft den Verlust der Biodiversität, die Zerstörung des Lebensgeflechts und der Ökosysteme. Dies ist ein rekursiver Prozess, da der Verlust der Biodiversität auch durch den Klimawandel beschleunigt wird. Hierbei genügt es zu sagen, dass die Rate des Artensterbens in den letzten Jahrzehnten tausendmal höher war als die normale geologische Rate. Deshalb spricht man bereits vom sechsten Massensterben, obwohl im Gegensatz zu den fünf vorhergehenden, die durch exogene Faktoren (globale Abkühlung oder im Falle des Aussterbens von Dinosauriern ein Asteroideneinschlag) erklärt werden, dieses sechste Massensterben - so die Hypothese - anthropogenen Ursprungs ist, was die Verantwortung des menschlichen Handelns und seine Auswirkungen auf das Leben auf dem Planeten in den Mittelpunkt stellt.

Bereits im Jahr 2004 hat eine Gruppe von WissenschaftlerInnen Spezies in Bezug zu einem klar definierten Gebiet gesetzt, um eine erste Risikoabschätzung der Gefährdung von Arten im Kontext des Klimawandels anhand zweier Extremszenarien vornehmen zu können. Bei einem minimalen Szenario, bei dem die globale Erwärmung auf niedrige Werte begrenzt bliebe, schätzt man, dass zwischen $22 \%$ und $31 \%$ der Arten bis 2050 vom Aussterben bedroht wären; bei einem wahrscheinlichen Maximum der globalen Erwärmung würde der Prozentsatz auf $38 \%$ bis $52 \%$ steigen. Andere Studien zeigen unterschiedliche Prozentsätze (höher oder niedriger), die Ergebnisse sind aber durchge- 
hend alarmierend. Viele Arten sind bedroht: vom einsamen Eisbären, der in einigen Jahrzehnten bei anhaltendem Schmelzen der Eisdecken des Arktischen Ozeans verschwunden sein könnte, bis hin zu den Bienen, deren Kolonien sowohl durch den Einsatz von Pestiziden als auch durch das Auftreten verschiedener Viren und natürlich auch durch den Klimawandel zusammenbrechen würden.

Nicht nur die Landökosysteme sind bedroht. So ist die Versauerung der Ozeane, das andere Gesicht der globalen Erderwärmung, ein Produkt der Kohlendioxidkonzentration, das die Chemie der Gewässer verändert und das Überleben der marinen Ökosysteme gefährdet. Seit Beginn der industriellen Revolution stieg der durchschnittliche Säuregehalt der Ozeane durch die Aufnahme von Kohlendioxid aus der Verbrennung fossiler Brennstoffe um $30 \%$. Es wird geschätzt, dass das Meer etwa eine halbe Milliarde Tonnen $\mathrm{CO}_{2}$ absorbiert hat, »was dem Gewicht von 500 Milliarden ins Meer geworfener Volkswagen Käfer entspricht«, so Bonneuil und Fressoz (2013).

In einem Text voller Ironie und scharfer Kommentare behauptet die US-amerikanische Philosophin und Feministin Donna Haraway (2016), in dem sie die Biologin Anna Tsing zitiert, dass das Holozän eine lange Periode war, in der es noch reichlich Zufluchtsgebiete gab, in denen verschiedene Organismen unter ungünstigen Bedingungen leben konnten, um dann zurückzukehren und langsam eine Strategie der Wiederbevölkerung zu entwickeln. Zwar kam es zu einem sukzessiven Aussterben, das einen erheblichen Teil der Arten aufgrund exogener Faktoren (Klimawandel und/oder große Katastrophen) verenden ließ, aber das Leben auf der Erde hat immer eine große Widerstandsfähigkeit gezeigt. Das Neuartige und auch das Drastische am Anthropozän sei, dass es die Zerstörung von Räumen und Zeiten der Zuflucht für jeden Organismus, seien es Tiere, Pflanzen oder Menschen, mit sich bringt, und das nicht nur wegen des Ausmaßes, sondern auch wegen der Geschwindigkeit des Prozesses. Alles deutet darauf hin, dass die Beschleunigung der Veränderungen auch die Anpassungsmöglichkeiten erschweren wird. Folglich ist das Anthropozän weniger ein neues Zeitalter als ein »Wendepunkt«, der uns zu der Erkenntnis zwingt, dass »das, was kommt, nicht wie das sein wird, was vorher war.« 
Ein weiterer kritischer Faktor betrifft die Veränderungen der biogeochemischen Kreisläufe, die für die Aufrechterhaltung des Gleichgewichts von Ökosystemen von grundlegender Bedeutung sind. Wie beim Kohlenstoffkreislauf wurden die für die Reproduktion des Lebens wichtigen Kreisläufe von Wasser, Stickstoff, Sauerstoff und Phosphor in den letzten beiden Jahrhunderten von Menschen verändert. Die übermäßige Zunahme der industriellen Aktivitäten, die Abholzung der Wälder, die Verschmutzung der Böden und Gewässer durch die Einwirkung von Düngemitteln führen zu einer Veränderung dieser Lebenszyklen. Zum Beispiel führte der wachsende Energiebedarf zu einer Veränderung des Wasserkreislaufs durch den Bau von Staudämmen. »Wir haben die Hälfte der Flüsse der Welt gestaut, mit der beispiellosen Geschwindigkeit von einem Damm pro Stunde, und in bisher nicht gekannten Dimensionen von mehr als 45 Tausend Dämmen« in mehr als 140 Ländern der Welt, mit einer Höhe von mehr als vier Stockwerken, laut der Website der World Commission on Dams (Castro 2009). Dies führt zur Zwangsumsiedlung von Millionen von Menschen. Neben den Auswirkungen auf die Ökosysteme, dem Verlust von Naturressourcen und dem für immer unter Wasser stehenden Kulturerbe haben die Staudämme weltweit zwischen 40 und 80 Millionen Menschen vertrieben, obwohl einige betonen, dass diese Zahl eher konservativ ist und durchaus von 100 Millionen Vertriebenen ausgegangen werden kann. Die meisten davon sind indigene und bäuerliche Bevölkerungsgruppen. Die beiden bevölkerungsreichsten Länder der Welt, China und Indien, haben die größte Zahl an Vertriebenen; in unserer Region führt Brasilien das Ranking mit mehr als einer Million Vertriebenen an.

Hinzu kommt die Zunahme der Weltbevölkerung, denn die Anzahl der Menschen stieg von 900 Millionen im Jahr 1800 auf fast 7.5 Milliarden im Jahr 2018. Der globale ökologische Fußabdruck großer Teile der Menschheit übersteigt heute die Regenerationsfähigkeit der Ökosysteme; er ist allein zwischen 1970 und 1997 um 50 \% gestiegen. Heute verbrauchen wir eineinhalb Mal so viel, wie die Erde auf nachhaltige Weise bereitstellen kann. Das bedeutet, dass die Erde mehr als anderthalb Jahre brauchen wird, um das zu regenerieren, was wir in einem Jahr verbraucht und an Abfall produziert haben. Die Realität dieses Res- 
sourcenverbrauchs ist bereits unhaltbar und wird sich noch weiter zuspitzen, denn es wird erwartet, dass die Weltbevölkerung bis 2050 auf 10 Milliarden Menschen angewachsen sein wird.Die meisten von ihnen werden in Schwellen- oder Entwicklungsländern leben. Wenn das derzeitige Konsumverhalten anhält, wird geschätzt, dass wir bis 2030 das Äquivalent von zwei Planeten Erde benötigen, um die Menschheit zu versorgen.

Ein weiterer alarmierender Faktor ist die Veränderung des Konsummodells, das auf dem Schema der geplanten Obsoleszenz basiert, das die Lebensdauer der Produkte begrenzt und die Menschen zwingt, sie immer wieder zu erneuern, und zwar in Abhängigkeit von der Maximierung des Kapitalnutzens. Eine sozial-ökologisch unhaltbare Praxis, die schon früh von den Automobilherstellern initiiert wurde und die seit den 1960er Jahren durch den industriellen Sektor, der von der Produktion von Haushaltsgeräten, Computern, Mobiltelefonen bis hin zu Textilien reicht, noch verschärft wurde. Dieser Prozess ist wiederum Teil einer viel umfangreicheren Entwicklung, die mit den Mutationen im Ernährungsmodell der letzten Jahrzehnte zusammenhängt. Wir haben, gefördert durch staatliche Politik, Marketinglogiken und mächtige Wirtschaftslobbies, die hinter dem Rücken der Zivilgesellschaft agieren, einen deutlichen Wandel hin zu einem Ernährungsmodell erlebt, das enorme Auswirkungen auf unsere Gesundheit, auf das Leben von Tieren, Pflanzen und Feldern hat. Es handelt sich um ein Modell, das von den großen Agrar- und Nahrungsmittelkonzernen der Welt aufgebaut wurde und mit der Degradierung aller Ökosysteme einhergeht: Ausbreitung von Monokulturen (wie Soja und Ölpalmen), die zur Vernichtung der Biodiversität führen, sowie eine Tendenz zur Überfischung, die Verschmutzung durch Düngemittel und Pestizide, die Abholzung und das Land Grabbing. All diese Produktionsformen und die Schädigung der Ökosysteme sind für den Anstieg der Treibhausgasemissionen verantwortlich, nicht nur während des Produktionsprozesses, sondern auch beim Transport der Waren. 


\section{Anthropozän, Kritik des Neo-Extraktivismus und Alternativen}

Das Konzept des Anthropozän hat eine enorme Wirkung entfaltet. Schon bald wurde es nicht mehr nur auf dem Gebiet der sogenannten Geowissenschaften diskutiert, sondern auch in den Sozial- und Geisteswissenschaften, einschließlich des künstlerischen Bereichs, weshalb es zu einem gemeinsamen Kontaktpunkt für GeologInnen, ÖkologInnen, KlimatologInnen, HistorikerInnen, PhilosophInnen, KünstlerInnen und KunstkritikerInnen und anderen werden konnte. Für eine Vielzahl von WissenschaftlerInnen, darunter Paul Crutzen, stellt die Industrielle Revolution, das heißt die Erfindung der Dampfmaschine und der Beginn der Förderung von fossilen Brennstoffen - zuerst Kohle, dann Erdöl - den Eintritt in eine neue Ära dar. Auf diese erste folgte eine zweite, »die große Beschleunigung genannte Phase, die nach 1945 begann und durch eine Vielzahl von Indikatoren für menschliche Aktivitäten veranschaulicht wurde, die von der $\mathrm{zu}$ nehmenden Petrolisierung der Gesellschaften, der atmosphärischen Konzentration von Kohlenstoff und Methan bis hin $\mathrm{zu}$ steigenden Zahlen von Staudämmen, den Veränderungen des Stickstoff- und Phosphorkreislaufs und dem drastischen Verlust der Biodiversität reichen. All diese Indikatoren zeigen eine exponentielle Zunahme der Auswirkungen menschlicher Aktivitäten auf den Planeten seit 1950.

Für andere, wie die Anthropocene Working Group, bestehend aus einer Gruppe von WissenschaftlerInnen der University of Leicester und der British Geological Survey unter der Leitung von Jan Zalaslewicz ist der Planet zu einem späteren Zeitpunkt in eine neue geologische Ära, also das Anthropozän, eingetreten. Nach sieben Jahren Arbeit, Ende 2016, führte die Gruppe von GeologInnen stratigraphische Tests durch, die das Vorhandensein von Aluminium, Beton, Plastik, Überresten von Atomtests, erhöhtem Kohlendioxid, radioaktivem Fallout und anderen Spuren in Sedimenten zeigten. Folglich stimmte diese Gruppe für die These, dass das Anthropozän 1950 mit den radioaktiven Abfällen von Atombomben begonnen hätte. Die Markierung für diese Veränderung stellen die radioaktiven Abfälle von Plutonium dar, die Mitte des 20. 
Jahrhunderts durch die zahlreichen Atombombentests freigesetzt wurden.

Für andere AnalytikerInnen wie den marxistischen Historiker Jason Moore stellt der Übergang zu einem neuen Erdzeitalter dagegen einen längeren Prozess dar, für dessen Analyse die Ursprünge des Kapitalismus und die Ausdehnung der Warengrenzen im langen Mittelalter in den Blick zu nehmen wären. In diesem Sinne plädiert Moore auch dafür, dass neue Zeitalter »Kapitalozän« zu nennen. Die Kapitalzyklen hätten ein historisch-geographisches Modell etabliert, das auf einer raschen Aneignung sowie - sobald die Ressourcen erschöpft waren - einer geographischen Ausdehnung und Diversifizierung beruhte: »Ist das Land verbraucht? Wir ziehen zur Grenze. So lautete der Wahlspruch des Frühkapitalismus« (Moore 2013). Die aktuelle Krise muss also als ein langwieriger Prozess gelesen werden, in dem neue Ordnungsformen der Beziehung zwischen Mensch und der übrigen Natur entstehen.

Aus meiner Perspektive ist es notwendig, die kritische Reichweite dieses Konzepts zu fördern, das Anthropozän im Sinne einer Ausweitung der Merkantilisierung und der Grenze zu denken, was uns dazu zwingt, auf die Kritik am neoliberalen Kapitalismus zurückzukommen. Dies bedeutet jedoch nicht, dass der Gedanke des gesamten Anthropozäns aufgegeben werden sollte. Vielmehr gilt es, die Spannung hervorzuheben, die ihn durchzieht, denn es handelt sich um einen umstrittenen Begriff, der von verschiedenen Narrativen durchzogen ist, die nicht alle übereinstimmen, und zwar nicht nur in Bezug auf den Beginn des neuen Zeitalters, sondern vor allem in Bezug auf die möglichen Auswege aus der systemischen Krise. ${ }^{2}$

2 In einem Szenario, in dem keine der beteiligten Mächte den ersten Schritt machen will, bereitet der Kapitalismus angesichts der immer knapper werdenden Claubwürdigkeit globaler Vereinbarungen zur Kontrolle der $\mathrm{CO}_{2}$-Emissionen seinen Plan B vor, um das Projekt der kapitalistischen Moderne zu recyceln, ohne den Kapitalismus verlassen zu müssen. Dieser Plan B wird als GeoEngineering bezeichnet und basiert auf dem Prinzip, dass es möglich ist, die Risiken der globalen Erderwärmung zu überwinden, indem bewusst in das Klima auf globaler Ebene eingegriffen wird. Zum Thema siehe Hamilton (2013). 
Das Anthropozän als kritische Diagnose fordert uns heraus, das sozio-ökologische Problem von einem anderen Standpunkt aus zu betrachten. Es etabliert die Idee, dass die Menschheit eine Grenze verschoben hat, die uns mit zunehmend unvorhersehbaren, nicht-linearen und groß angelegten Reaktionen der Natur konfrontiert. Dabei wird klar, dass es sich nicht nur um eine Krise der Menschheit, des Anthropos im allgemeinen Sinne, handelt. In dem Maße, in dem die dominanten wirtschaftlichen und politischen Akteure weiterhin nicht nachhaltige Entwicklungsmodelle fördern, ist nicht nur das menschliche Leben, sondern auch das anderer Arten und das des Erdsystems insgesamt, zumindest wie wir es kennen, gefährdet. Als kritische Diagnose bringt das Anthropozän folglich die Infragestellung der aktuellen Entwicklungslogiken mit sich.

In dieser Hinsicht kommt in der globalisierten Peripherie und durch den Neo-Extraktivismus die Kommodifizierung aller Produktionsfaktoren voll zum Ausdruck. Dies hängt mit der gegenwärtigen Phase des neoliberalen Kapitalismus zusammen, der die Ausweitung der Grenzen der Kapitalausbeutung durch die Auferlegung nicht nachhaltiger Entwicklungsmodelle in großem Maßstab zur Folge hat. Auf diese Weise werden außerordentliche Rentabilität, die Zerstörung von Territorien und die Enteignung ganzer Bevölkerungsgruppen miteinander kombiniert. Hinzu kommt eine Zunahme von Extremereignissen: Brände, Überschwemmungen, Dürren, die nicht nur weit verbreitete Phänomene auf dem Planeten sind, sondern auch mit der Politik verschiedener Regierungen verbunden sind, die durch Maßnahmen zugunsten der Agrarindustrie und ihrer Ernährungsmodelle, des Großbergbaus, der Expansion der Erdölgrenze, der Großstaudammprojekte etc. gefördert werden. Insgesamt ist der $\mathrm{Zu}$ sammenhang zwischen Anthropozän, Ausweitung der Warengrenze und Verschärfung des Neo-Extraktivismus aus Sicht des Globalen Südens unbestreitbar.

Ausgehend vom Globalen Süden hat dies zu einer Debatte nicht nur über die bereits unübersehbaren Folgen des Neo-Extraktivismus geführt, sondern auch über den möglichen Umgang mit der systemischen Krise. Die Annahme der sozio-ökologischen und zivilisatorischen Krise 
des Anthropozäns impliziert die Herausforderung, über Alternativen zum dominanten Extraktivismus nachzudenken und Übergangsstrategien auszuarbeiten, die den Weg $\mathrm{zu}$ einer post-extraktivistischen Gesellschaft bereiten. Um dies $\mathrm{zu}$ erreichen, ist es notwendig, jene hegemonialen Visionen zu überwinden, die die Entwicklung weiterhin aus einer produktivistischen Perspektive (unbestimmtes Wachstum) betrachten, als ob die Naturgüter unerschöpflich wären, während sie den Menschen als autonom und als jemanden außerhalb der Natur oder über ihr stehend begreifen. Zudem erfordert es ein Nachdenken über den Übergang und den Ausstieg aus dem aktuellen Entwicklungsmuster, das nicht nur den dominanten Neo-Extraktivismus - im Sinne des Aneignungsmodus der Natur und des Akkumulationsmodells -, sondern auch die vorherrschenden Zirkulations- und Konsummuster einschließt, um umfassende und systemische Alternativen $\mathrm{zu}$ entwickeln.

Kurz gesagt, das Anthropozän erfordert als kritische Diagnose ein Überdenken der Krise aus einer systemischen Perspektive. Die Umweltfrage kann nicht auf einen weiteren Kostenpunkt in der Buchhaltung eines Unternehmens im Namen seiner vermeintlichen sozialen Verantwortung reduziert werden. Sie kann auch nicht auf eine Politik der ökologischen Modernisierung oder einer Green Economy reduziert werden, die grob auf die Kontinuität des Kapitalismus durch die $\mathrm{Zu}$ sammenführung von Marktlogik und der Verteidigung neuer, als »sauber « proklamierter Technologien verweist. Schließlich kann die aktuelle sozio-ökologische Krise nicht nur als ein weiterer Aspekt oder eine weitere Dimension der öffentlichen Agenda oder gar als eine weitere Dimension der sozialen Konflikte gesehen werden. Sie muss unbedingt aus einer holistischen, integrativen und betont inter- und transdisziplinären Perspektive heraus gedacht werden.

Aus theoretischer Sicht und übereinstimmend mit den Ansätzen von Alberto Acosta und Ulrich Brand (2017) ist es möglich, über den Übergangsprozess nachzudenken, indem man zwei Konzepte artikuliert, die auf globaler Ebene zunehmend im Protestfeld verwurzelt sind: Post-Extraktivismus und Postwachstum. Hierbei handelt es sich um zwei mehrdimensionale Horizontkonzepte, die gemeinsame Merkma- 
le oder kritische Elemente aufweisen: So liefern sie beispielsweise eine kritische Diagnose des gegenwärtigen Kapitalismus, nicht nur im Hinblick auf die Wirtschafts- und Kulturkrise, sondern auch aus einem globaleren Ansatz heraus, verstanden als eine sozio-ökologische Krise zivilisatorischen Ausmaßes. Beide betonen die ökologischen Grenzen des Planeten und kritisieren gleichzeitig die Nichtnachhaltigkeit der weltweit verbreiteten Konsum- und Ernährungsmodelle, sowohl im Norden als auch im Süden. Beides sind schließlich Begriffe, die den Ausgangspunkt für ein Nachdenken über Veränderungshorizonte und zivilisatorische Alternativen bilden, die auf einer anderen Umweltrationalität basieren, die sich von der rein ökonomischen unterscheidet, welche den Prozess der Kommodifizierung des Lebens in seinen verschiedenen Aspekten vorantreibt.

In der Tat wird der Übergangsprozess von Lateinamerika aus anhand neuer Formen der territorialen Nutzung gedacht, von denen einige am Anfang stehen, andere bereits in Kraft sind und die im $\mathrm{Zu}$ ge der sozialen Konflikte und Widerstände, die einen antikapitalistischen Charakter annehmen, entstanden sind. Diese neuen Lebensformen werden von einem politisch-ökologischen Narrativ begleitet, das unter anderem mit Konzepten wie dem Buen Vivir, den Naturrechten, den Gemeinschaftsgütern, dem Post-Development und der Ethik der Sorge verbunden ist. All diese Konzepte basieren auf dem Schutz des Gemeinsamen, das heute als einer der Schlüssel auf der Suche nach einem neuen emanzipatorischen Paradigma erscheint. Sie beruhen auf der antagonistischen Grammatik der sozialen Bewegungen, und zwar sowohl in den zentralen Ländern, wo der Kampf zum Schutz des Gemeinsamen heute gegen die Anpassungs- und Privatisierungspolitik (Neoliberalismus) und gegen die Expansion extremer Energien definiert wird, als auch in den peripheren Ländern, in denen sich der Kampf vor allem gegen die verschiedenen und vielfältigen Formen des entwicklungspolitischen Neo-Extraktivismus richtet.

Gewiss ist es zur Umkehrung der Logik eines unbegrenzten Wachstums notwendig, andere Formen der sozialen Organisation zu erkunden und voranzutreiben, die auf Gegenseitigkeit und Umverteilung basieren und die der Marktlogik klare Grenzen setzen. In Lateiname- 
rika und den anderen Ländern des Südens gibt es zahlreiche Konzepte aus der Sozial- und Solidarwirtschaft, deren soziale Bezugspunkte die am meisten ausgegrenzten Gruppen miteinbeziehen (Frauen, Indigene, Jugendliche, ArbeiterInnen, KleinbäuerInnen) und deren Sinn menschlicher Arbeit darin besteht, Nutzwerte oder Lebensmittel zu produzieren. So entsteht eine Vielfalt an Perspektiven zur Selbstorganisation und des Selbstmanagements von Produktionsprozessen. Es kommt nicht $\mathrm{zu}$ entfremdeten oder unpersönlichen Arbeitsformen, sondern zu solchen, die mit der Reproduktion des Soziallebens und der Schaffung neuer Gemeinschaftsformen verbunden sind. Zum Beispiel wurden in Argentinien, einem Land, das so sehr vom Sojaanbau geprägt ist, in Abgrenzung zum dominanten Agrarmodell Netzwerke durch Gemeinden geschaffen, um eine agrarökologische Landwirtschaft zu fördern, die gesunde Lebensmittel ohne den Einsatz von Agrotoxinen, mit geringen Kosten und geringerer Rentabilität und dem Einsatz von mehr Arbeitskräften produziert. Es entsteht ein neuer Kontext der Agrarökologie mit einer Vielfalt an Erfahrungen, die versuchen, sich am Rande des dominanten Sojamodells $\mathrm{zu}$ vernetzen. Das heute dominierende Modell des Sojaanbaus basiert auf dem Export genveränderter Pflanzen. Obwohl die alternativen Ansätze bescheiden, lokal begrenzt und stets von Unsicherheiten und einer möglichen Kooptation bedroht sind, hinterlassen diese Erfahrungen der Selbstorganisation ihre Spuren durch die Schaffung eines neuen sozialen Gefüges und einer Reihe von Möglichkeiten und Perspektiven, die es zu erforschen und $\mathrm{zu}$ fördern gilt.

Auf der anderen Seite vermischen sich in Europa die vielfältigen Dimensionen der Krise mit dem Hinterfragen und dem Scheitern des Neoliberalismus. Dies wird zum einen sichtbar in der verstärkten Ausgrenzung großer Bevölkerungssektoren, die nicht mehr in die immer größere Ungleichheiten produzierende kapitalistische Globalisierung eingebunden werden. Zum anderen tritt die Etablierung einer konsumorientierten Lebensweise hervor, die den sozialen Metabolismus des Kapitals (und damit die Nachfrage nach Rohstoffen und Energie) rasend schnell vorantreibt. Im Zusammenhang einer Krise nicht nur politischer und wirtschaftlicher, sondern auch kultureller Art tauchte ab 2008 
die in den 7oer Jahren entstandene Wachstumskritik wieder auf, die seither wiederauflebt. Weit entfernt von der wörtlichen Bedeutung, mit dem manche den Begriff »Degrowth « (als einfaches Gegenstück zum Wirtschaftswachstum) assoziieren, vertieft die in den letzten Jahrzehnten in Europa entwickelte Sammlung an Erfahrungen die Diagnose der Systemkrise (die sozialen, wirtschaftlichen und ökologischen Grenzen des Wachstums, verbunden mit dem aktuellen kapitalistischen Modell) und eröffnet eine Vision der Dekolonialisierung hin zu einer neuen sozialen und politischen Sprache, in der verschiedene Vorschläge und Alternativen hervorstechen: Beispiele sind etwa Schuldenerlass, ziviler Ungehorsam, ökologische Gemeinschaften, Urban Gardening, die »Indignados« in Spanien, Aufteilung der Erwerbsarbeit, bedingungsloses Grundeinkommen. Zum Beispiel werden im Rahmen der Energiewende so genannte Transition Towns, also Übergangsstädte gefördert. Es handelt sich um eine pragmatische Bewegung zugunsten der Agrarökologie, der Permakultur, des Konsums von lokal und/oder kollektiv produzierten Gütern, von Degrowth und zur Wiedererlangung der Fähigkeiten für ein Leben in und im Einklang mit der Natur. Die TransitionTown-Bewegung, die 2006 in Irland entstanden ist, hat sich zum Ziel gesetzt, schlicht lebende Gesellschaften zu schaffen, die saubere und erneuerbare Energien nutzen, um dabei einen starken Anstieg der Energieeffizienz zu erreichen. Diese Übergangsgemeinschaften versuchen, soziale Resilienz gegen den fortschreitenden sozialen Zusammenbruch durch den Klimawandel, die Erschöpfung fossiler Brennstoffe und den Niedergang der politischen Ordnung aufzubauen.

\section{Gaia und die Wege der Interdependenz}

Die Abkehr vom Anthropozentrismus hat tiefe philosophische, ethische und politische Auswirkungen. Sie zwingt uns, uns selbst als anthropos zu überdenken, aber sie drängt uns auch, die Verbindung zwischen Gesellschaft und Natur, zwischen Mensch und Nichtmensch neu zu überdenken. Es trifft zwar zu, dass wir vor Jahrhunderten die holistische Vision der Natur, Gaia, Gea oder Pachamama, wie sie unsere Vorfahren 
uns erklärten, aufgegeben haben. Als Kinder der Moderne oder als von ihr kolonisierte Nachkommen haben wir uns aber mit der Natur auf Grundlage anthropozentrischer oder androzentrischer Episteme verbunden, deren Beharrlichkeit und Erneuerung - weit davon entfernt, uns zu einer Lösung der Krise zu führen - schließlich zu einem zentralen Teil des Problems geworden sind.

In ihrer kritischsten Ausrichtung bedeutet die Wende des Anthropozäns eine Infragestellung des kulturellen Paradigmas der Moderne, das auf einer instrumentellen, für die Logik der Kapitalexpansion funktionalen Vision der Natur basiert. Dahingehend erinnern uns die Anthropologie und die kritische Philosophie der letzten Jahrzehnte eindringlich an die Möglichkeit anderer Methoden der Konstruktion von Verbindungen mit der Natur, zwischen dem Menschlichen und dem Nichtmenschlichen. Mit anderen Worten entwickelten nicht alle Kulturen und nicht alle Zeitalter, auch nicht die des Westens, einen dualistischen Zugang zur Natur. Nicht alle Völker gingen den gleichen Weg und haben die Natur isoliert oder sie als eine nebensächliche äußere Umgebung, die dem Menschen untergeben ist, betrachtet. Es existieren andere Ansätze, zum Beispiel relationale oder generative, die auf einer dynamischeren Vision basieren, wie es in einigen Kulturen des Orients der Fall ist. Dort ist das Konzept der Bewegung und des Werdens das Prinzip, das die Welt regiert und das in der Natur verkörpert wird. Ein anderes Beispiel sind jene an die Immanenzphilosophie angelehnten Visionen der Ureinwohner Amerikas, die den Menschen als Teil der Natur begreifen und nicht als separates Element.

Diese relationalen Ansätze, die die Interdependenz alles Lebendigen unterstreichen, berücksichtigen andere Formen der Beziehung zwischen Lebewesen, zwischen Mensch und Nichtmensch und können verschiedene Bezeichnungen haben: Animismus nach dem Anthropologen Philippe Descola; amazonischer oder amerindischer Perspektivismus nach Eduardo Viveiros de Castro. Für Descola (2011) gilt der Naturalismus (Dualismus von Gesellschaft - Natur), der mit der westlichen Kultur verbunden ist und auf der Idee basiert, dass der Mensch dieselbe physische Realität, also die Körperlichkeit, mit dem Tier teilt. Demnach unterscheiden sich beide durch ihre Innerlichkeit. 
Im Animismus haben alle Wesen ein ähnliches Inneres, aber unterscheiden sich durch ihre Körper. Der amerindische Perspektivismus besagt, dass die Welt von vielen Arten oder Wesen bevölkert ist, die mit Bewusstsein und Kultur ausgestattet sind, und dass jedes dieser Wesen sich selbst als menschlich sieht und die anderen als nichtmenschlich, d.h. als Tiere oder Geister betrachtet. Im Gegensatz zur modernen Sichtweise ist die Gemeinsamkeit zwischen Menschen und Nichtmenschen nicht das Animalische, sondern das Menschliche. Das Menschliche wird nicht zur Ausnahme, sondern ist die Regel; jede Spezies versteht sich als menschlich, also als Subjekt, unter der Spezies Kultur: "Die Menschheit ist der universelle Hintergrund des Kosmos. Alles ist menschlich« (Viveiros de Castro 2008).

Diese Formen der Beziehung zu und der Aneignung von Natur stellen die Dualismen, die die Moderne ausmachen, in Frage. Dahingehend hält der Kolumbianer Arturo Escobar Folgendes fest:

»Anthropologen, Geographen und Umweltaktivisten haben zunehmend gezeigt, dass viele ländliche Cemeinschaften in der Dritten Welt die Natur auf eine Weise »konstruieren «, die sich auffallend von den vorherrschenden modernen Formen unterscheidet: Sie bezeichnen und nutzen daher die natürliche Umwelt auf ganz besondere Art und Weise. Ethnographische Studien über die Szenarien in der Dritten Welt zeigen eine Reihe signifikant unterschiedlicher Praktiken des Denkens, der Beziehung, der Konstruktion und des Erlebens des Biologischen und des Natürlichen auf« (Escobar 2000, 71).

Diese »relationalen Ontologien«, wie Escobar (2011 und 2014) sie in Anlehnung an den Anthropologen Mario Blaser nennt, sehen das Territorium und seine ortsbezogenen Logiken als Voraussetzungen für das Mögliche. Die gegenseitige Beziehung erzeugt Synergieeffekte zwischen der Welt der Männer und Frauen mit anderen Welten, die die Lebenswelt der Menschen umgeben. Diese Räume materialisieren sich in Praktiken oder in Bergen und Seen, obwohl angenommen wird, dass sie Leben führen oder belebte Räume sind, was natürlich aus der Perspektive des europäischen Positivismus schwer zu definieren ist. 
Wenn es andererseits darum geht, unsere Beziehung zur Natur aus einer relationalen Perspektive zu überdenken, eröffnen die Care-Ethik und die Ethik des Ökofeminismus zweifellos andere mögliche Wege. Ihre Perspektiven können uns helfen, die Verbindungen zwischen dem Menschlichen und dem Nichtmenschlichen neu zu beleuchten und die auf der Idee der Autonomie und des Individualismus basierende reduktionistische Vision zu hinterfragen. Sicherlich bietet die Care-Ethik einen weiteren Zugang bei der notwendigen Aufgabe, unsere Verbindung zur Natur neu zu überdenken, indem sie den Begriff der Interdependenz in den Mittelpunkt stellt, der im Schlüssel der zivilisatorischen Krise als Ko-abhängigkeit zu verstehen ist. Die universale Ausweitung der Care-Ethik, wie Carol Gilligan (2015) feststellt, eröffnet einen Prozess größerer Befreiung, nicht nur im feministischen Zusammenhang, sondern für die gesamte Menschheit.

Diese Handlungslinie spiegelt sich in der wachsenden Beteiligung von Frauen im Kampf gegen den Neo-Extraktivismus und seinen verschiedenen Erscheinungsbildern wider. Diese Kämpfe machen den Weg frei für eine Dynamik, die die von der westlichen Moderne konsolidierte dualistische Vision in Frage stellt, welche die Natur als etwas Außenstehendes betrachtet, das beherrscht und ausgebeutet werden kann. An diesem entscheidenden Punkt stellt der populare Feminismus ein anderes Verhältnis her zwischen Gesellschaft und Natur durch die Bekräftigung des Begriffs der Interdependenz, in dem der Mensch als Teil der Natur verstanden wird. Andererseits bringt dieser prozessuale Charakter der feministischen Kämpfe auch eine Hinterfragung des Patriarchats als Modell der Herrschaft eines Geschlechts über ein anderes mit sich, das auf einem binären und hierarchischen Muster basiert, das das Männliche vom Weiblichen trennt und privilegiert.

Die führende Rolle, die die Frauen in Lateinamerika im Kampf gegen die Ausdehnung der Rohstoffgrenze und die Landnahme übernommen haben, ist ein paradigmatisches Beispiel für diesen zweiseitigen Prozess. Es handelt sich um persönliche und zugleich kollektive Stimmen, die uns, wenn wir ihnen aufmerksam zuhören, verschiedene Ebenen des Denkens und Handelns erkennen lassen. Denn hinter der Anprangerung und dem Bezeugen der Geschehnisse kann man nicht nur 
den konkreten und verteidigenden Kampf der Frauen in den Territorien ausmachen, der eine starke Identifikation mit ihren Gebieten und deren zentralen Reproduktionszyklen beinhaltet, sondern auch die Entmystifizierung des Entwicklungsmythos und den Aufbau einer alternativen Beziehung zur Natur. Nicht selten wird die Forderung nach einer freien, ehrlichen Stimme, einer »eigenen « Stimme sichtbar. Diese stellt das Patriarchat in all seinen Dimensionen in Frage und versucht, die Fürsorge an einen zentralen und befreienden Ort zu verlagern, der in unbestreitbarer Weise mit unserer menschlichen Existenz verbunden ist. So wird in einer zunehmend durch die Kommodifizierung geprägten Welt, in der die Gesamtheit unserer natürlichen Gemeingüter zunehmend dem Druck des neoliberalen Kapitalismus ausgesetzt ist, die Care-Ethik zu einem Fundament für ein Überdenken der Geschlechterbeziehungen sowie der Beziehungen zur Natur.

Auf diese Weise haben sich im Laufe der Kämpfe Narrative herausgebildete, die Territorialitäten anders bewerten sowie andere Wege der Verbindung mit der Natur und der Repräsentation der Mutter Erde suchen. Diese erschaffen ein neues relationales Paradigma, das auf Reziprozität, Komplementarität und Fürsorge basiert. Diese Aspekte verweisen auf alternative Möglichkeiten der Aneignung und des Austauschs von Wissen sowie auf andere Formen der sozialen Organisation. Diese neuen Artikulationsweisen werden von verschiedenen politisch-ideologischen Matrizen unterstützt, darunter antikapitalistische, ökologische, indianistische, feministische und anti-patriarchalische Perspektiven, die aus der heterogenen Welt der Subalternen stammen und das Feld der Geistes- und Sozialwissenschaften, das der Geowissenschaften, und sogar das der Künste, verbunden mit der ästhetischen Avantgarde, miteinbeziehen. Diese von unten konstruierten Artikulationsweisen bilden die unausweichlichen Ausgangspunkte im Aufbau eines neuen Zusammenlebens und anderer Wege, diese Erde zu bewohnen. Insgesamt nehmen die relationalen Ansätze unter den Bedingungen der systemischen Krise eine neue Bedeutung an: Sie zeigen uns, dass wir in einer Welt leben, in der die ontologische Pluralität auf einer Vielfalt der Welten, der »Pluriversen«, beruht, wie Escobar her- 
vorhebt. Dies hat zur Folge, dass wir andere Arten des Verständnisses von Kultur und der Lebensgestaltung respektieren.

\section{Die Dimensionen der Krise in Lateinamerika}

Bis vor einigen Jahren nahm man an, dass sich Lateinamerika anders als der Rest der Welt im Prozess befand, die soziale Ungleichheit zu reduzieren, anstatt sie zu erhöhen. Gegen Ende des Rohstoffbooms zeigen die sozialen und wirtschaftlichen Indikatoren nach mehr als zehn Jahren des Wachstums und der Ausweitung des Konsums jedoch ein besorgniserregendes Bild. Die lateinamerikanischen Regierungen, insbesondere die progressiven, haben die Sozialausgaben erhöht, die Armut durch sozialpolitische Maßnahmen verringert und die Situation der einkommensschwächsten Teile der Bevölkerung durch Lohnerhöhungen und das Anheben des Konsumniveaus verbessert. Dennoch haben sie die Ungleichheit nicht verringert. Dadurch, dass - wie im vorigen Kapitel erläutert - nichts gegen die Interessen der mächtigsten Sektoren unternommen wurde, indem keine progressiven Steuerreformen durchgeführt wurden, bestehen die Ungleichheiten fort und es kam zu einer weiteren wirtschaftlichen Konzentration und Landnahme.

Auflängere Sicht entfaltete die Ausbreitung des Neo-Extraktivismus eine Reihe von Nachteilen, welche die - ehemals als These verteidigten - komparativen Vorteile aus den Boomjahren des Rohstoffkonsenses zunichte machten. Einerseits führte der Neo-Extraktivismus nicht zu einem Aufstieg in der produktiven Matrix, sondern wieder zu einer stärkeren Ausrichtung der lateinamerikanischen Volkswirtschaften auf den Primärsektor. Dies wurde durch den Eintritt Chinas, einer Macht, die sich schnell als ungleicher Partner in der gesamten lateinamerikanischen Region herausstellte, noch verschärft. Gleichzeitig führte der zunehmende Rückgang der Rohstoffpreise zu einem Defizit in der Handelsbilanz, was die Regierungen dazu veranlasste, mehr Schulden zu machen und die Zahl der Förderprojekte zu erhöhen. Hierdurch gerieten sie in eine absurde Spirale, die zur Konsolidierung 
eines abhängigen Primärexportmusters führt und den Hergang der Menschenrechtsverletzungen noch verschärft.

Darüber hinaus ist der Zusammenhang zwischen Neo-Extraktivismus, Landnahme und Ungleichheit offensichtlich. Lateinamerika ist nicht nur die am meisten von Ungleichheit geprägte Region der Welt, sondern auch die Region mit der schlechtesten Landverteilung weltweit. Dies ist auf das Vordringen von Monokulturen und auf Enteignungen zugunsten von Großunternehmen und privaten Landbesitzern zurückzuführen. In dieser Hinsicht hat der Neo-Extraktivismus mit seinen Monokulturen tiefe Auswirkungen auf die ländliche Umwelt, was dazu führte, dass der Streit um Land zum Nachteil armer und benachteiligter Bevölkerungsgruppen neu definiert wurde. So wurde die Erweiterung der landwirtschaftlichen Nutzfläche zugunsten der großen Wirtschaftsakteure vorgenommen, die an der Einführung genveränderter Pflanzen etwa bei Sojabohnen, Ölpalmen, Zuckerrohr etc. interessiert sind. Die Daten der Agrarstatistiken aus 15 Ländern zeigen, dass »insgesamt in der Region $1 \%$ der größten landwirtschaftlichen Betriebe mehr als die Hälfte der landwirtschaftlichen Fläche einnimmt. Mit anderen Worten: $1 \%$ der Betriebe nehmen mehr Land in Anspruch als die restlichen $99 \%$ (Oxfam 2016).

Abgesehen von den internen Unterschieden weisen die vorherrschenden Entwicklungsmodelle eine gemeinsame Denkweise auf: großer Umfang, intensive Ausbeutung des Gebiets, verstärkte Auswirkungen auf die Umwelt sowie auf die soziale und gesundheitliche Situation der dort lebenden Menschen, Vorrang großer Unternehmen, eingeschränkte Demokratie und Verletzung von Menschenrechten. In diesem Sinne sei auch daran erinnert, dass Lateinamerika ein weiteres trauriges Ranking anführt: Es ist die Region, in der weltweit die meisten MenschenrechtsverteidigerInnen und UmweltaktivistInnen ermordet werden. Dies sind düstere Anhaltspunkte, die sich im letzten Jahrzehnt mit der Ausweitung der extraktivistischen Grenze und der Kriminalisierung von sozio-ökologischen Protesten verschärft haben. In diesem Sinne macht der Beginn einer neuen Aufeinanderfolge von Menschenrechtsverletzungen die Grenzen der derzeit in der Region umgesetzten Modelle demokratischer Regierungsführung und noch 
mehr der Rückzug der Grenzen der Rechte deutlich. Zu diesen gehören sowohl die Verletzung grundlegender politischer Rechte, darunter das Recht auf die Teilnahme an kollektiven Entscheidungen (z.B. Konsultationen, Referenden) als auch die Verletzung von territorialen und ökologischen Rechten, die in den neuen Verfassungen sowie in der nationalen und internationalen Gesetzgebung enthalten sind.

Diese unbestreitbare Realität, die die Demokratie beerdigt und das soziale Gefüge in Folge des hegemonialen Neo-Extraktivismus negativ umgestaltet, hat neue Barrieren zwischen den verschiedenen widerständigen Narrativen auf dem Kontinent errichtet. Dies gilt auf der einen Seite ganz besonders für die progressistischen Populisten und die entwicklungsorientierten Progressiven mit ihrer Berufung auf den Staat und ihrer Tendenz zur Konzentration und Personalisierung von Macht. Auf der anderen Seite basiert diese Entwicklung auf einer radikalen politischen Grammatik, die von indigenen Kreisen und sozialen Bewegungen bis zur Entstehung einer neuen sozial-ökologischen Agenda ausgearbeitet worden ist. Kurz gesagt: Der Übergang vom Washingtoner Konsens zum Rohstoffkonsens installierte Probleme und Paradoxien, die selbst den antagonistischen Charakter der sozialen Bewegungen und den Horizont des lateinamerikanischen kritischen Denkens neu konfigurierten und uns mit theoretischen und politischen Brüchen konfrontierten, die sich zu einem Bündel von ideologischen Positionen verdichteten, die nur schwer zu verarbeiten und zu lösen sind. Hinzu kommt, dass die gegenwärtige Phase der Verschärfung der extraktiven Dynamik mit ihren extremen Zahlen die Krise in ihren verschiedenen Auswirkungen verstärkt. Im Gegensatz zu früheren Perioden, in denen die Umwelt nicht nur die Konflikte betraf und selten explizit Gegenstand von Diskussionen war, weisen die ökoterritorialen Kämpfe in Lateinamerika heute auf eine Resignifikation des Problems in sozialer, territorialer, politischer und zivilisatorischer Hinsicht hin, die die hegemoniale Vision von Entwicklung und damit die Dynamik des neoliberalen Kapitalismus in Frage stellt.

Daher wird es notwendig, jene kollektiven Erfahrungen zu betrachten, die von Werten wie Gegenseitigkeit, Komplementarität, sozialer und ökologischer Gerechtigkeit, Fürsorge und Harmonie in den Inter- 
dependenzbeziehungen zwischen dem Menschlichen und dem Nichtmenschlichen genährt werden. Betrachtet man die Alternativen, so gibt es in Lateinamerika eine ökoterritoriale Perspektive, die zielgerichtet und mit Betonung auf die Agrarökologie vorgeht; eine indigene Perspektive mit gemeinschaftlicher Basis und mit Betonung auf der Dekolonialisierung und auf das buen vivir; eine ökofeministische Perspektive, mit Betonung auf der Care-Ethik und auf dem Ende des Patriarchats. Diese Herangehensweisen und Artikulationsweisen heben die Dekommodifizierung von Gemeingütern sowie die Notwendigkeit hervor, tragfähige Alternativvorschläge zu entwickeln, die unter anderem auf lokalen und regionalen Ökonomien, Erfahrungen in der Agrarökologie und (indigen-bäuerlichen) Gemeinschaftsräumen basieren.

Abschließend soll an eine kleine Tatsache erinnert werden: Wenn zu Beginn des Epochenwechsels die indigenen Völker mit der Infragestellung des Neoliberalismus, ihrem Protagonismus innerhalb der sozialen Kämpfe und der Ausarbeitung einer emanzipatorischen Sprache (vgl. buen vivir, Naturrechte, Autonomie, plurinationaler Staat) als wichtiger Akteur auftraten, so scheint das Ende des progressiven Zyklus und der Beginn einer neuen Epoche von den Kämpfen der Frauen geprägt zu sein. Dies wird in verschiedenen Maßstäben und Ebenen sichtbar, und zwar - wenn auch nicht ausschließlich - in den Widerständen gegen den Neo-Extraktivismus. Mit anderen Worten: Lateinamerika hat sich vom »Moment der Indigenen « zum »feministischen Moment « entwickelt. Diese Tendenz begleitet und ergänzt die Narrative des buen vivir und der Naturrechte, des ökofeministischen Diskurses zur Analogie von Territorium und Körper, der Care-Ethik und der Betonung der Interdependenz. So kommt zum Narrativ der Dekolonialisierung, das mit dem indigenen Moment zusammenhängt, nun die Forderung nach der Entpatriarchalisierung und nach einer ökologischen Dependenz hinzu, die mit dem feministischen Moment zusammenhängt.

Zusammenfassend gilt: In einem globalen ideologischen Kontext, in dem die unternehmerische und/oder fremdenfeindliche Rechte immer stärker dominiert, während sich die Linke in einem regionalen Krisenszenario befindet und sich teils gar den Konservativen zuwendet, stellt es eine große Herausforderung dar, kritisches antisystemisches Denken 
neu $\mathrm{zu}$ reflektieren und $\mathrm{zu}$ kreieren. In diesem Sinne sollte das Bauen von Brücken zwischen den verschiedenen auf dem Kontinent vorhandenen linken Lagern auf eine gemeinsame Diagnose der globalen Krise beruhen, die aus einer Logik der Aneignung und Ausbeutung der Natur hervorgeht und von einem neoliberalen Kapitalismus angetrieben wird, der eng mit dem Neo-Extraktivismus verknüpft ist. Ohne einen solchen Brückenschlag lässt sich der politische und intellektuelle Raum, den wir Linke nennen, nicht neu konfigurieren. Einfacher ausgedrückt, muss die Linke, um zu neuer Stärke zu finden, sowohl in Lateinamerika als auch in anderen Breitengraden nicht nur popular und pluralistisch sowie entschieden antipatriarchalisch agieren, sondern umfassend ökologisch. 


\section{Literatur}

Acosta, Alberto, La maldición de la abundancia, Quito 2009.

Acosta, Alberto, El Buen Vivir en el camino del post-desarrollo. Una lectura desde la Constitución de Montecristi, in: Friedrich Ebert Stiftung (Hg.), Policy Paper, núm. 9, 2010. URL: http://library.fes.de/pdffiles/bueros/quito/07671.pdf.

Acosta, Alberto, Extractivismo y neoextractivismo: dos caras de la misma maldición. (25.07.2012), URL: https://www.ecoportal.net/ temasespeciales/mineria/extractivismo_y_neoextractivismo_dos_ caras_de_la_misma_maldicion/: [letzter Zugriff: 09.02.2018].

Acosta, Alberto und Ulrich Brandt, Salidas del laberinto capitalista. Decrecimiento y Postextractivismo, Madrid 2017.

Acselard, Henri, Conflitos ambientais no Brasil, Río de Janeiro 2004.

Aguinaga, Margarita, Miriam Lang, Dunia Mokrani y Alejandra Santillana, Pensar desde el feminismo. Críticas y alternativas al desarrollo, in: Miriam Lang/Dunia Mokrani (Hg.), Más allá del desarrollo, Ecuador 2012, 55-82.

Antonelli, Mirta, Megaminería, desterritorialización del Estado y biopolítica, in: Astrolabio 7 (2011), 3-22. URL: https://revistas.unc. edu.ar/index.php/astrolabio/article/viewFile/592/3171.

Antonelli, Mirta, Megaminería Transnacional e invención de la cantera, in: Revista Nueva Sociedad (252/2014), 72-86. URL: http://nuso. org/articulo/megamineriatransnacional-e-invencion-del-mundocantera/.

Aranda, Darío, Lo primero es la desigualdad (16.01.2017), URL: https:// www.pagina12.com.ar/14484-lo- primero-es-la-desigualdad. 
Archilla, Mauricio (Hg.), Hasta cuando soñemos. Extractivismo e interculturalidad en el sur de la Guajira, Kolumbien 2015.

Auyero, Javier y María Fernanda Berti, La violencia en los márgenes, Buenos Aires 2013.

Baczko, Bronislaw, Les imaginaires sociaux, Paris 1984.

Bedoya, Jineth, Campamentos de explotación de niñas en zonas mineras alrededor de las minas hay redes organizadas de trata de mujeres (25.05.2013), URL: www.eltiempo.com/archivo/documento/CMS $-12824463$.

Bellamy, John, La Ecología de Marx: materialismo y naturaleza, Spanien 2000.

Bengoa, José, La emergencia indígena en América Latina, Santiago de Chile 2007.

Bertinat, Pablo, Otra energía es posible (29.07.2013), URL: www.enredando.org.ar/2013/07/29/no-podemos-discutir-politicasenergeticas-sin-discutir-el- modelo-de-desarrollo/.

Bertinat, Pablo, Eduardo d'Elia, Roberto Ochandio, Maristella Svampa und Enrique Viale, 20 mitos yrealidades del fracking, Buenos Aires 2017.

Bilder, Marisa, Las mujeres como sujetos políticos en las luchas contra la megaminería en Argentina. Registros acerca de la deconstrucción de dualismos en torno a la naturaleza y al género, Masterarbeit, Barcelona 2013.

Bolados, Paola und Alejandra Sánchez, Una ecología política feminista en construcción: El caso de las >mujeres de zonas de sacrificio en resistencia<, Región de Valparaíso, Chile, in: Psicoperspectivas 16 (2/2017), 33-42.

Bonneuil, Christophe und Jean-Baptiste Fressoz, L'événement antrhopocène. La terre, l'histoire et nous, Paris 2013.

Brandt, Ulrich und Markus Wissen, Crisis socioecológica y modo de vida imperial. Crisis y continuidad de las relaciones sociedadNaturaleza en el capitalismo, in: Carmen Ortiz/Sandra Ojeda (Hg.), Alternativas al capitalismo/colonialismo del siglo xxi, Ecuador 2013, 445470.

Bresser-Pereira, Luis Carlos, Globalización y competencia. Apuntes para una macroeconomía estructuralista del desarrollo, Buenos Aires 2010. 
Burchardt, Hans-Jürgen, El neo-extractivismo en el siglo xxi. Qué podemos aprender del ciclo de desarrollo más reciente en América Latina, in: Hans-Jürgen Burchardt/Rafael Domínguez/Carlos Larrea/Stefan Peters (Hg.), Nada dura para siempre. Neoextractivismo después del boom de las materias primas, Ecuador 2016, 55-89.

Calderón, Fernando und Mario Dos Santos, Sociedades sin atajos. Cultura, politica y reestructuración en América Latina, Buenos Aires 1995.

Canutto, Otaviano, The Commodity Super Cycle: Is This Time Different?, Economic Premise. The World Bank (150/2014), 1-3.

Carpio, Silvia, Integración energética sudamericana: entre la realidad, perspectivas e incertidumbres, in: Juan Carlos Guzmán Salinas (Hg.), Discursos y realidades. Matriz energética, políticas e integración. Plataforma Energética, Bolivien 2017, 91-138.

Castro, Gustavo, Efectos mundiales de las represas (2009), URL: www.eco-sitio.com.ar/node/266.

Cepal, El Estado frente a la autonomía de la mujeres, Santiago de Chile 2012. URL: http://repositorio.cepal.org/bitstream/handle/11362/ 27974/1/S1200259_es.pdf.

Cepal, Los bonos en la mira: aporte y carga para las mujeres. Observatorio de Igualdad de Género de América Latina y el Caribe, Santiago de Chile 2013. URL: www.cepal.org/publicaciones/xml/7/49307/20121042_OIG-ISSN_WEB.pdf.

Cepal, Anuario Estadístico de América Latina y el Caribe, Santiago de Chile 2015.

Chicaiza, Gloria, Mineras chinas en Ecuador: Nueva dependencia, Quito 2014.

Colectivo Voces de Alerta, 15 mitos y realidades sobre la minería transnacional en Argentina, Buenos Aires 2011.

Comini, Nicolás und Alejandro Frenkel, Una Unasur de baja intensidad. Modelos en pugna y desaceleración del proceso de integración en América del Sur, in: Revista Nueva Sociedad (250/2014), 58-77.

Composto, Claudia und Mina Lorena Navarro, Territorios en disputa: entre el despojo y las resistencias. La megaminería en México, in: Casa de Ondas (Hg.), Entender la descomposición, vislumbrar las posibilidades, Band 4, Mexiko 2011. 
Coraggio, José Luis, La presencia de economía social y solidaria (ess) y su institucionalización en América Latina. Estados Generales de la Economía Social y Solidaria, Ecuador 2011a. URL: www.coraggioeconomia.org/jlc_conferencias_conf.htm.

Coraggio, José Luis, Economía social y solidaria. El trabajo antes que el capital, Ecuador 2011b.

Coronil, Fernando, El Estado mágico. Naturaleza, dinero y modernidad en Venezuela, Venezuela 2002.

Danowski, Deborah und Eduardo Viveiros de Castro, Larret de monde, in: Emilie Haché (Hg.), De l'univers clos au monde infini, Paris 2014.

Daza Quintanilla, Mar, Nadia Ruiz Alba und Clara Ruiz Navarro, Pistas y aportes de los ecofeminismos en el Perú, in: Raphael Hoetmer/Miguel Castro/Mar Daza/José de Echave (Hg.), Minería y Movimientos sociales en el Perú. Instrumentos y propuestas para la defensa de la vida, el agua y los territorios, Lima 2013, 583-609.

De Echave, José, Alejandro Diez, Ludwig Huber, Bruno Revesz, Xavier Ricard Lanata und Martín Tanaka, Minería y Conflicto social, Lima 2009.

De Sousa Santos, Boaventura, Más allá de la gobernanza neoliberal: El Foro Social Mundial como legalidad y política cosmopolitas subalternas, in: Boaventura de Sousa Santos/César A. Rodríguez Garavito (Hg.), En El derecho y la globalización desde abajo. Hacia una legalidad cosmopolita, Mexiko 2007, 31-56.

De Sousa Santos, Boaventura, Epistemología del sur, Mexiko 2009.

Delgado, Gian Carlo, Ecología Política de la minería en América Latina. Aspectos socioeconómicos, legales y ambientales de la mega minería, Mexiko 2010

Delgado, Gian Carlo, Configuraciones del territorio: despojo, transiciones y alternativas, in: Mina Navarro/ Daniele Fini (Hg.), Despojo capitalista y luchas comunitarias en defensa de la vida en México, Claves desde la Ecología Política, Mexiko 2016, 51-70.

Descola, Philipe, Más allá de la naturaleza y la cultura, in: Leonardo Montenegro (Hg.), Cultura y Naturaleza. Aproximaciones a propósito del Bicentenario de Colombia, Bogotá 2011, 75-98. 
Di Chiro, Giovanna, La justicia social y la justicia ambiental en los Estados Unidos: La Naturaleza como comunidad, in: Michael Goldman (Hg.), Privatizing Nature. Political Struggles for the Global Commons, London 1998, 120-143. URL: www.scribd.com/doc/26939636/Ecologia-Politican\%C2\%BA-17-sept-1999.

Escobar, Arturo, El lugar de la naturaleza y la naturaleza del lugar: ¿globalización o postdesarrollo?, in: Edgardo Lander (Hg.), La colonialidad del saber. Eurocentrismo y ciencias sociales. Perspectivas latinoamericanas, Buenos Aires 2000, 68-77.

Escobar, Arturo, El post-desarrollo como concepto y práctica social, in: Daniel Mato (Hg.), Políticas de Economía, ambiente y sociedad en tiempos de globalización, Caracas 2005, 17-31.

Escobar, Arturo, Cultura y diferencia. La ontología política del campo de cultura y desarrollo, in: Walekeru Revista de investigación en Cultura y Desarrollo (2/2012). URL: http://biblioteca.hegoa.ehu.es/system/ ebooks/19420/original/Cultura_y_diferencia.pdf?1366975231.

Escobar, Arturo, Sentipensar con la tierra. Nueve lecturas sobre desarrollo, territorio y diferencia, Medellín 2014.

Esteva, Gustavo. 2007. "Commons: más allá de los conceptos de bien, derecho humano y propiedad . Entrevista con Gustavo Esteva sobre el abordaje y la gestión de los bienes comunes «. Entrevista realizada por Anne Becker en el marco de la Conferencia Internacional sobre Ciudadanía y Comunes, Mexiko, Dezember.

Federación Internacional por los Derechos Humanos (fidh). Criminalización de Defensores de Derechos humanos en el contexto de fenómenos industriales. Un fenómeno regional en América Latina . 2016. URL: https:// www.fidh.org/IMG/pdf/criminalisationobsangocto2015bassdef. pdf.

Feliz, Mariano, Proyecto sin clase: Crítica al neoestructuralismo como fundamento del neodesarrollismo, in: Mariano Feliz et al. (Hg.), Más allá del individuo. Clases sociales, transformaciones económicas y politicas estatales en la Argentina contemporánea, Buenos Aires 2012, 13-44.

Fondo de Acción Urgente-América Latina, Extractivismo en América Latina y su impacto en la vida de las mujeres, Bogotá 2017. 
Fontaine, Guillaume, Enfoques conceptuales y metodológicos para una sociología de los conflictos ambientales, escrito a propósito del petróleo y los grupos étnicos en la región amazónica (2003). URL: http://library.fes.de/pdf-files/bueros/kolumbien/01993/12.pdf. [letzter Zugriff: 03.02.2016].

Fundación Solón, Resultados de búsqueda para: El Bala (mehrere Veröffentlichungsdaten), URL: https://fundacionsolon.org/?s=El+Bala. [letzter Zugriff: 28.12.2016].

Gago, Verónica und Sandro Mezzadra, Para una crítica de las operaciones extractivas del capital, Patrón de acumulación y luchas sociales en el tiempo de la financiarización, in: Revista Nueva Sociedad (255/2015), 38-52

Galindo, María und Sonia Sánchez, Ninguna mujer nace para puta, Buenos Aires 2007.

Gandarillas, Marcos, Extractivismo y derechos laborales. Dilemas del caso boliviano, in: Juan Hernández Zubizarreta/Andrea Vicente/Koldo Irurzun (Hg.), Empresas transnacionales en América Latina: Análisis y propuestas del movimiento social y sindical, Spanien 2013, 218232. URL: www.cedib.org/wp- content/uploads/2013/07/empleo_hegoa_gandarillas.pdf.

Gandarillas, Marco, Bolivia: la década dorada del extractivismo, in: Marco Gandarillas (Hg.), Extractivismo: nuevos contextos de dominación y resistencia, Cochabamba 2014, 67-103.

García, Alan, El síndrome del perro del hortelano (28.10.2007), URL: https://elcomercio.pe/edicionimpresa/html/2007-10-28/el_ sindrome_del_perro_del_hort.html.

García, Álvaro, Geopolítica de la Amazonia, Bolivien 2012.

Gargallo, Francesca, Feminismos desde Abya Yala. Ideas y proposiciones de las Mujeres de 607 pueblos en nuestra América. Bogotá 2014.

Gaudichaud, Franck, >Progresismo transformista<, neoliberalismo maduro y resistencias sociales emergentes, in: Revista Osal XV (35/2014), 131-156. URL: www.rebelion.org/noticia.php?id=184776.

Giarracca, Norma und Miguel Teubal (Hg.), Actividades extractivas en expansión. ¿Reprimarización de la economía argentina?, Buenos Aires 2013. 
Gilligan, Carol, La ética del cuidado, Cuadernos de la Fundació Víctor Grífols i Lucas (30/2015). Barcelona 2015. URL: www.secpal.com/\%5CDocumentos \%5CBlog \%5Ccuaderno30.pdf.

Gilly, Adolfo, Chiapas, la razón ardiente, Mexiko 1997.

Goffman, Erving, Les cadres de l'experience, Paris 1991

Grupo de Estudios de Protesta Social y Acción Colectiva. 2006. Transformaciones de la protesta social en Argentina 1989-2003. Documento de Trabajo, Instituto G. Germani.

Gudynas, Eduardo, La ecología política del giro biocéntrico en la nueva Constitución del Ecuador, in: Revista de Estudios Sociales, 32 (2009a), 34-47.

Gudynas, Eduardo, Diez tesis urgentes sobre el nuevo extractivismo, in: Jürgen Schuldt/Alberto Acosta/Alberto Barandiarán/Anthony Bebbington/Mauricio Folchi/Alejandra Alayza/Eduardo Gudynas (Hg.), Extractivismo, Política y Sociedad, Quito 2009b.

Gudynas, Eduardo, Extractivismos. Ecología, economía y política de un modo de entender el desarrollo y la naturaleza, Bolivien 2015.

Gutiérrez, Raquel, Horizontes popular-comunitarios, Madrid 2017.

Haesbaert, Rogério, El mito de la desterritorialización. Del fin de los territorios a la multiterritorialidad, Mexiko 2011.

Hamilton, Clive, Les apprentis de sorciers. Raison et deraisons de la geo-ingenierie, Paris 2013.

Haraway, Donna, Antropoceno, Capitaloceno, Plantacionoceno, Chthuluceno: generando relaciones de parentesco, in: Revista Latinoamericana de Estudios Críticos Animales (2016).

Harvey, David, El nuevo imperialismo: Acumulación por desposesión, in: Socialist Register 2004, URL: bibliotecavirtual.clacso.org.ar/ar/libros/social/harvey.pdf.

Hoetmer, Raphael, Miguel Castro, Mar Daza, José de Echave, Minería y Movimientos sociales en el Perú. Instrumentos y propuestas para la defensa de la vida, el agua y los territorios, Lima 2013.

Jelin, Elizabeth, Los movimientos sociales en la Argentina contemporánea, in: Elizabeth Jelin (Hg.), Los nuevos movimientos sociales, Buenos Aires 1987, 13-40. 
Jelin, Elizabeth, La escala de los movimientos sociales, in: Elizabeth Jelin (Hg.), Más allá de la nación: las escalas múltiples de los movimientos sociales, Buenos Aires 2003.

Kaltmeier, Olaf, Refeudalisierung und Rechtsruck. Soziale Ungleichheit und politische Kultur in Lateinamerika. Bielefeld 2020.

Keinert, Fábio C., Os sentidos do lulismo: reforma gradual e pacto conservador, in: Tempo Social 24 (2/2012), 255-260, URL: www.scielo.br/pdf/ts/v24n2/v24n2a14.pdf.

Kessler, Gabriel, La sociedad Argentina hoy. Radiografia de una nueva estructura, Buenos Aires 2016.

Kolbert, Elizabeth, La sexta extinción, Barcelona 2015.

Korol, Claudia, Feminismos populares. Pedagogías y Políticas, Buenos Aires 2016.

Koselleck, Reinhart, Futuro pasado: para una semántica de los tiempos históricos, Barcelona 1993.

Laboratorio de Paz, Estado reconoce en cidh que no ha realizado estudio de impacto ambiental para Arco Minero, 2016, URL: www.laboratoriosdepaz.org/estado-reconoce-en-cidh-que-no-ha-realizadoestudio-de-impacto-ambiental-para-arco-minero/[letzter Zugriff: 18.12.2016].

Lander, Edgardo. Tensiones/contradicciones en torno al extractivismo en los procesos de cambio: Bolivia, Ecuador y Venezuela, in: Pablo Ospina/Edgardo Lander/Carlos Arze/Javier Gómez/Víctor Álvarez (Hg.), Promesas en su laberinto. Cambios y continuidades en los gobiernos progresistas de América Latina, Quito 2013.

Lander, Edgardo, El Neoextractivismo como modelo de desarrollo en América Latina y sus contradicciones, 2014, URL: https://mx.boell.org/sites/ default/files/edgardolander.pdf.

Lander, Edgardo und Santiago Arconada Rodríguez, Crisis civilizatioria. Experiencias de los gobiernos progresistas y debates em la izquierda latinoamericana, Bielefeld 2010.

Lang, Miriam und Dunia Mokrani, Más allá del desarrollo, Ecuador 2012. Leff, Enrique, La ecología política en América Latina: un campo de construcción, in: Héctor Alimonda (Hg.), Los tormentos de la materia. Aportes para una ecología política latinoamericana, Buenos Aires 2004. 
Leiras, Marcelo, Andrés Malamud und Pablo Stefanoni, Por qué retrocede la izquierda, Buenos Aires 2016.

Lemus, Jesús J., México a cielo abierto. De cómo el boom minero resquebrajó al país, Mexiko 2018.

León, Magdalena, Cambiar la economía para cambiar la vida. Desafíos de una economía para la vida, in: Alberto Acosta/Esperanza Martínez (Hg.), El buen vivir. Una vía para el desarrollo, Quito 2009.

López, Margarita, La protesta popular en la Venezuela contemporánea. Enfoque conceptual, metodológico y fuentes, in: J. Rodríguez (Hg.), Visiones del oficio. Historiadores venezolanos en el siglo xxi, Caracas 2012, 399-412.

Lowy, Michael, Ecosocialismo. La alternativa radical a la catástrofe ecológica capitalista, Buenos Aires 2011.

Machado Aráoz, Horacio, Naturaleza mineral. Una ecología politica del colonialismo moderno, Tesis doctoral, Universidad Nacional de Catamarca 2012.

Machado Aráoz, Horacio, Crisis ecológica, conflictos socioambientales y orden neocolonial. Las paradojas de Nuestra América en las fronteras del extractivismo, in: Revista Brasileira de Estudos Latino-Americanos 3 (1/2013), 118-155. URL: http://rebela.edugraf.ufsc.br/index. $\mathrm{php} / \mathrm{pc} / \mathrm{article} / \mathrm{view} / 137$.

Machado Aráoz, Horacio, Potosí, el origen, Buenos Aires 2014.

Machado, Decio und Raúl Zibechi, Cambiar el mundo desde arriba. Los límites del progresismo, Bogotá 2016.

Maldonado, Ángel. 2016. »Editorial«. Boletín Reinventerra.

Mançano, Bernardo, Movimentos socioterritoriais e movimentos socioespaciais. Contribuição teórica para uma leitura geográfica dos movimentos sociais, in: Revista Nera 6 (16/2005).

Mançano, Bernardo, Sobre la tipología de los territorios, 2008, URL: http:// web. ua.es/es/giecryal/documentos/documentos839/docs/bernardo-tipologia-de-territorios-espanol.pdf.

Marcos, Sylvia, Feminismos en el camino descolonial, in: Millán Márgara (Hg.), Más allá del feminismo. Caminos para andar, Red de feminismos descoloniales, 2014, 15-35. https://feminismosdescoloniales.files. wordpress.com/2015/08/macc81s-allacc81-con-porta.pdf. 
Martínez, Joan, El ecologismo de los pobres. Conflictos ambientales y lenguajes de valoración, Barcelona 2004.

Martínez, Joan, El triunfo del posextractivismo en 2015, in: Sinpermiso, 2015, URL: www.sinpermiso.info/textos/index.php?id=7778.

Max-Neef, Manfred, Antonio Elizalde und Martin Hopenhayn, Desarrollo a Escala Humana, una opción para el futuro, in: Development Dialogue (Sonderausgabe 1986).

Mealla, Eloy, El regreso del desarrollo, in: J. C. Scannone/D. García Delgado (Hg.), Ética, Desarrollo y Región, Buenos Aires 2006.

Melón, Daiana (Hg.), La Patria sojera. El modelo agrosojero en el Cono sur, Buenos Aires 2014.

Melucci, Alberto. 1991. »Quý a-t-il de nouveau dans les >nouveaux mouvements sociaux«". En La recomposition du politique, Louis Maheu y Antonio Sales, 129-162. Quebec: Presses de l'Université de Montréal.

Melucci, Alberto, Asumir un compromiso: identidad y movilización en los movimientos sociales, Zona-Abierta 69 (1994), 153-178.

Merlinsky, Gabriela (Hg.), Cartografias del conflicto ambiental en Argentina, Buenos Aires 2016.

Meyer, David und William Gamson, Marcos interpretativos de la oportunidad política, in: Doug McAdam/John McArthy/Mayer Zald (Hg.), Movimientos Sociales, perspectivas comparadas: oportunidades politicas, estructuras de movilización y marcos interpretativos culturales, Madrid 1999.

Millán, Márgara (Hg.), Más allá del feminismo. Caminos para andar, Red de feminismos descoloniales, 2013, URL: https://feminismosdescoloniales.files.wordpress.com/2015/08/macc81s-allacc81-con-porta.pdf.

Miranda, Boris, La rescalofrianter alianza entre la minería ilegal y la explotación sexual en Sudamérica, BBC Mundo 12.04. 2016, URL: www.bbc.com/mundo/noticias/2016/04/160406_america_latina_ alianza_siniestra_mineria_ilegal_trata_mujeres_prostitucion_sexual_bm [letzter Zugriff: 12.04.2016].

Modonesi, Massimo, Revoluciones pasivas en América Latina. Una aproximación gramsciana a la caracterización de los gobiernos pro- 
gresistas de inicio de siglo, in: Mabel Thwaites Rey (Hg.), El Estado en América Latina: continuidades y rupturas, Santiago de Chile 2010.

Modonesi, Massimo, Subalternización y revolución pasiva, in: El principio antagonista. Marxismo y acción política, Mexiko 2016.

Modonesi, Massimo und Maristella Svampa, Post-progresismo y horizontes emancipatorios en América Latina, 2016, URL: http:// rebelion.org/noticia.php?id=215469.

Moore, Jason W., El auge de la Ecología-Mundo Capitalista (I), Laberinto 38, 2013a, 9-26.

Moore, Jason W., El auge de la ecología-mundo capitalista (II): las fronteras mercantiles en el auge y decadencia de la apropiación máxima. Filosofia, política y economía en el Laberinto 39, 2013b, 21-30.

Moreno, Camila, La Economía Verde: una nueva fuente de acumulación primitiva, in: Alternativas al capitalismo/colonialismo del siglo xxi, Ecuador 2013.

Mujeres Creando, La virgen de los deseos, Buenos Aires 2005.

Murcia, Diana y Aura María Puyana, Mujeres indígenas y conflictos socioambientales, Bogotá 2016, URL: www.infoindigena.org/images/Publicaciones_generales/Genero/Mujeres-Indgenas-y-conflictossocio-ambientales-f.compressed.pdf.

Navarro, Mina L., Luchas por lo común. Antagonismo social contra el despojo capitalista de los bienes naturales en México, Mexiko 2015.

O'Connor, James, Causas naturales. Ensayo de marxismo ecológico, Buenos Aires 2001.

Ocmal, Cuando tiemblan los derechos. Extractivismo y Criminalización en América Latina, Quito 2011.

Oxfam, Las mujeres rurales de América Latina son luchadoras, no criminales, 2014, URL: https://www.oxfam.org/es/crece-peru-mexicoel-salvador-guatemala-bolivia/las-mujeres-rurales-de-americalatina-son-luchadoras [letzter Zugriff: 02.01.2017].

Oxfam, Desterrados, tierra, poder y desigualdad en América Latina, 2016, URL: https://www.oxfam.org/sites/www.oxfam.org/files/file_attachments/desterrados-full-es-29nov-web_o.pdf [letzter Zugriff: 17.01.2017]. 
Palacios, Mario, Vladimir Pinto und Raphael Hoetmer, Minería Transnacional, Comunidadesy las Luchas por el Territorio en el Perú: El caso de Conacami, Lima 2008.

Pardo, Daniel, Lo que se sabe de la supuesta masacre de 28 mineros en Venezuela, BBC Mundo 2016, URL: www.bbc.com/mundo/noticias/2016/03/160307_venezuela_mineros_tumeremo_dp [letzter $\mathrm{Zu}$ griff: 09.02.2018].

Paredes, Julieta, Hilando fino. Desde el feminismo comunitario, 2008, URL: http://mujeresdelmundobabel.org/files/2013/11/Julieta-ParedesHilando-Fino-desde-el-Fem- Comunitario.pdf.

Pascual, Marta und Yayo Herrero, Ecofeminismo, una propuesta para repensar el presente y construir el futuro, in: Boletín Ecologistas en Acción (10/2010).

Pérez, Amaia, Cadenas globales del cuidado, United Nations 2007, URL: http://mueveteporlaigualdad.org/publicaciones/cadenasglobalesdecuidado_orozco.pdf.

Pérez, Amaia, Prefacio, in: ONU Mujeres, En La economía feminista desde América Latina. Una hoja de ruta sobre los debates en la región, 2012, URL: www.unwomen.org/ /media/Headquarters/Media/Publications/es/Economiafeministad sdeamericalatina.pdf.

Peters, Stefan, Fin del ciclo: el neo-extractivismo en Suramérica frente a la caída de los precios de las materias primas. Un análisis desde una perspectiva de la teoría rentista, in: Has-Jürgen Burchardt/Rafael Domínguez/Carlos Larrea/Stefan Peters (Hg.), Nada dura para siempre. Neoextractivismo después del boom de las materias primas, Ecuador 2016, 21-54.

Pintos, Patricia, Cambios en la configuración de los territorios metropolitanos y proyectos en pugna en un país de la periferia capitalista, in: Patricia Pintos und Patricio Narodowski (Hg.), La privatopía sacrílega. Efectos del urbanismo privado en humedales de la cuenca baja del río lujan, Buenos Aires 2012.

Porto, Carlos, Geografías, Movimientos Sociales. Nuevas Territorialidades y Sustentabilidad, Mexiko 2001.

Porto, Carlos, Amazonia, Amazonias. Tensiones territoriales actuales in: Nueva Sociedad 272 (2017), 150-159. 
Preciado, Jaime, Paradigma social en debate; aportaciones del enfoque geopolítico crítico. La Celac en la integración autónoma de América Latina, in: Martha N. Ruiz (Hg.), América Latina la crisis global, problemas y desafios, Argentinien 2014.

Puleo, Alicia, Ecofeminismo para otro mundo posible, 2011, URL: www.mujeresenred.net/spip.php?article1921.

Ramírez, Franklin, Decisionismos transformacionales, conflicto político y vínculo plebeyo. La gestión del poder en el nuevo progresismo sudamericano. El Estado en América Latina, 2008.

Ramírez García, Hugo Saúl, Biotecnología y Ecofeminismo. Un estudio de contexto, riesgos y alternativas, Mexiko 2012.

Red de Feminismos Descoloniales, ¿Quiénes somos?, URL: https:// feminismosdescoloniales.wordpress.com/about/[letzter Zugriff: 31.12.2016].

Resumen Latinoamericano. 2015. »Entrevista a Magdalena León, economista feminista miembro de la Red Latinoamericana de Mujeres >Auditar la deuda sirve para hacer reivindicaciones con conocimiento; no pagar es muy simplista«". Resumen Latinoamericano. www.resumenlatinoamericano.org/2015/01/08/entrevistaamagdalena-leon-economista-feminista-miembro-de-la-redlatinoamericana-de-mujeresauditar-la-deuda-sirve-para-hacerreivindicaciones-con-conocimiento-no-pagar-es-muy-simplista/.

Rivas, Antonio, El análisis de marcos: Una metodología para el estudio de los movimientos sociales, in: P. Ibarra und B. Tejerina (Hg.), Los movimientos sociales. Transformaciones politicas y cambios culturales, Madrid: 1998.

Roa, Tatiana und Hernán Scandizzo (Hg.), Qué entendemos por energía extrema, in: Extremas. Nuevas fronteras del extractivismo energético en Latinoamérica, Kolumbien 2017.

Roa, Tatiana und Luisa María Navas (Hg.), Extractivismo, conflictos y resistencias, Kolumbien 2014.

Roa, Tatiana, María Cecilia Roa García, Jessica Toloza Chaparro und Luisa María Navas Camacho, Como el agua y el aceite. Conflictos socioambientales por la extracción petrolera, Bogotá 2017. 
Rodríguez, Corina, Economía feminista y economía del cuidado. Aportes conceptuales para el estudio de la desigualdad, in: Revista Nueva Sociedad 256 (2015).

Rodríguez Garavito, César El derecho en los campos minados, in: Etnicidad.gov: los recursos naturales, los pueblos indigenas y el derecho a la consulta previa en los campos sociales minados, Bogotá 2012, 8- 24.

Rodríguez Garavito, César (Hg.), Extractivismo versus derechos humanos. Crónicas de los nuevos campos minados en el Sur Global, Buenos Aires 2016.

Romero, César und Francisco Ruiz, Dinámica de la minería a pequeña escala como sistema emergente, in: K. Gabbert und Alexandra Martínez (Hg.), Venezuela desde adentro. Ocho investigaciones para un debate necesario, Quito 2018, 87-144.

Sacher, William, Segunda contradicción del capitalismo y megaminería. Reflexiones teóricas y empíricas a partir del caso argentino, Tesis doctoral, Ecuador 2016.

Sack, Robert, Human territoriality: Its Theory and history, Cambridge 1986. Saguier, Marcelo und Guillermo Peinado, Minería transnacional y desarrollo en el kirchnerismo, in: Flacso-Isa Joint International Conferencie Global and Regional Powers in a Changing World, Buenos Aires 2014.

Salama, Pierre, China-Brasil: industrialización y sdesindustrialización temprana, in: Open Journal Sistem (2011), URL: www.revistas.unal.edu.co/index.php/ceconomia/article/view/35841/39710.

Salama, Pierre, Les économies emergentes latino-américaines. Entre cigales et fourmis, Paris 2012.

Salama, Pierre, ¿Se redujo la desigualdad en América Latina? Notas sobre una ilusión, in: Nueva sociedad (2015).

Santos, M. 2005. »O retorno do territorio«. Reforma agraria y lucha por la tierra en América Latina, territorios y movimientos sociales vi, núm. 16.

Sassen, Saskia, Los espectros de la globalización, Buenos Aires 2003 a.

Sassen, Saskia, Contrageografias de la globalización. Género y ciudadanía en los circuitos fronterizos, Madrid 2003b.

Sassen, Saskia, Expulsiones, Brutalidad y complejidad en la economía global, Buenos Aires 2015. 
Schavelzon, Salvador, El Estado neoliberal terminó gobernando el progresismo 2016, URL: http://anarquiacoronada.blogspot.com.ar/2016/05/ el-estado-neoliberal-termino- gobernando.html [letzter Zugriff: 12.05.2016].

Schuldt, Jürgen und Alberto Acosta, Petróleo, rentismo y subdesarrollo. ¿Una maldición sin solución?, in: Jürgen Schuldt/Alberto Acosta/Alberto Barandiarán/Anthony Bebbington/Mauricio Folchi/Alejandra Alayza/Eduardo Gudynas (Hg.), Extractivismo, Politica y Sociedad, Quito 2009.

Sempere, Joaquim, Sobre la revolución Rusa y el comunismo del siglo $x x, 2015$, URL: https://centenarirevoluciorussa.wordpress.com/ 2015/05/01/31/.

Shiva, Vandana und María Mies, La práxis del ecofeminismo. Biotecnología, consumo, reproducción, Icaria Editorial, Barcelona 1998.

Singer, André, Os sentidos do lulismo: reforma gradual e pacto conservador, in: Tempo Social 24 (2/2012), URL: www.scielo.br/pdf/ts/v24n2/v24n2a14.pdf.

"Situación de los derechos humanos de los pueblos indígenas en Bolivia«. 2010. Documento de las Organizaciones de Derechos Humanos para el Foro Permanente para los Pueblos Indígenas, La Paz, Bolivia.

Slipak, Ariel, La expansión de China en América Latina: incidencia en los vínculos comerciales argentino- brasileros, in: Congreso de Economía Política Internacional (2014), Universidad Nacional de Moreno, Buenos Aires.

Sola, María und Lorena Bottaro, La expansión del extractivismo y los conflictos socioambientales en torno a la megaminería a cielo abierto en Argentina, in: Revista latinoamericana Pacarina de Ciencias Sociales y Humanidades 4 (2013), 89-100.

Solón, Pablo (Hg.), Alternativas Sistémicas, La Paz 2017.

Subirats, Joan, Algunos apuntes sobre la relación entre los bienes comunes y la economía social y solidaria, in: Otra Economía 5 (9/2011), 195-204.

Svampa, Maristella, Cambio de Época. Movimientos sociales y poder político, Buenos Aires 2008. 
Svampa, Maristella, Movimientos Sociales, matrices socio-políticos y nuevos escenarios en América Latina, Deutschland 2010.

Svampa, Maristella, Consenso de los Commodities y lenguajes de valoración en América Latina, in: Nueva Sociedad (2013), URL: http:// nuso.org/articulo/consenso-de-los-commodities-y-lenguajes-devaloracion-en-america-latina/.

Svampa, Maristella, Feminismos del sur y ecofeminismos, in: Nueva Sociedad 256 (2015), URL: http://nuso.org/media/articles/ downloads/_1.pdf.

Svampa, Maristella, Debates Latinoamericanos. Indianismo, Desarrollo, Dependencia y Populismo, Buenos Aires 2016.

Svampa, Maristella, Del cambio de época al fin de ciclo. Gobiernos Progresistas, extractivismo y movimientos sociales, Buenos Aires 2017.

Svampa, Maristella und Ariel Slipak, China en América Latina: Del Consenso de los Commodities al Consenso de Beijing, Ensambles 2016.

Svampa, Maristella und Enrique Viale, Maldesarrollo. La Argentina del extractivismo y el despojo, Buenos Aires 2014.

Svampa, Maristella und Enrique Viale, La trumpización de la política ambiental, in: Clarín (2017), URL: https://www.clarin.com/opinion/ trumpizacion-politica-ambiental_o_HkCTc9bae.html.

Tarrow, Sidney, El poder en movimiento. Los movimientos sociales, la acción colectiva y la política, Madrid 1997.

Terán, Emiliano, Las nuevas fronteras de las commodities en Venezuela: extractivismo, crisis histórica y disputas territoriales, in: Ciencia Política 11 (21/2016), 251-285.

Tilly, Charles und Sidney Tarrow, La politica del conflitto, Mailand 2008.

Toledo, Víctor, Salir del capitalismo. La revolución agroecológica y la economía social y solidaria en América Latina, in: J. Luis Coraggio (Hg.), Economía social y solidaria en movimiento, Buenos Aires 2016, 143-158.

Touraine, Alain, Actores sociales y sistema político en América Latina, Santiago 1988.

Unceta, Koldo, Desarrollo, subdesarrollo, maldesarrollo y postdesarrollo. Una mirada Transdisciplinar sobre el debate y sus implicacio- 
nes, in: Carta Latinoamericana, Contribuciones en Desarrollo y Sociedad en América Latina (7/2009).

Unceta, Koldo, Más allá del crecimiento. Debates sobre Desarrollo y Posdesarrollo, Buenos Aires 2015.

Valenzuela, José Manuel, Trazos de sangre y fuego. Bionecropolítica y juvenicidio en América Latina, Bielefeld, 2018.

Villegas, Pablo N., Notas sobre movimientos sociales y gobiernos progresistas, in: Extractivismo: nuevos contextos de dominación y resistencias, Cochabamba 2014, 9-66.

Vittor, Luis, Conacami: 10 años tejiendo resistencias a la minería en Perú. América Latina en movimiento, 2009, URL: http://alainet.org/active/30470.

Viveiros de Castro, Eduardo, El cascabel del Chamán es un acelerador de partículas. En La mirada del jaguar. Introducción al perspectivismo amerindio (Interviews), 9-34, Buenos Aires 2008.

Wainer, Andrés/Martín Schorr, Concentración y extranjerización del capital en la Argentina Reciente: ¿Mayor autonomía nacional o incremento de la dependencia?, in: Latin American Research Review 49 (3/2014), 103-125.

Zavaletta, René, Lo nacional-popular en Bolivia, La Paz 2009.

Zibechi, Raúl, Tensiones entre extractivismo y redistribución en los procesos de cambio. Alternativas al desarrollo extractivista $y$ antropocéntrico, 2011, URL: www.aldeah.org/es/raul-zibechi-tensionesentre-extractivismo-yredistribucion-en-los-procesos-de-cambiode-america-lat.

Zibechi, Raúl, El fin del consenso lulista, 2013, URL: http://asociaciondeusuarios.blogspot.com.ar/2013/07/el-fin-del-consenso-lulista.html [letzter Zugriff: 28.12.2016].

Zibechi, Raúl, El mito del progresismo y la desigualdad en América Latina, 2015, URL: www.urngmaiz.org.gt/2015/11/el-mito-delprogresismo-y-la-desigualdad-en-america-latina/ [letzter Zugriff: 28.12.2016].

Zorrilla, Carlos, William Sacher und Alberto Acosta, 21 preguntas para entender la minería del siglo 21 (2012), URL: http://rebelion.org/docs/ 138009.pdf. 


